\title{
Nonexistence of wandering domains for strongly dissipative infinitely renormalizable Hénon maps at the boundary of chaos
}

\author{
Dyi-Shing $\mathrm{Ou}^{1,2}$
}

Received: 29 December 2016 / Accepted: 10 July 2019 / Published online: 23 July 2019 (C) The Author(s) 2019

\begin{abstract}
This article generalizes the nonexistence of wandering domains from unimodal maps to infinitely period-doubling renormalizable Hénon-like maps in the strongly dissipative (area contracting) regime. This solves an open problem proposed by van Strien (Discrete Contin Dyn Syst 27(2):557-588, 2010) and Lyubich and Martens (Invent Math 186(1):115-189, 2011). We partition the phase space of a Hénon-like map into two regions: the good region and the bad region. The good region is where the method of proof for unimodal maps applies to Hénon-like maps, while the bad region is where serious difficulties occur. These difficulties are resolved by the Two-Row Lemma, an inequality that relates the contraction of areas to the contraction of bad regions. After analyzing the competition of the two types of contraction, we show that the case of bad regions happens at most finitely many times and complete the proof. As an application, the theorem enriches our understanding of the topological structure of the heteroclinic web: the union of the stable manifolds of periodic orbits forms a dense set in the domain.
\end{abstract}

The first version of the manuscript was done at Stony Brook University as a part of the author's $\mathrm{PhD}$ thesis. The final revision was completed at IMPAN.

$\bowtie$ Dyi-Shing Ou

dsou@impan.pl

1 Department of Mathematics, Stony Brook University, Stony Brook, NY 11794-3651, USA

2 Present Address: Institute of Mathematics, Polish Academy of Sciences, ul. Śniadeckich 8, 00-656 Warszawa, Poland 
Mathematics Subject Classification 37E30 $\cdot 37$ C70 $~ 37 E 20 \cdot 37 D 45$

\section{List of symbols}

$A, B, C \quad$ Partition of the domain $D$ for a unimodal map, Definition 3.1

$A, B, C$ Partition of the domain $D$ for a Hénon-like map, Definition 4.10

$c^{(n)} \quad$ Critical orbit, Sect. 3

$C_{n}(j) \quad$ Rescaling level $j$, Definition 5.1

$C_{n}^{l}(j) \quad$ Left component of $C_{n}(j)$, Proposition 5.2

$C_{n}^{r}(j) \quad$ Right component of $C_{n}(j)$, Proposition 5.2

$D \quad$ The maximal invariant set in $I^{h} \times I^{v}$, Definition 4.6

$\epsilon \quad$ Perturbation component for a Hénon-like map, Sect. 4.1

$f \quad$ Unimodal component for a Hénon-like map, Sect. 4.1

$F \quad$ Hénon-like map, Sect. 4.1

$g \quad$ Fixed point of the unimodal renormalization, Proposition 3.5

$G \quad$ Fixed point of the Hénon renormalization, Sect. 4.3

$h \quad x$-component of a Hénon-like map, Sect. 4.1

$H \quad$ Nonlinear part of the Hénon rescaling, Sect. 4.3

$\mathcal{H} \quad$ Class of Hénon-like maps, Sect. 4.1

. $\quad$ Order reversing involution, Sect. 3

$I(\epsilon) \quad$ Complex $\epsilon$-neighborhood of the interval $I$, Sect. 2

$I^{h} \quad$ Horizontal domain for a Hénon-like map, Sect. 4.1

$I^{v} \quad$ Vertical domain for a Hénon-like map, Sect. 4.1

$\mathcal{I} \quad$ Class of infinitely renormalizable Hénon-like maps, Sect. 4.3

$J_{n} \quad J$-closest approach, Definition 6.5

$K_{n} \quad$ Boundary between the regions, Definition 8.1

$l \quad$ Horizontal size, Definition 6.7

$\Lambda \quad$ Affine part of the Hénon rescaling, Proposition 4.14

$\lambda \quad$ The rescaling constant of g, Proposition 3.5

$\lambda_{n} \quad\left|s_{n}^{\prime}\right|$, Sect. 4.3

$n^{(j)} \quad$ Time span in good regions, Definition 10.7

$p_{n}(j) \quad$ Periodic point of $F_{n}$ with period $2^{j}$, Definition 5.1

$\phi \quad$ Hénon rescaling, Proposition 4.14

$\Phi_{n}^{j} \quad$ Nonlinear rescaling from renormalization scale $n$ to $n+j$, Definition 5.1

$r(n) \quad$ Renormalization scale of $J_{n}$, Definition 6.5

$R \quad$ Unimodal renormalization, Definition 3.4

$R \quad$ Hénon renormalization, Proposition 4.14

$s \quad$ Affine part of the Hénon rescaling, Proposition 4.14

$s \quad$ Rescaling of the unimodal renormalization, Definition 3.4

$S \quad$ Schwarzian derivative, Sect. 2 
$\tau$

$\mathcal{U}$

$\mathcal{U}^{r}$

$\mathcal{U}_{\delta}$

$v$

$w$

$W^{t}(-1)$

$W^{t}(0)$

$W_{n}^{t}(j)$

The tip of an infinitely renormalizable Hénon-like map, Definition 5.3

Class of unimodal maps, Sect. 3

Class of renormalizable unimodal maps, Definition 3.2

Class of unimodal maps with holomorphic extension on a $\delta$ neighborhood, Sect. 4.1

Vertical size, Definition 6.7

Thickness, Definition 10.1

Local stable manifold of $p(-1)$, Definition 4.6

Local stable manifold of $p(0)$, Definition 4.8

Local stable manifold of $p_{n}(j)$, Definition 5.1

\section{Introduction}

This article studies the existence of wandering domains for Hénon-like maps. These maps are real two-dimensional continuous maps of the form

$$
F(x, y)=(f(x)-\epsilon(x, y), x)
$$

where $f$ is a unimodal map and $\epsilon$ is a small perturbation. The maps are a generalization of classical Hénon maps [36] (a two-parameter family of polynomial maps) to the analytic settings and an extension of unimodal maps to higher dimensions. For renormalization purposes, the maps under consideration are all real analytic and strongly dissipative (the Jacobian $\left|\frac{\partial \epsilon}{\partial y}\right|$ is small ${ }^{1}$ ).

Strongly dissipative Hénon-like maps are the maps that are closest to unimodal maps, with which they share many dynamical properties. For example, the unimodal renormalization can be adapted to Hénon-like maps $[11,34,48]$, the renormalization operator is hyperbolic [11, Theorem 4.1], and an infinitely renormalizable Hénon-like map has an attracting Cantor set [11, Subsection 5.2]. However, there are also some properties that make Hénon-like maps distinct from unimodal maps. For example, the Cantor set for infinitely renormalizable Hénon-like maps is not rigid [11, Theorem 10.1] and there is no finite-dimensional family of Hénon-like maps which serves as a universal model [35]. In the degenerate case, unimodal maps do not have wandering intervals $[5,15,16,30,47,52]$. It is natural to ask whether this property can be extended to Hénon-like maps.

The study of wandering domains has a broad interest in dynamics. In dimension one, the problem has been widely studied in many different contexts. However, so far only a few types of systems in higher dimensions were found not to have wandering domains.

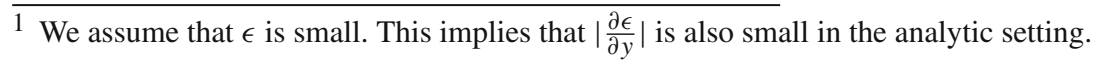


In one real dimension, a wandering interval $J$ of a map $f$ is a nonempty open interval such that its forward iterates $J, f(J), f^{2}(J), \ldots$ are disjoint and do not approach a periodic orbit. Showing the absence of wandering intervals in a system is important to solve the classification problem. For circle homeomorphisms, a classical theorem of Poincaré [61] states that an orientation-preserving circle homeomorphism with irrational rotation number (the average rotation of an orbit) and no wandering interval is conjugate to the rigid rotation with the same rotation number. The work of Denjoy [18] and other subsequent papers $[38,59,63,68]$ showed that an orientation-preserving circle homeomorphism with irrational rotation number does not have a wandering domain if the map is smooth enough. Counterexamples [18,31,37] indicated that the regularity conditions are essential. For unimodal maps, Guckenheimer [30] developed an analogue of the Poincaré theory by showing that if two unimodal maps have the same kneading sequence [55] which is not periodic and neither map has a wandering interval, then the two maps are topologically conjugate. In particular, he showed that $C^{3}$ unimodal maps with negative Schwarzian derivative do not have wandering intervals. Some other papers relaxed the hypothesis or generalized the theorem to multimodal maps $[5,15,16,47,52]$. Similar to the case of circle homeomorphisms, the papers all assume some nonflatness control around the critical point because of the counterexamples in $[13,64]$.

In one complex dimension, a wandering domain is a Fatou component that has a disjoint orbit. Sullivan's no-wandering-domain theorem [65] says that a rational map on the Riemann sphere does not have a wandering domain. As a consequence, it completes the last piece of the classification of Fatou components [10,23-25]. Sullivan's Theorem was extended to entire mappings with finitely many singular values $[22,29]$. However, in general, a transcendental entire function can have either an escaping [3] or an oscillating wandering domain [21].

In higher real dimensions, the problem of the existence of wandering domains is still wide open. There is no reason to expect the absence of wandering domains [67], especially when the regularity is not enough as suggested by the one dimensional systems $[6,53,54]$. The classification problem breaks down between any two different levels of differentiability for diffeomorphisms on $d$-manifold with $d \neq 1,4[32,33]$. Examples of real polynomial skewproduct maps have been found having wandering domains [2]. Nonhyperbolic phenomena also play a role in building counterexamples $[9,42,44]$. A relevant work by Kiriki and Soma [42] found Hénon-like maps having wandering domains by using a homoclinic tangency of some saddle fixed point $[41,43]$. On the other hand, there are studies $[45,46,57,60]$ suggesting that some types of systems may have no wandering domains. However, this has been proved in just some such systems $[6,58]$. 
In higher complex dimensions, examples of transcendental maps with wandering domains can be constructed from one-dimensional examples [26] by taking direct products. For polynomial maps, very little was known until recent developments on polynomial skew-products. In contrast to the complex one-dimensional case, Astorg et al. [2] found a polynomial skew-product possessing a wandering Fatou component. The reader can refer to the survey [62] for more details about other relevant works. For complex Hénon maps, ${ }^{2}$ a recent paper by Arosio et al. [1] found a transcendental Hénon map exhibiting a wandering domain. Nevertheless, the problem is still unsolved [4] for complex polynomial Hénon maps.

In this paper, a wandering domain is a nonempty open set that is disjoint from the stable manifolds of all saddle periodic points. This definition is equivalent to the classical notion of wandering intervals in the unimodal setting (see Remark 6.2). Our definition allows us to study topological properties of the heteroclinic web by using wandering domains (Corollary 10.16).

Our main theorem (Theorem 10.14) can be formulated as follows.

Theorem A strongly dissipative infinitely period-doubling renormalizable Hénon-like map does not have wandering domains.

The theorem covers maps in the higher-dimensional nonhyperbolic setting [11, Theorem 6.3] and solves an open problem proposed by van Strien [48] and Lyubich and Martens [48]. Here, we focus on infinitely renormalizable maps of period-doubling combinatorics type. This is one of the most fundamental types of maps because the maps are located on the boundary of chaos in the onedimensional case [49]. Several papers $[6,50,51]$ in different contexts show that the "infinitely renormalizable" condition is sufficient for proving the absence of wandering domains.

The theorem is compatible with a relevant work by Kiriki and Soma [42]. In our article, the Hénon-like maps under consideration are real analytic, and the fixed points are far from having a homoclinic tangency. However, in their article, the maps they found with wandering domains have only finite differentiability, and their construction relies on the existence of a homoclinic tangency of a fixed point $[41,43]$.

The theorem also enriches our understanding of the topological structure of the attracting sets. An attracting set is a decreasing intersection $\bigcap_{n=1}^{\infty} f^{n}(A)$ where $A$ is a compact invariant set. Hénon maps are famous for their chaotic limiting behavior since Hénon first discovered the strange attractors in the classical Hénon family [36]. The $\omega$-limit set of a point $x$ is $\lim _{\sup _{n \rightarrow \infty}}\left\{f^{n}(x)\right\}$. It is a kind of attracting set. For an infinitely period-doubling renormalizable

\footnotetext{
2 The study of complex Hénon maps is inspired by the classification of polynomial automorphisms [27]. The maps $f$ as in (1.1) cover a broader class of functions containing arbitrary polynomials [39,40] or analytic maps [19].
} 
Hénon-like map, $\omega$-limit sets are classified into two categories [28,48]: a saddle periodic orbit or the renormalization Cantor set that is conjugate to the dyadic adding machine. From this dichotomy, a wandering domain is equivalently a nonempty open subset of the basin of the Cantor attractor. As a consequence of the main theorem, we have the following.

Corollary The union of the stable manifolds is dense.

In other words, the basin of the Cantor set has no interior even though it has full Lebesgue measure.

To prove the theorem, we partition the phase space of a Hénon-like map into two regions, the good region and the bad region, according to how similar the map is to a unimodal map. In the good region, we show that an expansion estimate from unimodal maps can be promoted to Hénon-like maps. In the bad region, nonhyperbolic phenomena occur and the expansion estimate breaks down. In this paper, we introduce the Two-Row Lemma (Lemma 10.11) to relate the contraction of areas (thickness) to the contraction of bad regions. By applying the lemma inductively, we show that the number of times that the obstacle occurs is finite and hence the expansion estimate applies to Hénon-like maps with finitely many exceptions.

The methods developed in this paper may also be applied to other systems in order to generalize dynamical properties from lower-dimensional to higherdimensional systems. One possibility is to extend the theorem to infinitely renormalizable Hénon-like maps with arbitrary combinatorics [34]. The definition of the bad region carries over to the arbitrary combinatorics case. It is also possible to generalize the estimate for the contraction of area because these maps have a universal shape around the tip [34, Theorem 6.1].

\section{Outline of the article}

Sections 2, 3, 4, and 5 are the preliminaries to the proof of the theorem. Most of the theorems in Sects. 4 and 5.1 can be found in [11,48].

The proof of the nonexistence of wandering domains is motivated by the proof in the degenerate case. The fact that a Hénon-like map is degenerate means that $\epsilon=0$ in (1.1). In this case, the dynamics of the map degenerates to the unimodal dynamics. In Sect. 7, a short proof of the nonexistence of wandering intervals for infinitely renormalizable unimodal maps is presented by identifying unimodal maps with degenerate Hénon-like maps. The proof is by contradiction, i.e. we assume that there exists a wandering interval $J$. We apply the Hénon renormalization instead of the standard unimodal renormalization to study the dynamics of the rescaled orbit of $J$ that approaches the critical value in the closest possible way. The rescaled orbit is called the $J$-closest approach (Definition 6.1). The proof argues that the lengths of the 
elements in the orbit expand at a definite rate. Hence, the lengths go to infinity, which leads to a contradiction. The expansion argument inspires the proof for the Hénon case.

The proof of the main theorem, given in Sects. 6, 8, 9, and 10, is sketched here. Suppose that a Hénon-like map has a wandering domain $J$. In Sect. 6, we define the $J$-closest approach $\left\{J_{n}\right\}_{n \geq 0}$ for Hénon-like maps. Each element $J_{n}$ belongs to some appropriate renormalization scale (the domain of some renormalization $R^{r(n)} F$ ). The transition between two consecutive orbit elements $J_{n} \rightarrow J_{n+1}$ is called one step. Inspired by the expansion estimate from the degenerate case, we study the expansion rate of the horizontal sizes $l_{n}$ of the elements. The horizontal size of a set is the length of its projection to the first coordinate (Definition 6.7). Our aim is to show that the horizontal sizes of the orbit elements go to infinity.

In the degenerate case, the expansion estimate says that the horizontal sizes expand at a uniform rate, and hence the sizes go to infinity. Unfortunately, the estimate does not hold in the nondegenerate case. There are two aspects that make the proof for the nondegenerate case different:

1. The existence of bad regions.

2. Thickness.

The good region and the bad region, introduced in Sect. 8, divide the phase space into two regions depending on the extent to which the map behaves like a unimodal map. Each renormalization scale (the domain of some renormalization $R^{n} F$ ) has its own good region and bad region, and the sizes of the bad regions contract super-exponentially as the number of renormalizations increases ([11, Theorem 4.1] and Definition 8.1).

When the elements in a closest approach stay in the good regions, the Hénon-like map behaves like a unimodal map. In particular, we show that the expansion estimate can be generalized to Hénon-like maps in Sect. 9.

However, when an element $J_{n}$ enters the bad region of some scale, the expansion estimate breaks down and the horizontal size contracts. To estimate the rate of contraction, we introduce a quantity called "thickness" (Definition 10.1). The thickness $w_{n+1}$ of the next element $J_{n+1}$ offers a good approximation for the horizontal size $l_{n+1}$ of the element. We will show that the contraction rate of the thicknesses has the same size as the Jacobian of the map (Proposition 10.5). For a strongly dissipative Hénon-like map, the Jacobian is small and hence the contraction is substantial. The contraction produces the main obstruction to showing that the horizontal sizes go to infinity.

The key observation is that a closest approach can have at most finitely many entries to bad regions (Proposition 10.13). When an element $J_{n}$ enters the bad region, the horizontal size becomes smaller but the size of the bad region also contracts because the element is rescaled many times (Proposition 10.10). Roughly speaking, we prove that the sizes of bad regions contract 
faster than the horizontal sizes. Hence, the elements cannot enter bad regions infinitely many times. The actual proof is quite delicate because the contraction of horizontal sizes also depends on the time span in good regions (Definition 10.7). The Two-Row Lemma (Lemma 10.11) relates the contraction of thicknesses, the expansion of horizontal sizes in good regions, the time span in good regions, and the size of a bad region when the closest approach enters bad regions twice. Finally, the key observation is proved by applying the Two-Row Lemma inductively (Lemma 10.12).

In summary, we may assume without lose of generality that a closest approach stays in the good region forever by starting from the tail of a rescaled orbit. However, the horizontal sizes expand in the good regions and go to infinity, which leads to a contradiction. Therefore, wandering domains cannot exist.

\section{Notations and preliminaries}

Assume that $f$ is a real- or complex-valued function defined on a set $S$. The sup norm of $f$ on $S$ is denoted by $\|f\|_{S}$. The subscript is omitted whenever the context is clear.

Let $I$ be an interval on the real line and $\delta>0$. The length of $I$ is denoted by $|I|$. The complex $\delta$-neighborhood of $I$ is the set $I(\delta)=$ $\left\{z \in \mathbb{C} ;\left|z-z^{\prime}\right|<\delta\right.$ for some $\left.z^{\prime} \in I\right\}$. As an application of the Cauchy integral formula, for each integer $n \geq 1$, there exists a constant $c>0$ such that

$$
\left\|f^{(n)}\right\|_{I} \leq c\|f\|_{I(\delta)}
$$

for all holomorphic maps $f$ on $I(\delta)$ where $f^{(n)}$ is the $n$-th derivative of $f$. A similar estimate holds for the partial derivatives of multi-variable holomorphic maps.

Assume that $f$ is a $C^{3}$ real-valued function on an interval. The Schwarzian derivative of $f$ is

$$
(S f)(x)=\left(\frac{f^{\prime \prime}(x)}{f^{\prime}(x)}\right)^{\prime}-\frac{1}{2}\left(\frac{f^{\prime \prime}(x)}{f^{\prime}(x)}\right)^{2}=\frac{f^{\prime \prime \prime}(x)}{f^{\prime}(x)}-\frac{3}{2}\left(\frac{f^{\prime \prime}(x)}{f^{\prime}(x)}\right)^{2}
$$

whenever $f^{\prime}(x) \neq 0$. The map $f$ is said to have negative Schwarzian derivative if $S f(x)<0$ for all $x \in I$ with $f^{\prime}(x) \neq 0$.

The projections $\pi_{x}$ and $\pi_{y}$ are the maps $\pi_{x}(x, y)=x$ and $\pi_{y}(x, y)=y$.

\section{Unimodal maps}

In this section, we give a short review of the procedure of unimodal renormalization. The goal is to introduce the hyperbolic fixed point of the 
Fig. 1 The partition $\{A, B, C\}$ for a unimodal map. The parabola is the graph of a unimodal map. The points $p(0), p^{(1)}$, and $p^{(2)}$ are defined in Definition 3.1

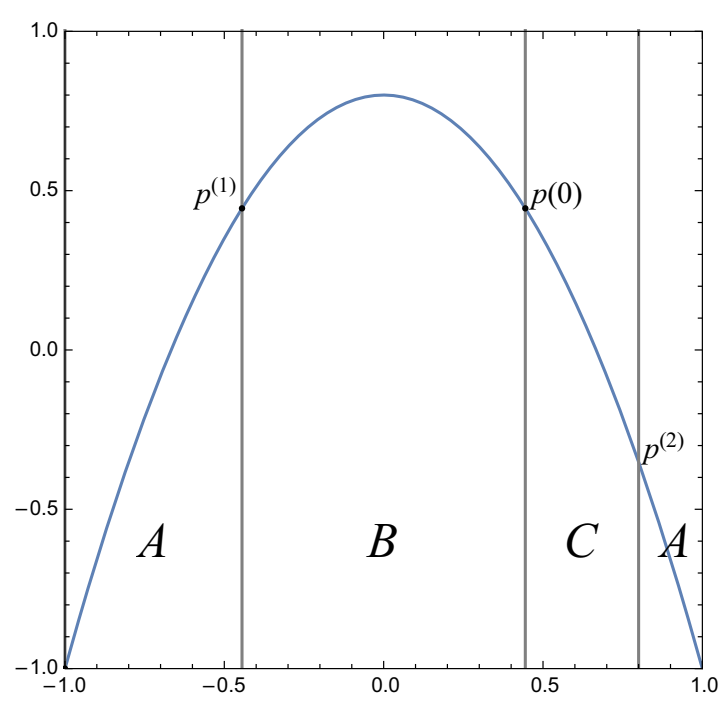

renormalization operator (Proposition 3.5) and establish an estimate for its derivative (Proposition 3.6).

Let $I=[-1,1]$. A unimodal map is a smooth map $f: I \rightarrow I$ such that

1. the point -1 is the unique fixed point with a positive multiplier,

2. $f(1)=-1$, and

3. the map $f$ has a unique maximum at $c \in \operatorname{int}(I)$ and $c$ is a nondegenerate critical point, i.e. $f^{\prime}(c)=0$ and $f^{\prime \prime}(c) \neq 0$.

The class of analytic unimodal maps $f: I \rightarrow I$ is denoted by $\mathcal{U}$.

For a unimodal map $f \in \mathcal{U}$, let $c^{(0)}=c^{(0)}(f) \in I$ be the critical point of $f$. The critical orbit is denoted by $c^{(n)}=f^{n}\left(c^{(0)}\right)$ for all $n>0$. For each $x \in I$, let $x \rightarrow \hat{x}$ be an order reversing involution on $I$ such that the point $\hat{x} \in I$ satisfies $f(\hat{x})=f(x)$ and $\hat{x} \neq x$ if $x \neq c^{(0)}$.

\subsection{The renormalization of a unimodal map}

To define the period-doubling renormalization operator, we introduce a partition of $I$ that allows us to define the first return map for a renormalizable unimodal map.

Definition 3.1 Assume that $f \in \mathcal{U}$ has a unique fixed point $p(0) \in I$ with a negative multiplier. Let $p^{(1)}=\widehat{p(0)}$ and $p^{(2)}$ be the point such that $f\left(p^{(2)}\right)=$ $p^{(1)}$ and $p^{(2)}>c^{(0)}$. Also, let $A=\left(-1, p^{(1)}\right) \cup\left(p^{(2)}, 1\right), B=\left(p^{(1)}, p(0)\right)$, and $C=\left(p(0), p^{(2)}\right)$. The sets $A=A(f), B=B(f)$, and $C=C(f)$ form a partition of the domain $D \equiv I$. See Fig. 1 for an illustration.

The "renormalizable" property is defined by using the partition elements. 
Definition 3.2 Let $B$ and $C$ be the sets defined above. A unimodal map $f \in \mathcal{U}$ is (period-doubling) renormalizable if $f(B) \subset C$. The class of renormalizable unimodal maps is denoted by $\mathcal{U}^{r}$.

Remark 3.3 There are different ways of defining the invariant intervals $B$ and $C$. One common way is to use the critical orbit. We refer to the papers $[14, \mathrm{p}$. 93], [11, p. 616], and [12, p. 737] for examples. However, the definition cannot be applied to a Hénon-like map because the map does not have a critical point. So here we use the preimages of the fixed points instead to define the invariant intervals. This definition will be generalized to Hénon-like maps by replacing the points by the components of a stable manifold (Definition 4.10).

For a renormalizable unimodal map, an orbit that is not eventually periodic runs according to the diagram.

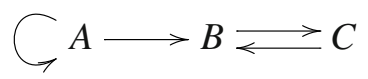

This allows us to define the period-doubling renormalization.

Definition 3.4 Assume that $f \in \mathcal{U}^{r}$. The renormalization of $f$ is the map $R f=s \circ f^{2} \circ s^{-1}$ where $s$ is the orientation-reversing affine rescaling such that $s(p(0))=-1$ and $s\left(p^{(1)}\right)=1$.

The renormalization operator is a map $R: \mathcal{U}^{r} \rightarrow \mathcal{U}$. If the operation of renormalization can be done recurrently infinitely many times, then the map is called infinitely (period-doubling) renormalizable.

\subsection{The fixed point of the renormalization operator}

In this subsection, we study the fixed point $g$ of the renormalization operator. The map $g$ is important in the Hénon case because it also defines the hyperbolic fixed point of the Hénon renormalization operator [11, Theorem 4.1].

The existence and uniqueness of the fixed point were proved in $[8,20]$. Here, the theorem is restated in our coordinate system.

Proposition 3.5 There exist a unique constant $\lambda=2.5029 \ldots$ and a unique solution $g \in \mathcal{I}$ of the Cvitanovic-Feigenbaum-Coullet-Tresser functional equation

$$
g(x)=-\lambda g^{2}\left(-\frac{x}{\lambda}\right)
$$

for $-1 \leq x \leq 1$ with the following properties:

1. $g$ is analytic in a complex neighborhood of $[-1,1]$.

2. $g$ is even.

3. $g$ is concave on $\left[-c^{(1)}, c^{(1)}\right]$. 
4. $g\left(c^{(1)}\right)=-\frac{1}{\lambda} c^{(1)}$ and $g^{\prime}\left(c^{(1)}\right)=-\lambda$.

5. $g$ has negative Schwarzian derivative.

In the remainder of the subsection, the notations for unimodal maps will be applied to the map $g$. For example, $\left\{c^{(j)}=c^{(j)}(g)\right\}_{j \geq 0}$ is the critical orbit and the sets $A=A(g), B=B(g)$, and $C=C(g)$ form a partition of the domain $D=I$.

Next, we show that the map $g$ is expanding on $A$ and $C$.

Proposition 3.6 The inequality

$$
\left|g^{\prime}(x)\right| \geq\left|g^{\prime}(q(0))\right|>1
$$

holds for all $x \in[q(-1), \widehat{q(0)}] \cup[q(0), \widehat{q(-1)}]$.

Proof It is enough to consider $x \in[q(0), \widehat{q(-1)}]$ since $g$ is even.

Let $b^{(1)} \in\left[0, c^{(1)}\right]$ be the point such that $g\left(b^{(1)}\right)=0$. Set $b^{(2)}=\frac{1}{\lambda} b^{(1)}$. Observe that the points form a backward orbit $b^{(2)} \rightarrow b^{(1)} \rightarrow c^{(0)}$ of the critical point. Also, let $q(-1)=-1$ be the fixed point with a positive multiplier and $q(0)$ be the fixed point with a negative multiplier. By (3.1), we have $q(0)=-\frac{1}{\lambda} q(-1)$ and

$$
g^{\prime}(x)=g^{\prime}\left(-\frac{x}{\lambda}\right) g^{\prime} \circ g\left(-\frac{x}{\lambda}\right)
$$

for all $x \in I$. After substituting $x=b^{(1)}$ and $x=q(-1)$ into (3.2), we obtain

$$
g^{\prime}\left(b^{(2)}\right)=-1 \text { and } g^{\prime}(\widehat{q(-1)})=\left[g^{\prime}(q(0))\right]^{2} .
$$

Since $b^{(2)}<q(0)<c^{(1)}, g^{\prime}\left(b^{(2)}\right)=-1$, and $g^{\prime}\left(c^{(1)}\right)=-\lambda$ (Proposition 3.5), we have $\left|g^{\prime}(q(0))\right|>1$ by the minimum principle [17, Lemma 6.1]. We get $\left|g^{\prime}(\widehat{q(-1)})\right|>1$. Therefore, the proposition follows by applying the minimum principle to the interval $[q(0), \widehat{q(-1)}]$.

\section{Hénon-like maps}

In this section, we give an introduction to the framework of Hénon renormalization based on the papers of de Carvalho et al. [11] and Lyubich and Martens [48]. 


\subsection{The class of Hénon-like maps}

Let $I^{v}$ and $I^{h}$ be closed intervals such that $I^{v} \supset I^{h} \ni I \equiv[-1,1]$. A Hénonlike map is a smooth map $F: I^{h} \times I^{v} \rightarrow \mathbb{R}^{2}$ of the form

$$
F(x, y)=(f(x)-\epsilon(x, y), x)
$$

where $f$ is a unimodal map and $\epsilon$ is a small perturbation. The map $F$ will be expressed as $F=(f-\epsilon, x)$. Let $h$ be the $x$-component of the expression, i.e. $h_{y}(x)=h(x, y)=\pi_{x} F(x, y)$. In this paper, we focus on the class of Hénon-like maps satisfying some regularity conditions.

Let $\delta>0$ and $\kappa>0$. Denote by $\mathcal{U}_{\delta, \kappa}\left(I^{h}\right)$ the class of analytic unimodal maps $f: I^{h} \rightarrow \mathbb{R}$ satisfying the following properties:

1. $f$ has a unique nondegenerate critical point $c$ such that $f(c) \in[c+\kappa, 1-\kappa]$,

2. $f$ has two fixed points -1 and $p$ such that -1 has an expanding positive multiplier and $p$ has a negative multiplier,

3. $f$ has a holomorphic extension to $I^{h}(\delta)$,

4. $f$ can be factorized as $f=Q \circ \phi$ where $Q(x)=c^{(1)}-\left(c^{(1)}+1\right) x^{2}, c^{(1)}$ is the critical value, and $\phi$ is an $\mathbb{R}$-symmetric univalent map on $I^{h}(\delta)$, and 5. $f$ has negative Schwarzian derivative.

In the remaining part of the article, we let $\kappa>0$ be a small constant that the class contains the renormalization fixed point $g$, and we suppress $\kappa$ from the notation, i.e. $\mathcal{U}_{\delta}\left(I^{h}\right)=\mathcal{U}_{\delta, \kappa}\left(I^{h}\right)$.

Remark 4.1 The conditions $f(-1)=-1$ and $f(1)=-1$ force $\phi(-1)=-1$ and $\phi(1)=1$. Thus, $\mathcal{U}_{\delta}$ is a normal family by [56, Theorem 3.2].

The function spaces of Hénon-like maps that we will use are defined as follows.

1. Denote by $\mathcal{H}_{\delta}\left(I^{h} \times I^{v}\right)$ the class of real analytic Hénon-like maps $F$ : $I^{h} \times I^{v} \rightarrow \mathbb{R}^{2}$ with the following properties:

(a) $F=(f-\epsilon, x)$ with $f \in \mathcal{U}_{\delta}\left(I^{h}\right)$.

(b) It has a saddle fixed point $p(-1)$ near the point $(-1,-1)$. The fixed point has an expanding positive multiplier.

(c) The map $h \equiv \pi_{x} F$ has a holomorphic extension to $I^{h}(\delta) \times I^{v}(\delta) \rightarrow \mathbb{C}$. 2. Let $\bar{\epsilon}>0$. Denote by $\mathcal{H}_{\delta}\left(I^{h} \times I^{v}, \bar{\epsilon}\right)$ the class of Hénon-like maps $F=$ $(f-\epsilon, x) \in \mathcal{H}_{\delta}\left(I^{h} \times I^{v}\right)$ such that $\|\epsilon\|<\bar{\epsilon}$.

We will use the notations $\delta, I^{h}$ (the horizontal domain), and $I^{v}$ (the vertical domain) in the statements without redefining them.

Remark 4.2 The domain $I^{h} \times I^{v}$ is larger than the domain studied in [11, 48]. Their domain is equivalent to the dynamical interval $\left[f^{2}(c), f(c)\right]$ for 
unimodal maps which does not include the fixed point with an expanding positive multiplier. The larger domain is necessary in this article to study the rescaled orbit of a point. See Propositions 4.7, 4.11, and 5.2.

The theorems in $[11,48]$ also hold on the extended domain $I^{h} \times I^{v}$. See for examples [11, Footnote 7, Subsection 3.4] and [48, Lemma 3.3, Proposition 3.5, Theorem 4.1]. However, re-proving their theorem on the larger domain is not the aim here. Here, we assume the results from $[11,48]$ also hold in the extended domain and rephrase them in the notations used in this article without re-proving them. See also Remarks 4.13, 4.19, and 10.15.

Example 4.3 A map $F=(f-\epsilon, x)$ is called a degenerate Hénon-like map if $\frac{\partial \epsilon}{\partial y}=0$, and a nondegenerate Hénon-like map if $\frac{\partial \epsilon}{\partial y} \neq 0$. If $F$ is degenerate, then $\epsilon$ depends only on $x$. Without loss of generality, we assume the map has the representation $F=(f, x)$ where $f=\pi_{x} F$ and $\epsilon=0$. The dynamics of a degenerate Hénon-like map is completely determined by its unimodal component $f$. So it will also be called the unimodal case.

The degenerate case is an important example. In Sect. 7, we give a proof of the nonexistence of wandering intervals for unimodal maps by identifying a unimodal map with a degenerate Hénon-like map. The expansion estimate used in the proof inspires the proof for the nondegenerate case. The difference between the degenerate case and the nondegenerate case shows the main difficulty (explained in Sects. 8 and 10) in extending the proof to the nondegenerate case.

Example 4.4 The classical Hénon family is the two-parameter family $F_{a, b}(x, y)=\left(-1+a\left(1-x^{2}\right)-b y, x\right)$ where $a, b>0$. These are Hénon-like maps.

\subsection{The local stable manifolds and the partition for a Hénon-like map}

To study the dynamics of a Hénon-like map, we need to define a domain $D \subset$ $I^{h} \times I^{v}$ that turns the Hénon-like map into a self-map. Also, to renormalize a Hénon-like map, we need to find a subdomain $C \subset D$ that is invariant under some iterations. Inspired from unimodal maps, we construct a partition of the domain $I^{h} \times I^{v}$. In the unimodal case, an orbit that maps to the fixed point $p(0)$ splits the domain $D$ into a partition $\{A, B, C\}$ (Definition 3.1). For a strongly dissipative Hénon-like map, the definition is generalized by replacing the orbit by the components of the stable manifold of the saddle fixed point $p(0)$. The components are vertical graphs, which divide the domain into multiple vertical strips.

Definition 4.5 A set $\Gamma$ is a vertical graph if there exists a continuous function $\gamma: I^{v} \rightarrow I^{h}$ such that $\Gamma=\left\{(\gamma(t), t) ; t \in I^{v}\right\}$. The vertical graph $\Gamma$ is said to have Lipschitz constant $L$ if the function $\gamma$ is Lipschitz with constant $L$. 


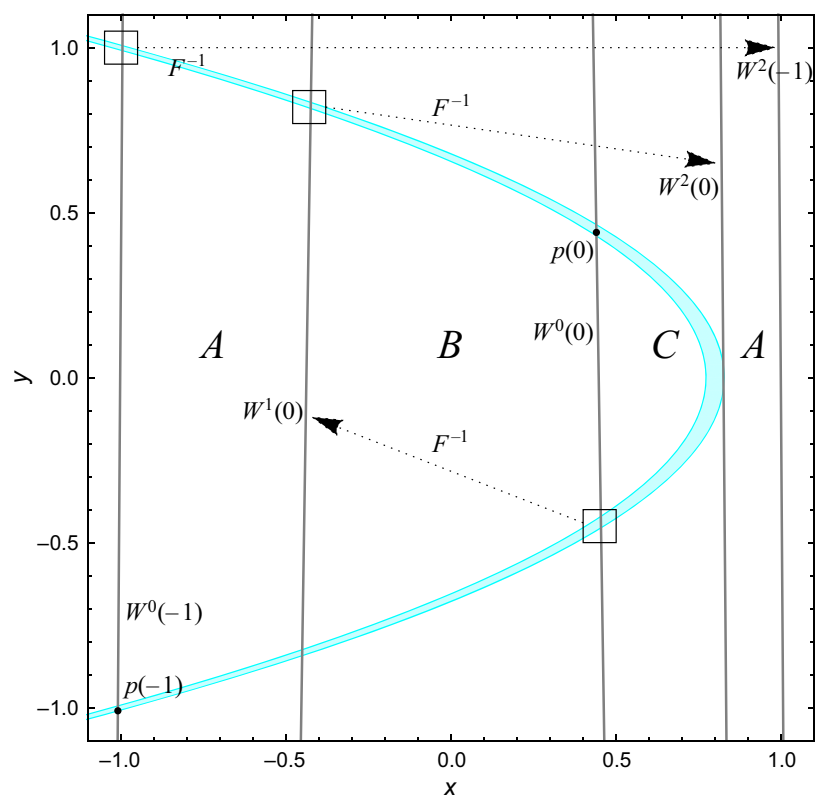

Fig. 2 The local stable manifolds and the partition for a Hénon-like map $F$. The shaded area is the image of the Hénon-like map. The arrows indicate how the local stable manifolds are constructed

In this paper, a local stable manifold is a connected component of a stable manifold. Inspired by [11], the partition elements are defined by the vertical strips separated by the associated local stable manifolds which are vertical graphs.

First, we study the local stable manifolds of the saddle fixed point $p(-1)$ with an expanding positive multiplier.

Definition 4.6 Let $F \in \mathcal{H}_{\delta}\left(I^{h} \times I^{v}\right)$. Consider the stable manifold of the saddle fixed point $p(-1)$.

1. If the connected component that contains the fixed point $p(-1)$ is a vertical graph, let $W^{0}(-1)$ be the component.

2. Suppose that $F^{-1}\left(W^{0}(-1)\right)$ has two components: one is $W^{0}(-1)$ and the other one is a vertical graph. Let $W^{2}(-1)$ be the component that is disjoint from $W^{0}(-1)$.

If the local stable manifolds $W^{0}(-1)$ and $W^{2}(-1)$ exist, let $D=D(F) \subset$ $I^{h} \times I^{v}$ be the open set bounded between the two local stable manifolds. See Fig. 2 for an illustration.

The domain $D$ turns the Hénon-like map into a self-map.

Proposition 4.7 There exist constants $\bar{\epsilon}>0$ and $c>0$ such that the following properties hold for all $F \in \mathcal{H}_{\delta}\left(I^{h} \times I^{v}, \bar{\epsilon}\right)$ : 
1. The sets $W^{0}(-1), W^{2}(-1)$, and $D$ exist. The two local stable manifolds are vertical graphs with Lipschitz constant $c\|\epsilon\|$.

2. $F(D) \subset D$.

Proof first property follows from the graph transformation. The techniques were developed in [48, Section 3]. See [48, Lemma 3.1, 3.2].

The second property follows from the definition of the local stable manifolds by taking $\bar{\epsilon}>0$ to be sufficiently small.

Next, we study the local stable manifolds of the other saddle fixed point $p(0)$ with an expanding negative multiplier to define a partition of $D$.

Definition 4.8 Let $F \in \mathcal{H}_{\delta}\left(I^{h} \times I^{v}\right)$. Assume that $F$ has a saddle fixed point $p(0)$ with an expanding negative multiplier. Consider the stable manifold of $p(0)$.

1. If the connected component that contains $p(0)$ is a vertical graph, let $W^{0}(0)$ be the component.

2. Suppose that $F^{-1}\left(W^{0}(0)\right)$ has two components: one is $W^{0}(0)$ and the other one is a vertical graph. Let $W^{1}(0)$ be the component that is disjoint from $W^{0}(0)$.

3. Suppose that $F^{-1}\left(W^{1}(0)\right)$ has a component that is a vertical graph located between $W^{0}(0)$ and $W^{2}(-1)$. Let $W^{2}(0)$ be the component.

See Fig. 2 for an illustration.

Remark 4.9 At this moment, the numbers 0 and -1 in the notation of the fixed points $p(0)$ and $p(-1)$ (and also the local stable manifolds) do not have a special meaning. After introducing infinitely renormalizable Hénonlike maps, the notation $p(k)$ will be used to define a periodic point with period $2^{k}$. See Definition 5.1. The numbers are introduced here for consistency.

The local stable manifolds split the domain $D$ into vertical strips. These strips define a partition of the domain.

Definition 4.10 Let $F \in \mathcal{H}_{\delta}\left(I^{h} \times I^{v}\right)$. Assume that $F$ has a saddle fixed point $p(0)$ with an expanding negative multiplier, the local stable manifolds in Definition 4.8 exist, and $D$ exists.

1. Let $A=A(F) \subset I^{h} \times I^{v}$ be the union of two sets. One is the open set bounded between $W^{0}(-1)$ and $W^{1}(0)$; the other is the open set bounded between $W^{2}(0)$ and $W^{2}(-1)$.

2. Let $B=B(F) \subset I^{h} \times I^{v}$ be the open set bounded between $W^{0}(0)$ and $W^{1}(0)$.

3. Let $C=C(F) \subset I^{h} \times I^{v}$ be the open set bounded between $W^{0}(0)$ and $W^{2}(0)$. 
For a strongly dissipative Hénon-like map, the local stable manifolds are vertical graphs and the dynamics on the partition is similar to the unimodal case.

Proposition 4.11 There exist constants $\bar{\epsilon}>0$ and $c>0$ such that the following properties hold for all $F \in \mathcal{H}_{\delta}\left(I^{h} \times I^{v}, \bar{\epsilon}\right)$ :

1. The sets $W^{0}(0), W^{1}(0), W^{2}(0), A, B$, and $C$ exist. The local stable manifolds are vertical graphs with Lipschitz constant $c\|\epsilon\|$.

2. $F(A) \subset A \cup W^{1}(0) \cup B$.

3. $F(C) \subset B$.

4. If $z \in A$, then there exists $n>0$ such that $F^{n}(z) \notin A$.

Proof The first property is proved by graph transformation. See [48, Section 3].

The second and third properties follows from the definition of the local stable manifolds. See also [48, Lemma 4.2].

The last property holds because the only fixed points are $p(-1)$ and $p(0)$ so the local unstable manifold of $p(-1)$ must extends across the whole set $A$. See also [48, Lemma 4.2].

By the definition of $B$, its iterate $F(B)$ is contained in the right component of $D \backslash W^{0}(0)$. With the third property of Proposition 4.11, we can define what it means for a Hénon-like map to be renormalizable.

Definition 4.12 Assume that $\bar{\epsilon}>0$ is small. A Hénon-like map $F \in \mathcal{H}_{\delta}\left(I^{h} \times\right.$ $I^{v}, \bar{\epsilon}$ ) is (period-doubling) renormalizable if $F(B) \subset C$. The class of renormalizable Hénon-like maps is denoted by $\mathcal{H}_{\delta}^{r}\left(I^{h} \times I^{v}, \bar{\epsilon}\right) \subset \mathcal{H}_{\delta}\left(I^{h} \times I^{v}, \bar{\epsilon}\right)$.

Remark 4.13 The definition of "renormalizable" in this paper is similar to [11, Subsection 3.4] (called pre-renormalization there) but not exactly the same. The "renormalizable" condition in [11] will be called CLM-renormalizable here. Under the definition of a CLM-renormalizable map, the set " $C$ ", where the points are rescaled, is the region bounded between $W^{0}(0)$ and a segment of the unstable manifold of $p(-1)$. In this article, the set $C$ is defined to be the largest candidate (around the critical value) that is invariant under $F^{2}$ which only uses the local stable manifolds of $p(0)$. Thus, the sets $B$ and $C$ for a renormalizable Hénon-like map are larger than the sets for a CLMrenormalizable map.

As a result, the "renormalizable" property in this article is stronger than in [11]. If a Hénon-like map is renormalizable, then it is also CLMrenormalizable. Although the converse is not true in general, the hyperbolicity of the renormalization operator [11, Theorem 4.1] allows us to apply renormalization to an infinitely CLM-renormalizable map. This makes the final conclusion, Theorem 10.14, also work for CLM-renormalizable maps. See Remarks 4.19 and 10.15 for more details. 
Their definition has some advantages and disadvantages. The definition of CLM-renormalizable is independent of the size of the vertical domain $I^{v}$. However, their sets $B$ and $C$ are too small. It may require more iterations for an orbit to enter their $B$ and $C$. See the proof of [48, Lemma 4.2]. This is the reason for adjusting their definition.

For a renormalizable Hénon-like map, an orbit that is disjoint from the stable manifolds runs according to the diagram.

$$
\text { finite iterations } C A \longrightarrow B \rightleftarrows C
$$

Therefore, a renormalizable map has a first return map to $C$.

\subsection{The renormalization operator}

When a Hénon-like map is renormalizable, the map has a first return map to $C$. However, the first return map is no longer a Hénon-like map by a direct computation

$$
F^{2}(x, y)=\left(h_{x}\left(h_{y}(x)\right), h_{y}(x)\right) .
$$

The paper [11] introduced the nonlinear coordinate change $H(x, y) \equiv$ $\left(h_{y}(x), y\right)$ that turns the first return map into a Hénon-like map. The next proposition defines the renormalization operator.

Proposition 4.14 (The renormalization operator) There exist constants $\bar{\epsilon}>0$ and $c>0$ such that for all $F \in \mathcal{H}_{\delta}^{r}\left(I^{h} \times I^{v}, \bar{\epsilon}\right)$ there exists an $\mathbb{R}$-symmetric orientation reversing affine map $s=s(F)$, which depends continuously on $F$, satisfying the following properties:

$$
\text { Let } \Lambda(x, y)=(s(x), s(y)) \text { and } \phi=\Lambda \circ H \text {. }
$$

1. The map $x \rightarrow h_{y}(x)$ is injective on a neighborhood of $C(F)$ and hence $\phi$ is a diffeomorphism from the neighborhood to its image.

2. The renormalization $R F \equiv \phi \circ F^{2} \circ \phi^{-1}$ is an Hénon-like map defined on $I_{R}^{h}\left(\delta_{R}\right) \times I_{R}^{v}\left(\delta_{R}\right)$ for some $\delta_{R}>0$ and intervals $I_{R}^{h}$ and $I_{R}^{v}$. The intervals satisfy $I_{R}^{h} \ni[-1,1]$ and $I_{R}^{v}=s\left(I^{v}\right)$.

3. The domain $I_{R}^{h} \times I_{R}^{v}$ contains $D(R F)$, and $\phi(C(F))=D(R F)$.

4. The fixed points satisfy the relation $\phi(p(0))=p_{R F}(-1)$ where $p_{R F}(-1)$ is the saddle fixed point of $R F$ with an expanding positive multiplier.

5. The renormalization has a representation $R F=\left(f_{R}-\epsilon_{R}, x\right)$ where $f_{R} \in$ $\mathcal{U}$. The representation satisfies the relations

$$
\left\|f_{R}-R_{c} f\right\|_{I_{R}^{h}\left(\delta_{R}\right)}<c\|\epsilon\|
$$

and 


$$
\left\|\epsilon_{R}\right\|_{I_{R}^{h}\left(\delta_{R}\right) \times I_{R}^{v}\left(\delta_{R}\right)}<c\|\epsilon\|^{2} .
$$

Proof See [11, Subsection 3.5].

Remark 4.15 The rescaling $\phi$ preserves the orientation along the $x$-coordinate and reverses the orientation along the $y$-coordinate.

A map is called infinitely renormalizable if the operation of renormalization can be done infinitely many times. The class of infinitely renormalizable Hénon-like maps is denoted by $\mathcal{I}_{\delta}\left(I^{h} \times I^{v}, \bar{\epsilon}\right) \subset \mathcal{H}_{\delta}\left(I^{h} \times I^{v}, \bar{\epsilon}\right)$.

Assume that $F \in \mathcal{I}_{\delta}\left(I^{h} \times I^{v}, \bar{\epsilon}\right)$, we define $F_{n}=R^{n} F$. The subscript $n$ is called the renormalization scale. The subscript is also used to indicate the associated renormalization scale of an object. For example, $F_{n}$ is defined on the domain $I^{h} \times I_{n}^{v}$. The maps $H_{n}, s_{n}$, and $\Lambda_{n}$ are the rescaling maps for $F_{n}$ defined in Proposition 4.14. The vertical domains $\left\{I_{n}^{v}\right\}_{n}$ satisfy $I_{0}^{v}=I^{v}$ and $I_{n+1}^{v}=s_{n}\left(I_{n}^{v}\right)$ for all $n \geq 0$. Let $\lambda_{n}=\left|s_{n}^{\prime}(x)\right|$ be the rescaling factor of the affine rescaling map $s_{n}$. The points $p_{n}(-1)$ and $p_{n}(0)$ are the two saddle fixed points of $F_{n}$. The vertical graphs $\left\{W_{n}^{t}(j)\right\}_{t}$ are the local stable manifolds of the period point $p_{n}(j)$ for $j=-1,0$. The sets $A_{n}, B_{n}$, and $C_{n}$ form a partition of the dynamical domain $D_{n}$ for $F_{n}$.

The renormalization operator is hyperbolic. The next proposition lists the properties of infinitely renormalizable Hénon-like maps. Recall from Proposition 3.5 that $g \in \mathcal{U}$ is the fixed point of the unimodal renormalization operator, and $\lambda$ is the rescaling constant. Let $G(x, y)=(g(x), x)$ be the induced degenerate Hénon-like map.

Proposition 4.16 (The hyperbolicity of the renormalization operator) There exist constants $\rho<1$ (universal), $\bar{\epsilon}>0$, and $c>0$ such that for all $F \in$ $\mathcal{I}_{\delta}\left(I^{h} \times I^{v}, \bar{\epsilon}\right)$ there exist a constant $\delta_{R}$ with $0<\delta_{R}<\delta$, an interval $I_{R}^{h}$ with $I^{h} \supset I_{R}^{h} \ni I$, and a constant $b=b(F) \in \mathbb{R}$ such that the following properties hold:

Let $F_{n}=R^{n} F$. Then $F_{n} \in \mathcal{H}_{\delta_{R}}\left(I_{R}^{h} \times I_{n}^{v}\right)$ for all $n \geq 0$. Also, the sequence has an expression $F_{n}=\left(f_{n}-\epsilon_{n}, x\right)$ with $f_{n} \in \mathcal{U}_{\delta_{R}}\left(I_{R}^{h}\right)$ that satisfies
1. $\left\|f_{n}-g\right\|_{I_{R}^{h}\left(\delta_{R}\right)}<c \rho^{n}\|F-G\|_{I_{R}^{h}\left(\delta_{R}\right) \times I^{v}\left(\delta_{R}\right)}$
2. $\left\|\epsilon_{n+1}\right\|_{I_{R}^{h}\left(\delta_{R}\right) \times I_{n+1}^{v}\left(\delta_{R}\right)}<c\left\|\epsilon_{n}\right\|_{I_{R}^{h}\left(\delta_{R}\right) \times I_{n}^{v}\left(\delta_{R}\right)}^{2}$,
3. $\left\|f_{n+1}-s_{n} \circ f_{n}^{2} \circ s_{n}^{-1}\right\|_{I_{R}^{h}\left(\delta_{R}\right)}<c\left\|\epsilon_{n}\right\|_{I_{R}^{h}\left(\delta_{R}\right) \times I_{n}^{v}\left(\delta_{R}\right)}$,
4. $\left|\lambda_{n}-\lambda\right|<c \rho^{n}\|F-G\|_{I_{R}^{h}\left(\delta_{R}\right) \times I^{v}\left(\delta_{R}\right)}$, and
5. $\epsilon_{n}(x, y)=b^{2^{n}} a(x) y\left(1+O\left(\rho^{n}\right)\right)$ (universality)

for all $n \geq 0$ where $a(x)$ is a universal analytic positive function. The value $\delta_{R}$ in the estimates can be replaced by any positive number that is smaller than $\delta_{R}$. 
Proof See [11, Theorems 3.5, 4.1, 7.9, and Lemma 7.4].

Remark 4.17 The constant $b$ is called the average Jacobian of $F$. See [11, Section 6].

Remark 4.18 The Hénon renormalization is an operation that rescales the first return map around the critical value. However, the renormalization $F_{n}$ converges to the fixed point $G$ of the unimodal renormalization that rescales the first return map around the critical point. This is because of the nonlinear rescaling $H$ maps the domain from $C$ to $B$ in the degenerate case. See Sect. 7 for a more detail explanation.

Remark 4.19 Although being infinitely CLM-renormalizable in general does not imply being infinitely renormalizable, the hyperbolicity provides a connection between the two notions of infinitely renormalizable. Assume that $F$ is infinitely CLM-renormalizable. The hyperbolicity of the renormalizable operator [11, Theorem 4.1] says that $R^{n} F$ converges to the fixed point $G$. This means that $R^{n} F$ is also infinitely renormalizable for all $n$ sufficiently large. This makes Theorem 10.14 also apply to infinitely CLM-renormalizable Hénon-like maps. See Remark 10.15 for more details.

From now on, for any infinitely renormalizable map $F$, we fix a representation $F_{n}=\left(f_{n}-\epsilon_{n}, x\right)$ such that the maps $f_{n}$ and $\epsilon_{n}$ satisfy the properties given in Proposition 4.16. Also, we neglect the subscript of the supnorms $\left\|f_{n}-g\right\|=$ $\left\|f_{n}-g\right\|_{I_{R}^{h}\left(\delta_{R}\right)}$ and $\left\|\epsilon_{n}\right\|=\left\|\epsilon_{n}\right\|_{I_{R}^{h}\left(\delta_{R}\right) \times I_{n}^{v}\left(\delta_{R}\right)}$ whenever the context is clear.

Corollary 4.20 There exists a constant $c>1$ such that

$$
\left\|F_{n}-G\right\|<c \rho^{n}\|F-G\| \text { and }\left\|\epsilon_{n+i}\right\|<\left(c\left\|\epsilon_{n}\right\|\right)^{2^{i}}
$$

for all integers $i, n \geq 1$.

Lemma 4.21 There exists constants $\bar{\epsilon}>0$ and $c_{1}>0$ such that

$$
\left|\frac{\partial \epsilon_{n}}{\partial x}(x, y)\right|,\left|\frac{\partial \epsilon_{n}}{\partial y}(x, y)\right| \leq c_{1}\left\|\epsilon_{n}\right\|
$$

for all $F \in \mathcal{I}_{\delta}\left(I^{h} \times I^{v}, \bar{\epsilon}\right)$ and $(x, y) \in I^{h} \times I_{n}^{v}$. In addition, if $F$ is nondegenerate, there exist constants $N=N(F) \geq 0$ and $c_{2}>0$ such that

$$
\left|\frac{\partial \epsilon_{n}}{\partial y}(x, y)\right| \geq \frac{c_{2}}{\left|I_{n}^{v}\right|}\left\|\epsilon_{n}\right\|
$$

for all $(x, y) \in I^{h} \times I_{n}^{v}$ and $n \geq N$. 
Proof The first inequality (4.1) follows from (2.1).

By the universality (Proposition 4.16:5) of infinitely renormalizable Hénonlike maps, the perturbation $\epsilon$ and its derivative have the asymptotic form

$$
\epsilon_{n}(x, y)=b^{2^{n}} a(x) y\left(1+O\left(\rho^{n}\right)\right)
$$

and thus

$$
\frac{\partial \epsilon_{n}}{\partial y}(x, y)=b^{2^{n}} a(x)\left(1+O\left(\rho^{n}\right)\right) .
$$

Since $a$ is a positive universal map on a compact set that covers the whole domain, the second inequality follows.

To study wandering domains, it is enough to consider Hénon-like maps that are close to the hyperbolic fixed point $G$. By Corollary 6.4 below, an infinitely renormalizable Hénon-like map $F$ has a wandering domain in $D(F)$ if and only if $F_{n}$ has a wandering domain in $D\left(F_{n}\right)$ for all $n \geq 0$. Also, $F_{n} \rightarrow G$ as $n \rightarrow \infty$ by Proposition 4.16. Thus, we may focus on a small neighborhood of the fixed point $G$.

Definition 4.22 If $\bar{\epsilon}$ is small enough such that Proposition 4.16 holds, let $\hat{\mathcal{I}}_{\delta}\left(I^{h} \times I^{v}, \bar{\epsilon}\right)$ be the class of nondegenerate Hénon-like maps $F \in \mathcal{I}_{\delta}\left(I^{h} \times\right.$ $\left.I^{v}, \bar{\epsilon}\right)$ such that $F_{n} \in \mathcal{H}_{\delta}\left(I^{h} \times I_{n}^{v}, \bar{\epsilon}\right),\left\|F_{n}-G\right\|<\bar{\epsilon},\left|\lambda_{n}-\lambda\right|<\bar{\epsilon}$, $\left\|s_{n}(x)-(-\lambda) x\right\|_{I^{h}}<\bar{\epsilon}$, and (4.2) holds for all $n \geq 0$.

In the remaining part of the article, we will study the dynamics and the topology of Hénon-like maps in a small neighborhood of the renormalization limiting map given by Definition 4.22.

\section{The structure and dynamics of infinitely renormalizable Hénon-like maps}

In this section, we study the topology of the local stable manifolds and the dynamics on the partition for an infinitely renormalizable Hénon-like map.

\subsection{The rescaling levels}

In this subsection, we introduce a finer partition of $C$, called the rescaling levels, based on the maximum number of possible rescalings of a point in $C$.

Definition 5.1 Let $\Phi_{n}^{j}=\phi_{n+j-1} \circ \cdots \circ \phi_{n}$ and $C_{n}(0)=A_{n} \cup W_{n}^{1}(0) \cup B_{n}$. The $j$-th rescaling level in $C$ is $C_{n}(j)=\left(\Phi_{n}^{j}\right)^{-1}\left(C_{n+j}(0)\right)$ and the $j$-th rescaling 
level in $B$ is $B_{n}(j)=F_{n}^{-1}\left(C_{n}(j)\right)$. Let $p_{n}(j)=\left(\Phi_{n}^{j}\right)^{-1}\left(p_{n+j}(0)\right)$ and $W_{n}^{t}(j)$ $=\left(\Phi_{n}^{j}\right)^{-1}\left(W_{n+j}^{t}(0)\right)$ for $t=0,2$.

According to the definition of renormalization and rescaling levels, the following diagram commutes.

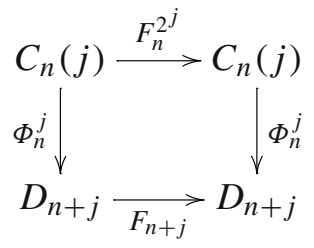

Thus, the dynamical objects in different renormalization scales are related by the following proposition.

Proposition 5.2 There exists a constant $\bar{\epsilon}>0$ such that the following properties hold for all $F \in \hat{\mathcal{I}}_{\delta}\left(I^{h} \times I^{v}, \bar{\epsilon}\right)$ and $n \geq 0$ :

1. $p_{n}(j)$ is a periodic point of $F_{n}$ with period $2^{j}$ for $j \geq 0$.

2. $W_{n}^{t}(j)$ is a local stable manifold of $p_{n}(j)$ for $j \geq 0$ and $t=0,2$.

3. $\Phi_{n}^{k}\left(W_{n}^{t}(j)\right)=W_{n+k}^{t}(j-k)$ and $\Phi_{n}^{k}\left(p_{n}(j)\right)=p_{n+k}(j-k)$ for $j \geq k-1$ and $t=0,2$.

4. The map $\Phi_{n}^{k}: C_{n}(j) \rightarrow C_{n+k}(j-k)$ is a diffeomorphism for $j \geq k$, and

5. For each $j \geq 0$, the set $C_{n}(j)$ contains two components. The left component $C_{n}^{l}(j)$ is the set bounded between $W_{n}^{0}(j-1)$ and $W_{n}^{0}(j)$ and the right component $C_{n}^{r}(j)$ is the set bounded between $W_{n}^{2}(j)$ and $W_{n}^{2}(j-1)$.

The sets $\left\{C_{n}(j)\right\}_{j \geq 1}$ form a partition of $C_{n}$ and the sets $\left\{B_{n}(j)\right\}_{j \geq 1}$ form a partition of $B_{n}$. The partition and the local stable manifolds are illustrated in Fig. 3.

Next, we introduce the tip to study the geometric structure of the rescaling levels in $C$. Recall from [11, Subsection 7.2] the following definition.

Definition 5.3 Assume that $\bar{\epsilon}>0$ is sufficiently small. The tip of an infinitely renormalizable Hénon-like map $F \in \hat{\mathcal{I}}_{\delta}\left(I^{h} \times I^{v}, \bar{\epsilon}\right)$ is the unique point $\tau$ such that

$$
\{\tau\}=\cap_{j=N}^{\infty}\left(\Phi_{0}^{j}\right)^{-1}\left(D_{j} \cap I^{h} \times I^{h}\right)
$$

for all $N \geq 0$.

The tip is an analogue of the critical value in the nondegenerate case. The closure of its orbit is the attracting Cantor set that is conjugate to the dyadic adding machine. See [11, Section 5] for more information. 


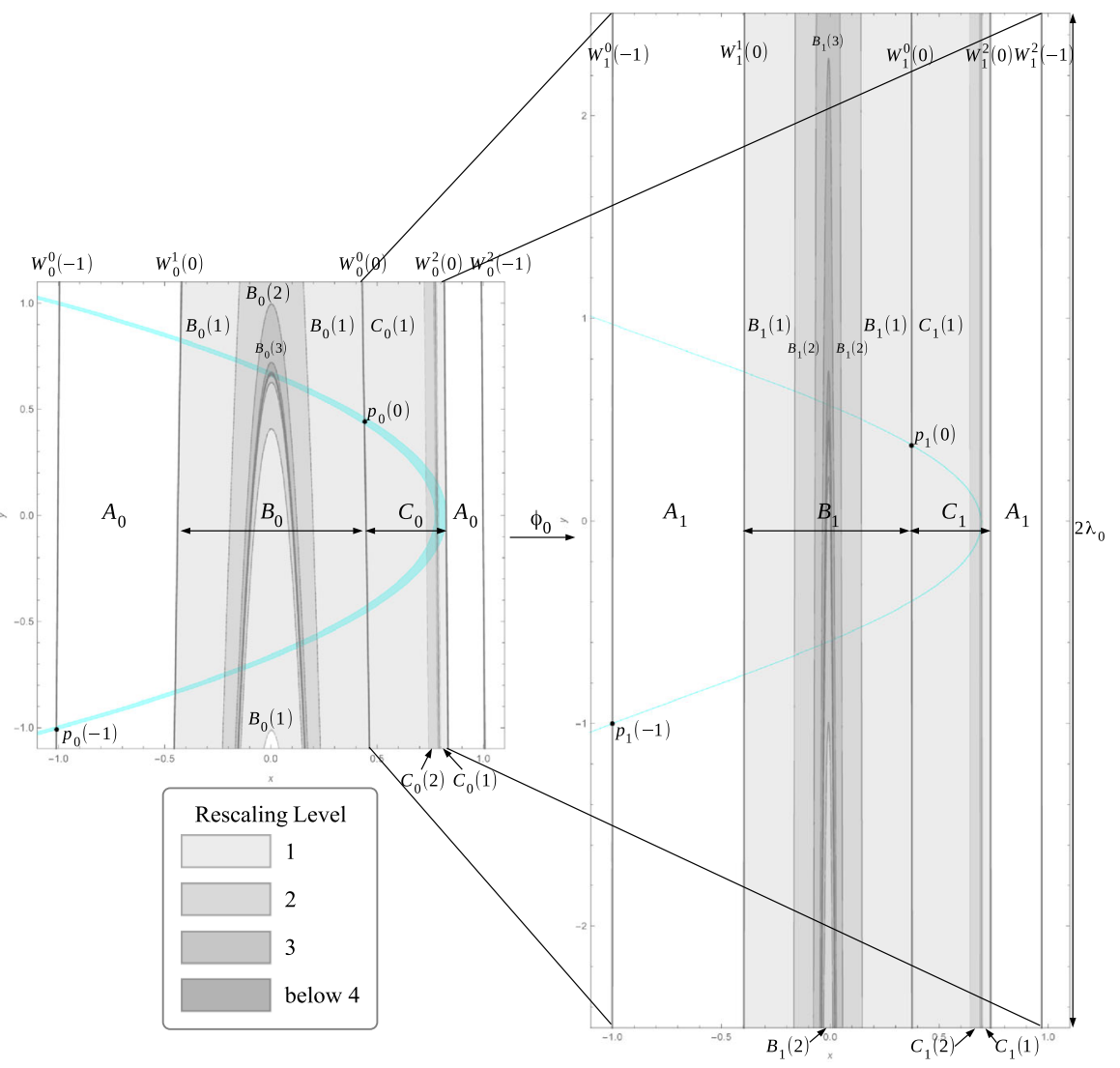

Fig. 3 The partition and the local stable manifolds of two renormalization scales $F_{0}$ and $F_{1}$

A rescaling level $C_{n}(j)$ is the union of two vertical strips. Each of the vertical strips is bounded by two local stable manifolds. Thus, the structure of the rescaling levels is characterized by the structure of the local stable manifolds. Here, we list the geometric properties of the local stable manifolds.

Proposition 5.4 There exist constants $\bar{\epsilon}>0, c>0$, and $c^{\prime}>1$ such that the following properties hold for all $F \in \hat{\mathcal{I}}_{\delta}\left(I^{h} \times I^{v}, \bar{\epsilon}\right), n \geq 0, j \geq-1$, and $t=0,2$ :

1. $W_{n}^{t}(j)$ is a vertical graph with Lipschitz constant $c\left\|\epsilon_{n}\right\|$.

2. $\frac{1}{c^{\prime}}\left(\frac{1}{\lambda}\right)^{2 j}<\left|z_{n}^{(t)}(j)-\tau_{n}\right|<c^{\prime}\left(\frac{1}{\lambda}\right)^{2 j}$ where $z_{n}^{(t)}(j)$ is the intersection point of $W_{n}^{t}(j)$ with the horizontal line through $\tau_{n}$. See Fig. 4.

Proof See [48, Lemma 3.4 and Proposition 3.5]. 


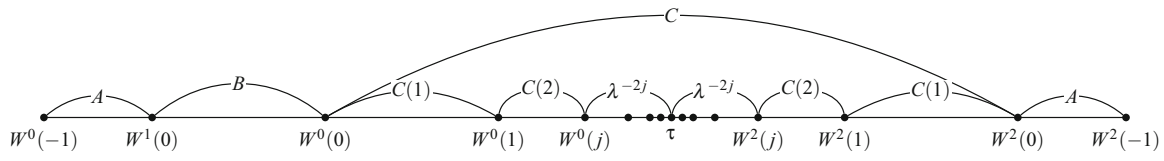

Fig. 4 The structure of the rescaling levels. The figure shows the partition and the local stable manifolds on the horizontal cross section that intersects the tip

\subsection{The behavior of maps near $G$}

In this subsection, we estimate the derivatives of a Hénon-like map which is close to the hyperbolic fixed point $G$. Let $v_{n} \in I^{h}$ be the critical point of $f_{n}$ and $w_{n}=f_{n}\left(v_{n}\right)$ be the critical value.

The first lemma proves that a Hénon-like map acts like a quadratic map on $B$.

Lemma 5.5 There exist $a>0, \bar{\epsilon}>0$, and $I^{B} \subset I^{h}$ such that the following properties hold for all $F \in \hat{\mathcal{I}}_{\delta}\left(I^{h} \times I^{v}, \bar{\epsilon}\right)$ and $n \geq 0$ :

The interior of $I^{B}$ contains $\hat{q}(0)$ and $q(0), I^{B} \times I_{n}^{v} \supset B_{n}$,

$$
\frac{1}{a}\left|x-v_{n}\right| \leq\left|f_{n}^{\prime}(x)\right| \leq a\left|x-v_{n}\right|
$$

and

$$
\frac{1}{2 a}\left(x-v_{n}\right)^{2} \leq\left|f_{n}(x)-f_{n}\left(v_{n}\right)\right| \leq \frac{a}{2}\left(x-v_{n}\right)^{2}
$$

for all $x \in I^{B}$.

Proof The lemma is true because the map $F$ is close to the hyperbolic fixed point $G$ and the map $g$ is concave on the compact set $\left[-c^{(1)}, c^{(1)}\right]$ by Proposition 3.5.

The next lemma shows that a Hénon-like map is expanding on $A$ and $C$ along the $x$-coordinate when the map is close to the fixed point $G$.

Lemma 5.6 There exist $E>1, \bar{\epsilon}>0$, and a union of two intervals $I^{A C} \subset I^{h}$ such that the following properties hold for all $F \in \hat{\mathcal{I}}_{\delta}\left(I^{h} \times I^{v}, \bar{\epsilon}\right)$ and $n \geq 0$ :

The interior of $I^{A C}$ contains $q(-1), \hat{q}(0), q(0)$, and $\hat{q}(-1), I^{A C} \times I_{n}^{v} \supset$ $A_{n} \cup W_{n}^{2}(0) \cup C_{n}$, and

$$
\left|\frac{\partial h_{n}}{\partial x}(x, y)\right| \geq E
$$

for all $(x, y) \in I^{A C} \times I_{n}^{v}$. 
Proof The lemma is true because the map $g$ is expanding on $A$ and $C$ by Proposition 3.6 and the Hénon-like map $F$ is close to the hyperbolic fixed point $G$ of the renormalization operator.

\subsection{A relation between the tip and the critical value}

In Lemma 5.5, we showed that a Hénon-like map behaves like a quadratic map when a point is close to the critical point $v_{n}$ of $f_{n}$ for a particular representation of $F_{n}=\left(f_{n}-\epsilon_{n}, x\right)$. However, the critical point $v_{n}$ and the critical value $w_{n}$ in the estimates depend on the representation. In this subsection, we show that the critical value $w_{n}$ (of any representation) is $\left\|\epsilon_{n}\right\|$-close to the tip $\tau_{n}$ in Proposition 5.10. This allows us to replace $v_{n}$ and $w_{n}$ in the estimates by the representation independent quantity $\tau_{n}$.

To estimate the distance from the tip to the critical value, we write $\tau_{n}=$ $\left(a_{n}, b_{n}\right)$. Since the rescaling $\phi_{n}$ maps a horizontal line to a horizontal line, we focus on the horizontal slice that intersects the tip in each renormalization scale. Define the restriction of the rescaling map $\phi$ to the slice as

$$
\eta_{n}(x)=\pi_{x} \circ \phi_{n}\left(x, b_{n}\right)=s_{n} \circ h_{n}\left(x, b_{n}\right) .
$$

By the definition of the tip, the quantities satisfy the recurrence relations $\phi_{n}\left(\tau_{n}\right)=\tau_{n+1}, \eta_{n}\left(a_{n}\right)=a_{n+1}$, and $s_{n}\left(b_{n}\right)=b_{n+1}$.

First, we prove an estimate that compares the critical values of two neighboring renormalization scales.

Lemma 5.7 There exist constants $\bar{\epsilon}>0$ and $c>0$ such that for all $F \in$ $\hat{\mathcal{I}}_{\delta}\left(I^{h} \times I^{v}, \bar{\epsilon}\right)$, we have

$$
\left|w_{n+1}-\eta_{n}\left(w_{n}\right)\right|<c\left\|\epsilon_{n}\right\|
$$

for all $n \geq 0$.

Proof First, we compare the critical points $v_{n}$ and $v_{n+1}$. By Proposition 4.16, we have

$$
\left\|f_{n+1}^{\prime}-\left(s_{n} \circ f_{n}^{2} \circ s_{n}^{-1}\right)^{\prime}\right\|_{I^{h}}<c\left\|f_{n+1}-s_{n} \circ f_{n}^{2} \circ s_{n}^{-1}\right\|_{I^{h}(\delta)}<c\left\|\epsilon_{n}\right\|
$$

for some constant $c>0$ when $\bar{\epsilon}>0$ is sufficiently small. Since the critical point of the map $f_{n+1}$ is nondegenerate and the map $\left(s_{n} \circ f_{n}^{2} \circ s_{n}^{-1}\right)^{\prime}$ is a small perturbation of $f_{n+1}^{\prime}$, the root $s_{n}\left(v_{n}\right)$ of $\left(s_{n} \circ f_{n}^{2} \circ s_{n}^{-1}\right)^{\prime}$ is also a small perturbation of the root $v_{n+1}$ of $f_{n+1}^{\prime}$. That is, there exists $c^{\prime}>0$ such that

$$
\left|v_{n+1}-s_{n}\left(v_{n}\right)\right| \leq c^{\prime}\left\|\epsilon_{n}\right\| .
$$


The constant $c^{\prime}$ can be chosen to be independent of $F$ because $F$ is close to $G$. The details are left to the reader.

Moreover, by the quadratic estimates in Lemma 5.5, we get

$$
\begin{aligned}
& \left|f_{n+1}\left(v_{n+1}\right)-s_{n} \circ f_{n}^{2}\left(v_{n}\right)\right| \\
& \quad \leq\left|f_{n+1}\left(v_{n+1}\right)-f_{n+1}\left(s_{n}\left(v_{n}\right)\right)\right|+\left|f_{n+1}\left(s_{n}\left(v_{n}\right)\right)-s_{n} \circ f_{n}^{2}\left(v_{n}\right)\right| \\
& \quad \leq \frac{a}{2}\left|v_{n+1}-s_{n}\left(v_{n}\right)\right|^{2}+\left|f_{n+1}\left(s_{n}\left(v_{n}\right)\right)-s_{n} \circ f_{n}^{2} \circ s_{n}^{-1}\left(s_{n}\left(v_{n}\right)\right)\right| \\
& \quad \leq \frac{a c^{\prime 2}}{2}\left\|\epsilon_{n}\right\|^{2}+c\left\|\epsilon_{n}\right\| \\
& \quad \leq c^{\prime \prime}\left\|\epsilon_{n}\right\|
\end{aligned}
$$

for some constant $c^{\prime \prime}>0$.

Finally, we compare the critical values $w_{n}$ and $w_{n+1}$. We have

$$
\begin{aligned}
\left|w_{n+1}-\eta_{n}\left(w_{n}\right)\right| & =\left|f_{n+1}\left(v_{n+1}\right)-s_{n}\left(f_{n}^{2}\left(v_{n}\right)-\epsilon_{n}\left(f_{n}\left(v_{n}\right), b_{n}\right)\right)\right| \\
& \leq\left|f_{n+1}\left(v_{n+1}\right)-s_{n} \circ f_{n}^{2}\left(v_{n}\right)\right|+\lambda_{n}\left|\epsilon_{n}\left(f_{n}\left(v_{n}\right), b_{n}\right)\right| \\
& =\left(c^{\prime \prime}+2 \lambda\right)\left\|\epsilon_{n}\right\|
\end{aligned}
$$

for all $n \geq 0$ whenever $\bar{\epsilon}$ is small enough that $\lambda_{n} \leq 2 \lambda$.

The rescaling maps $\left\{\eta_{n}\right\}_{n \geq 0}$ can be viewed as a nonautonomous dynamical system (a system that depends on time). An orbit is defined as follows.

Definition 5.8 For each $n \geq 1$, let $Y_{n}$ be a complete metric space, $X_{n} \subset Y_{n}$ be a closed subset, and $f_{n}: X_{n} \rightarrow Y_{n+1}$ be a continuous map. A sequence $\left\{x_{n}\right\}_{n=1}^{\infty}$ is an orbit of the nonautonomous system $\left\{f_{n}\right\}_{n=1}^{\infty}$ if $x_{n} \in X_{n}$ and $x_{n+1}=$ $f_{n}\left(x_{n}\right)$ for all $n \geq 1$. A sequence $\left\{x_{n}\right\}_{n=1}^{\infty}$ is an $\epsilon$-orbit of the nonautonomous system $\left\{f_{n}\right\}_{n=1}^{\infty}$ if $x_{n} \in X_{n}$ and $d\left(x_{n+1}, f_{n}\left(x_{n}\right)\right)<\epsilon$ for all $n \geq 1$.

Next, we state the shadowing theorem for nonautonomous systems.

Lemma 5.9 (The shadowing theorem) Let $\left\{f_{n}: X_{n} \rightarrow Y_{n}\right\}_{n=1}^{\infty}$ be the nonautonomous system defined as in Definition 5.8. Suppose that the system has uniform expansion, i.e. there exists a constant $L>1$ such that $d\left(f_{n}(a), f_{n}(b)\right) \geq L \cdot d(a, b)$ for all $a, b \in X_{n}$ and $n \geq 1$. Let $\epsilon>0$. If $\left\{x_{n}\right\}_{n=1}^{\infty}$ is an $\epsilon$-orbit, there exists a unique orbit $\left\{u_{n}\right\}_{n=1}^{\infty}$ such that

$$
d\left(x_{n}, u_{n}\right) \leq \frac{\epsilon}{L-1}
$$

for all $n \geq 1$. In addition, if the domains $\left\{X_{n}\right\}_{n=1}^{\infty}$ are uniformly bounded, then all $\epsilon$-orbits are shadowed to the same orbit. 
This lemma is an analogue of the Anosov's Shadowing Theorem. See [7, Exercise 5.1.3, Corollary 5.3.2] for the version of autonomous systems. The proof is left to the reader.

Lemma 5.7 shows that the sequence of critical values $\left\{w_{n}\right\}_{n=0}^{\infty}$ is an $\epsilon$ orbit of the expanding nonautonomous system $\left\{\eta_{n}\right\}_{n=0}^{\infty}$. Since the domains are uniformly bounded, the $\epsilon$-orbit is shadowed to the unique orbit $\left\{\pi_{x}\left(\tau_{n}\right)\right\}_{n=0}^{\infty}$ by the Shadowing Theorem. This proves the following.

Proposition 5.10 There exist constants $\bar{\epsilon}>0$ and $c>0$ such that for all $F \in \hat{\mathcal{I}}_{\delta}\left(I^{h} \times I^{v}, \bar{\epsilon}\right)$, we have

$$
\left|f_{n}\left(v_{n}\right)-\pi_{x}\left(\tau_{n}\right)\right|<c\left\|\epsilon_{n}\right\|
$$

for all $n \geq 0$.

\section{The closest approach}

The proof of the main theorem begins from this section. For the sake of contradiction, suppose that a Hénon-like map has a wandering domain $J$.

In this section, we introduce a rescaled orbit $\left\{J_{n}\right\}_{n=0}^{\infty}$ of a wandering domain $J$ which is called the $J$-closest approach. Then we define the horizontal size $l_{n}$, the vertical size $h_{n}$, and the rescaling level $k_{n}$ of an element $J_{n}$. In the remaining part of this paper, we will study the expansion and the contraction of the horizontal sizes of the orbit elements.

Recall the definition of wandering domains.

Definition 6.1 Assume that $F \in \mathcal{H}_{\delta}\left(I^{h} \times I^{v}\right), D(F)$ exists, and $F$ is an open map (diffeomorphism from $D(F)$ to the image). A nonempty connected open set $J \subset D(F)$ is a wandering domain of $F$ if the orbit $\left\{F^{n}(J)\right\}_{n \geq 0}$ is disjoint from the stable manifolds of the periodic points.

Remark 6.2 The definition is consistent with the definition of wandering intervals for unimodal maps. In one real dimension (see for instance [17]), a wandering interval is an interval $J$ that satisfies the conditions:

1. The forward iterates $J, f(J), f^{2}(J), \ldots$ are pairwise disjoint.

2. The interval $J$ is disjoint from the basins of periodic orbits.

For unimodal maps, the second condition implies the first one. Thus, we generalize the definition to Hénon-like maps by imposing only the second condition.

We can construct wandering domains by iterating and rescaling an existing one.

Proposition 6.3 There exists a constant $\bar{\epsilon}>0$ such that the following properties hold for all open maps $F \in \mathcal{H}_{\delta}^{r}\left(I^{h} \times I^{v}\right)$ : 
1. A set $J \subset D(F)$ is a wandering domain of $F$ if and only if $F(J)$ is a wandering domain of $F$.

2. A set $J \subset C(F)$ is a wandering domain of $F$ if and only if $\phi(J) \subset D(R F)$ is a wandering domain of $R F$.

Proof The proposition is true because the stable manifold of a periodic orbit is invariant under iteration and the rescaling of a stable manifold is also a stable manifold.

Corollary 6.4 There exists a constant $\bar{\epsilon}>0$ such that for all open maps $F \in \mathcal{H}_{\delta}^{r}\left(I^{h} \times I^{v}, \bar{\epsilon}\right), F$ has a wandering domain in $D(F)$ if and only if $R F$ has a wandering domain in $D(R F)$.

To study the dynamics of a wandering domain, we apply the procedure of renormalization to the orbit. If a wandering domain is contained in $A_{0}$ or $B_{0}$, then its orbit will eventually leave $A_{0}$ and $B_{0}$ and enter $C_{0}$. When the orbit enters $C_{0}$, we rescale the orbit element by the number of its rescaling level $k$. The rescaled set lands on $A_{k}$ or $B_{k}$ of the renormalization scale $k$, and we repeat the process on the scale. This defines a rescaled orbit.

Definition 6.5 Assume that $\bar{\epsilon}>0$ is sufficiently small and $F \in \mathcal{I}_{\delta}\left(I^{h} \times I^{v}, \bar{\epsilon}\right)$. Let $J \subset A \cup B$ be a connected set that is disjoint from the stable manifolds. We define a rescaled orbit of sets $\left\{J_{n}\right\}_{n=0}^{\infty}$ and the associate renormalization scales $\{r(n)\}_{n=0}^{\infty}$ recursively such that $J_{n} \subset A_{r(n)} \cup B_{r(n)}$ for all $n \geq 0$ :

1. Let $J_{0}=J$ and $r(0)=0$.

2. Suppose that $J_{n} \subset A_{r(n)} \cup B_{r(n)}$ is defined. Let $k_{n}$ be the rescaling level of $J_{n}$, i.e. $J_{n} \in B_{r(n)}\left(k_{n}\right)$.

(a) If $J_{n} \subset A_{r(n)}$, let $J_{n+1}=F_{r(n)}\left(J_{n}\right)$ and $r(n+1)=r(n)$.

(b) If $J_{n} \subset B_{r(n)}$, let $J_{n+1}=\Phi_{r(n)}^{k_{n}} \circ F_{r(n)}\left(J_{n}\right)$ and $r(n+1)=r(n)+k_{n}$.

The map from one orbit element to the next one is called one step. It is a composition of one iteration with possibly some rescalings. The sequence $\left\{J_{n}\right\}_{n=0}^{\infty}$ is called the rescaled orbit of $J$ that approaches the tip in the closest possible way, or $J$-closest approach for short.

The itinerary of a closest approach runs according to the diagram.

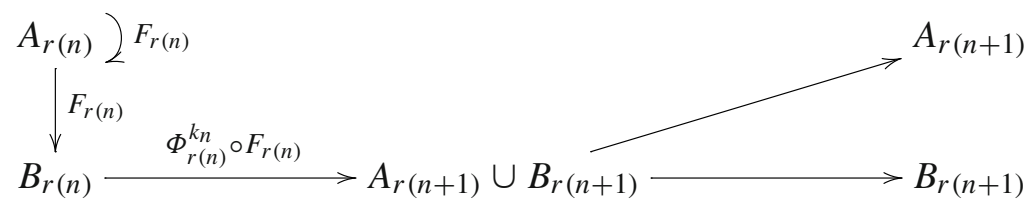

Example 6.6 In this example, we explain the construction of a closest approach and demonstrate the idea used to prove the nonexistence of wandering domains. 


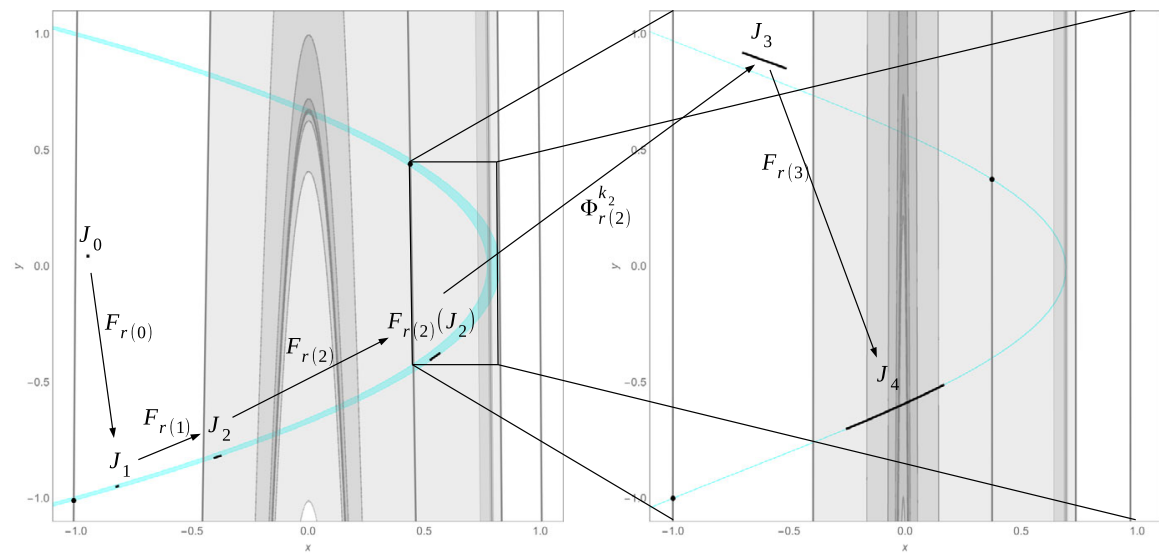

Fig. 5 The construction of a closest approach $J_{n}$. The graphs are the domains and the partitions for $F_{0}$ and $F_{1}$

Assume that $F=(f-\epsilon, x)$ is a Hénon-like map such that $f(x)=$ $1.7996565(1+x)(1-x)-1$ and $\epsilon(x, y)=0.025 y$. The map $F$ is numerically checked to be seven times renormalizable. Let $J=(-0.950,-0.947) \times$ $(0.042,0.045) \subset A$. We show that the set is not a wandering domain by contradiction.

If $J$ is a wandering domain, then we construct a $J$-closest approach as shown in Fig. 5. Let $J_{0}=J$ and $r(0)=0$. The set $J_{0}$ is contained in $A_{r(0)}$. Let $J_{1}=F_{r(0)}\left(J_{0}\right)$ and $r(1)=r(0)=0$. The set $J_{1}$ is also contained in $A_{r(1)}$. Let $J_{2}=F_{r(1)}\left(J_{1}\right)$ and $r(2)=r(1)=0$. The set $J_{2}$ is contained in $B_{r(2)}(1)$. Let $k_{2}=1, r(3)=r(2)+k_{2}=1$, and $J_{3}=\Phi_{r(2)}^{k_{2}} \circ F_{r(2)}\left(J_{2}\right)=\phi_{0} \circ F_{0}\left(J_{2}\right)$. The set $J_{3}$ is contained in $A_{r(3)}$. Let $J_{4}=F_{r(3)}\left(J_{3}\right)$ and $r(4)=r(3)=1$.

From the figure, the sizes of the elements $\left\{J_{n}\right\}$ grow as the construction continues and the size of $J_{4} \subset B_{1}$ becomes so large that the set intersects some stable manifolds. This yields a contradiction. Therefore, $J$ cannot be a wandering domain.

Inspired from the example, we study the growth of horizontal sizes.

Definition 6.7 Assume that $J \subset \mathbb{R}^{2}$. The horizontal size of $J$ is

$$
l(J)=\sup \left\{\left|x_{1}-x_{2}\right| ;\left(x_{1}, y_{1}\right),\left(x_{2}, y_{2}\right) \in J\right\}=\left|\pi_{x} J\right| .
$$

The vertical size of $J$ is

$$
v(J)=\sup \left\{\left|y_{1}-y_{2}\right| ;\left(x_{1}, y_{1}\right),\left(x_{2}, y_{2}\right) \in U\right\}=\left|\pi_{y} J\right| .
$$

If $J$ is compact, a pair of horizontal endpoints are two elements in the set that determines $l(J)$. 
Figure 8 shows a comparison of the sizes of a set $J$. For a Hénon-like map $F \in \mathcal{H}_{\delta}\left(I^{h} \times I^{v}\right)$, it follows from the definition that

$$
v(F(J))=l(J)
$$

for all $J \subset I^{h} \times I^{v}$.

For simplicity, we start from a closed subset $J$ of a wandering domain such that $\overline{\operatorname{int}(J)}=J$ instead of the wandering domain itself. This ensures the horizontal endpoints of an element in the orbit exist. Note that a sequence element $J_{n}$ is also a subset of a wandering domain of $F_{r(n)}$. For elements in a closest approach, let $l_{n}=l\left(J_{n}\right)$ and $v_{n}=v\left(J_{n}\right)$. The reader should not be confused with the subscript of the map $F_{n}$ as the two types of subscript are related by $r(n)$, i.e. $J_{n}$ is in the domain of $F_{r(n)}$. Our aim is to show that $l_{n} \rightarrow \infty$ as $n \rightarrow \infty$ and hence wandering domains cannot exist.

\section{The degenerate case}

In this section, we present a proof of the nonexistence of wandering intervals for the degenerate case (Theorem 7.5). It is known that a unimodal map (under some regularity conditions) does not have wandering intervals $[5,15,16,30,47,52]$. The reader can refer to the article by Guckenheimer [30] for a classical proof. The proof presented here is different from those. We identify unimodal maps with degenerate Hénon-like maps and then use the Hénon renormalization to prove the theorem. This motivates the proof for the nondegenerate case.

\subsection{Local stable manifolds and the partition}

In this subsection, we first identify unimodal maps with degenerate Hénon-like maps and then study the relationships between the two maps.

Let $F$ be a degenerate Hénon-like map

$$
F(x, y)=(f(x), x)
$$

The super-scripts " $u$ " and " $h$ " are used to distinguish the notations between unimodal maps and Hénon-like maps to avoid confusion. For example, $p^{u}(-1)=-1$ and $p^{u}(0)$ are the fixed points of $f ; p^{h}(-1)=(-1,-1)$ and $p^{h}(0)$ are the saddle fixed points of $F ; A^{u}, B^{u}, C^{u} \subset I$ is the partition defined for $f ; A^{h}, B^{h}, C^{h} \subset I^{h} \times I^{v}$ is the partition defined for $F$.

The next lemma relates the local stable manifolds of a degenerate Hénon-like map to the fixed points and their preimages of its unimodal component. Recall that $p^{(1)}$ and $p^{(2)}$ are the points such that $f\left(p^{(2)}\right)=p^{(1)}, f\left(p^{(1)}\right)=p^{u}(0)$, 
and $p^{(1)}<p^{u}(0)<p^{(2)}$ (Definition 3.1); $W^{0}(-1)$ and $W^{2}(-1)$ are the local stable manifolds of $p^{h}(-1)$ (Definition 4.6); $W^{0}(0), W^{1}(0), W^{2}(0)$ are the local stable manifolds of $p^{h}(0)$ (Definition 4.8).

Lemma 7.1 Let $F \in \mathcal{H}_{\delta}\left(I^{h} \times I^{v}\right)$ be a degenerate Hénon-like map. Then

1. $p^{h}(j)=\left(p^{u}(j), p^{u}(j)\right)$ for $j=-1,0$,

2. the local stable manifold $W^{0}(j)$ is the vertical line $x=p^{u}(j)$ for $j=$ $-1,0$,

3. the local stable manifold $W^{2}(-1)$ is the vertical line $x=\widehat{p^{u}(-1)}$,

4. the local stable manifold $W^{1}(0)$ is the vertical line $x=p^{(1)}$,

5. the local stable manifold $W^{2}(0)$ is the vertical line $x=p^{(2)}$,

6. $A^{h}=A^{u} \times I^{v}, B^{h}=B^{u} \times I^{v}, C^{h}=C^{u} \times I^{v}$, and $D^{h}=I \times I^{v}$.

\subsection{The renormalization operator}

Next we relate the Hénon renormalization operator to the unimodal renormalization operator. Recall the definitions of rescaling maps. For a degenerate renormalizable Hénon-like map $F$, the rescaling map is the composition $\phi=\Lambda \circ H$ where $\Lambda(x, y)=\left(s^{h}(x), s^{h}(y)\right), s^{h}$ is the affine rescaling map, and $H(x, y)=(f(x), y)$ is the nonlinear rescaling. The renormalization is $R F=\phi \circ F^{2} \circ \phi^{-1}$. For a renormalizable unimodal map $f, s^{u}$ is the affine rescaling and $R f=s^{u} \circ f^{2} \circ\left(s^{u}\right)^{-1}$ is the renormalization.

Although the Hénon renormalization rescales the first return map around the "critical value", the operation acts like the unimodal renormalization which rescales the first return map around the "critical point". This is because of the nonlinear rescaling $H$ maps the domain around the "critical value" homeomorphically to the domain around the "critical point". Let $\left\{A_{0}^{h}, B_{0}^{h}, C_{0}^{h}\right\}$ be the partition for $F$ and $D_{1}^{h}$ be the domain of $R F$. The rescaling map $\phi(x, y)=\left(s^{h} \circ f(x), s^{h}(y)\right)$ maps $C_{0}^{h}$ homeomorphically to $D_{1}^{h}$. In the $x$ component, the operation $f$ is a bijection from $C_{0}^{u}$ to $B_{0}^{u}$ and the affine map $s^{h}$ rescales $B_{0}^{u}$ back to the unit size $I$. Thus, the two affine maps $s^{u}$ and $s^{h}$ are the same and

$$
H \circ F^{2} \circ H^{-1}(x, y)=\left(\left.f^{2}\right|_{B_{0}^{u}}(x), x\right)
$$

is the first return map to $B_{0}^{h}$. Therefore, the two notions of renormalization coincide

$$
R F(x, y)=\left(s^{u} \circ f^{2} \circ\left(s^{u}\right)^{-1}(x), x\right)=(R f(x), x) .
$$

This also explains why $R^{n} F$ converges to the fixed point $g$ of the unimodal renormalization operator as $n \rightarrow \infty$. 
The observation is summarized in the following proposition.

Proposition 7.2 Let $F \in \mathcal{H}_{\delta}\left(I^{h} \times I^{v}\right)$ be a degenerate Hénon-like map. Then $F$ is Hénon renormalizable if and only if $f$ is unimodal renormalizable. If the maps are renormalizable, then

1. $s^{h}=s^{u}$ and

2. $R F(x, y)=(R f(x), x)$.

In addition, if $F$ is infinitely renormalizable, then the affine rescaling $\Lambda_{n}$ : $B_{n}(j) \rightarrow B_{n+1}(j-1)$ is a bijection for all $n \geq 0$ and $j \geq 1$.

From now on, we remove the super-script from $s$ because the maps are the same.

For an infinitely renormalizable Hénon-like map, we also adapt the subscript used for the renormalization scales to the degenerate case. Assume that a degenerate Hénon-like map $F(x, y)=(f(x), x)$ is infinitely renormalizable. Let $F_{n}=R^{n} F$ and $f_{n}=R^{n} f$. Then $F_{n}(x, y)=\left(f_{n}(x), x\right)$ by the second property of Proposition 7.2.

The expansion estimate in the theorem will be based on the following relation.

Proposition 7.3 (The rescaling trick) Assume that $f$ is infinitely renormalizable. Then

$$
\left(s_{n+j-1} \circ f_{n+j-1}\right) \circ \cdots \circ\left(s_{n} \circ f_{n}\right) \circ f_{n}=f_{n+j} \circ s_{n+j-1} \circ \cdots \circ s_{n}
$$

for all integers $n \geq 0$ and $j \geq 0$.

Proof The proof is by induction on $j$. It is clear that the equality holds when $j=0$.

Assume that the equality holds for some $j$. Then

$$
\begin{aligned}
& \left(s_{n+j} \circ f_{n+j}\right) \circ\left(s_{n+j-1} \circ f_{n+j-1}\right) \circ \cdots \circ\left(s_{n} \circ f_{n}\right) \circ f_{n} \\
& \quad=\left(s_{n+j} \circ f_{n+j}\right) \circ f_{n+j} \circ s_{n+j-1} \circ \cdots \circ s_{n} \\
& \quad=\left(s_{n+j} \circ f_{n+j} \circ f_{n+j} \circ s_{n+j}^{-1}\right) \circ s_{n+j} \circ s_{n+j-1} \circ \cdots \circ s_{n} \\
& \quad=f_{n+j+1} \circ s_{n+j} \circ s_{n+j-1} \circ \cdots \circ s_{n} .
\end{aligned}
$$

Therefore, the lemma is proved by induction.

A similar equality thus holds for degenerate Hénon-like maps.

Corollary 7.4 Let $F \in \mathcal{I}_{\delta}\left(I^{h} \times I^{v}\right)$ be a degenerate Hénon-like map. Then

$$
\Phi_{n}^{j} \circ F_{n}=F_{n+j} \circ \Lambda_{n+j-1} \circ \cdots \circ \Lambda_{n}
$$

for all integers $n \geq 0$ and $j \geq 0$. 


\subsection{Nonexistence of wandering intervals}

In this subsection, we present a proof of the nonexistence of wandering intervals for infinitely renormalizable unimodal maps. Recall that a wandering interval is a nonempty interval such that its orbit elements are disjoint and the omega limit set does not contain a periodic point.

Theorem 7.5 An infinitely renormalizable unimodal map does not have a wandering interval.

Proof Suppose that $f$ is an infinitely renormalizable unimodal map that has a wandering interval $J^{u}$. Without loss of generality, we may assume that the map is close to the fixed point $g$ of the renormalization operator because the sequence of renormalizations $R^{n} f$ converges to $g$ as $n \rightarrow \infty$. Let $F=(f, x)$. Then $F$ is a degenerate infinitely renormalizable Hénon-like map. Assume that $J^{u} \subset I$ is a wandering interval of $f_{0}$. Let $J^{h}=J^{u} \times\{0\}$ and $J_{n} \subset A_{r(n)} \cup B_{r(n)}$ be the $J^{h}$-closest approach. The projection $\pi_{x} J_{n}$ is a wandering interval of $f_{r(n)}$ and the horizontal size $l_{n}$ is the length of the projection. We claim that the horizontal size expands at a definite rate, i.e.

$$
l_{n+1}>E l_{n}
$$

for some constant $E>1$.

If $J_{n} \subset A_{r(n)}$, the inequality (7.1) holds because $g$ is expanding on $A(g)$ by Proposition 3.6 and the map $f_{r(n)}$ is close to $g$.

If $J_{n} \subset B_{r(n)}\left(k_{n}\right)$, then $J_{n+1}=F_{r(n+1)} \circ \Lambda_{r(n)+k_{n}-1} \circ \cdots \circ \Lambda_{r(n)}\left(J_{n}\right)$ by Corollary 7.4. The horizontal size expands when the set $J_{n}$ is mapped by the

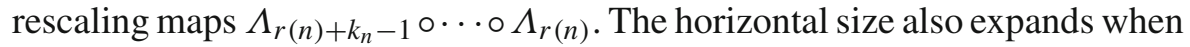
the rescaled set is mapped under $F_{r(n+1)}$. This is because the map $f_{r(n+1)}$ is close to $g, g$ is expanding on $A(g) \cup C(g)$ by Proposition 3.6, and the rescaled set is in $A_{r(n+1)} \cup C_{r(n+1)}$. Thus, the inequality (7.1) also holds.

The expansion estimate (7.1) shows that $l_{n} \rightarrow \infty$ as $n \rightarrow \infty$, which yields a contradiction. Therefore, wandering intervals cannot exist.

In the proof, we showed that the horizontal sizes expand at a definite rate. This inspires the proof for the nondegenerate case. In the remaining part of the article, we will study the growth and contraction rates of the horizontal sizes. We show in Sect. 9 that the expansion estimate can be promoted to nondegenerate maps under some conditions introduced in Sect. 8 . 


\section{The good region and the bad region}

In this section, we group the sub-partitions $\left\{B_{n}(j)\right\}_{j=1}^{\infty}$ and $\left\{C_{n}(j)\right\}_{j=1}^{\infty}$ into two regions by how large $j$ is. The two regions are called the good region and the bad region. Then we study the geometric properties of the regions.

When $j$ is small, the topological structure of the rescaling level $B_{n}(j)$ and the dynamical properties on the level are similar in the degenerate and nondegenerate cases. First, the set $B_{n}(j)$ is the union of two vertical strips. Second, we will show in Sect. 9 that the expansion estimate (7.1) can be generalized to the nondegenerate case on $B_{n}(j)$. The union of the rescaling levels with small values of $j$ is called "the good region".

When $j$ is large, the topological structure of the rescaling level $B_{n}(j)$ and the dynamical properties on the level are different in the two cases. In the degenerate case, the properties are the same as the case when $j$ is small. However, in the nondegenerate case, the set $B_{n}(j)$ becomes an arch-looking connected set and the expansion estimate breaks down on the level. The union of the rescaling levels with large values of $j$ is called "the bad region".

A vertical line argument explains why the expansion estimate breaks down in the bad region. See Fig. 6 for an illustration. First, draw a vertical line (the dashed vertical line in the figure) close to the tip such that the intersection with the image of $F_{n}$ has only one component. We apply the inverse $F_{n}^{-1}$ to the intersection. Unlike the case when the vertical line is far from the tip, the preimage is a concave curve around the center of the domain. Assume that there is a wandering domain $J$ close to the preimage. If the line $\overleftrightarrow{U V}$ connecting the horizontal endpoints $U$ and $V$ of $J$ is parallel to the preimage (Fig. 6a), then the line connecting $F_{n}(U)$ and $F_{n}(V)$ is also parallel to the vertical line (Fig. 6b). Thus, the horizontal size of $F_{n}(J)$ is small and does not depend on the size of $J$.

Inspired by the vertical line argument, we group the rescaling levels in $C$ by how close to the tip a rescaling level is. The size of the image of $F_{n}$ is of order $\left\|\epsilon_{n}\right\|$. Thus, we can expect that the expansion estimate holds when the rescaling level is $>\left\|\epsilon_{n}\right\|$ apart from the tip, and breaks down when the rescaling level is $<\left\|\epsilon_{n}\right\|$ close to the tip. This suggests the definition of the good region and the bad region.

Definition 8.1 Assume that $\bar{\epsilon}>0$ is so small that Proposition 5.4 holds. Let $b>0$ be a constant and $F \in \hat{\mathcal{I}}_{\delta}\left(I^{h} \times I^{v}, \bar{\epsilon}\right)$. For each $n \geq 0$, let $K_{n}=K_{n}(b)$ be the largest positive integer such that

$$
\left|\pi_{x} z_{n}^{(0)}\left(K_{n}\right)-\pi_{x} \tau_{n}\right|>b\left\|\epsilon_{n}\right\|
$$

where $z_{n}^{(0)}(j)$ is the intersection point of $W_{n}^{0}(j)$ with the horizontal line through $\tau_{n}$. 


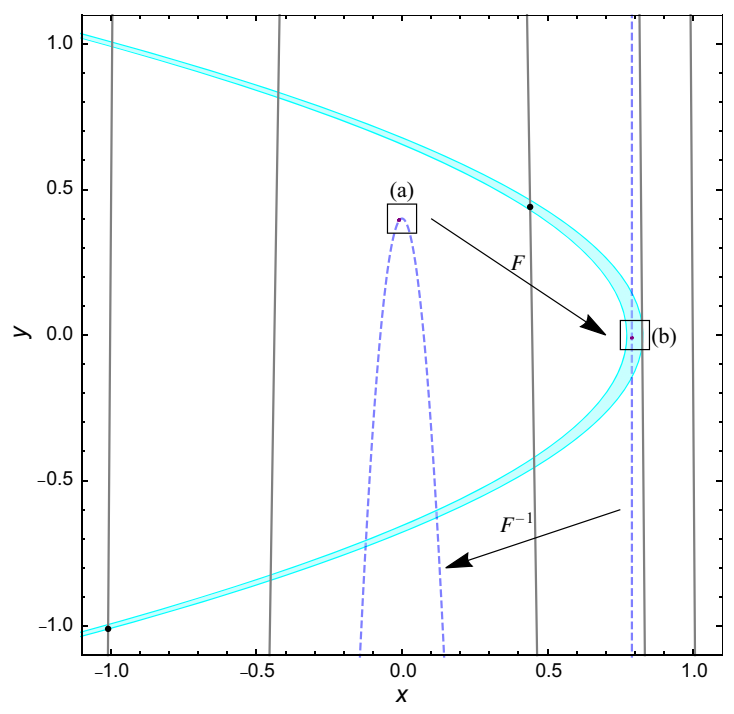

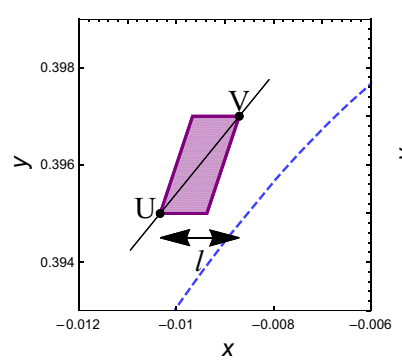

(a) $J$

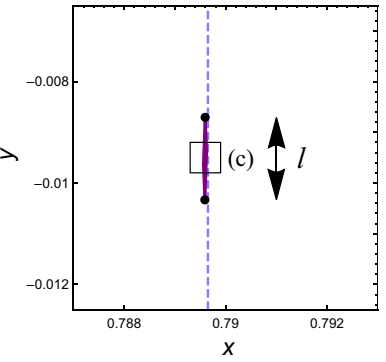

(b) $F_{n}(J)$

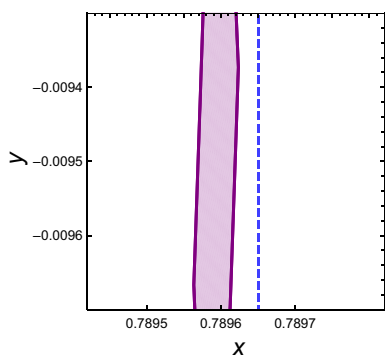

(c) Zoom in (b).

Fig. 6 The vertical line argument

The rescaling level $C_{n}(j)$ (resp. $\left.B_{n}(j)\right)$ is in the good region if $j \leq K_{n}$, and in the bad region if $j>K_{n}$. The integer $K_{n}$ is called the boundary between the regions. See Fig. 7 for an illustration.

In the remaining part of this section, we will study the geometric properties of the regions.

Proposition 8.2 There exist constants $\bar{\epsilon}>0, \bar{b}>0$, and $c>1$ such that for all $F \in \hat{\mathcal{I}}_{\delta}\left(I^{h} \times I^{v}, \bar{\epsilon}\right), b>\bar{b}$, and $n \geq 0$, the boundary $K_{n}$ satisfies

$$
\frac{1}{c} \frac{1}{\sqrt{b\left\|\epsilon_{n}\right\|}} \leq \lambda^{K_{n}} \leq c \frac{1}{\sqrt{b\left\|\epsilon_{n}\right\|}} .
$$

Moreover, for the rescaling levels $1 \leq j \leq K_{n}$ in the good region, we have G1. $C_{n}^{r}(j) \cap F_{n}\left(D_{n}\right)=\emptyset$, 
Fig. 7 The good region and the bad region

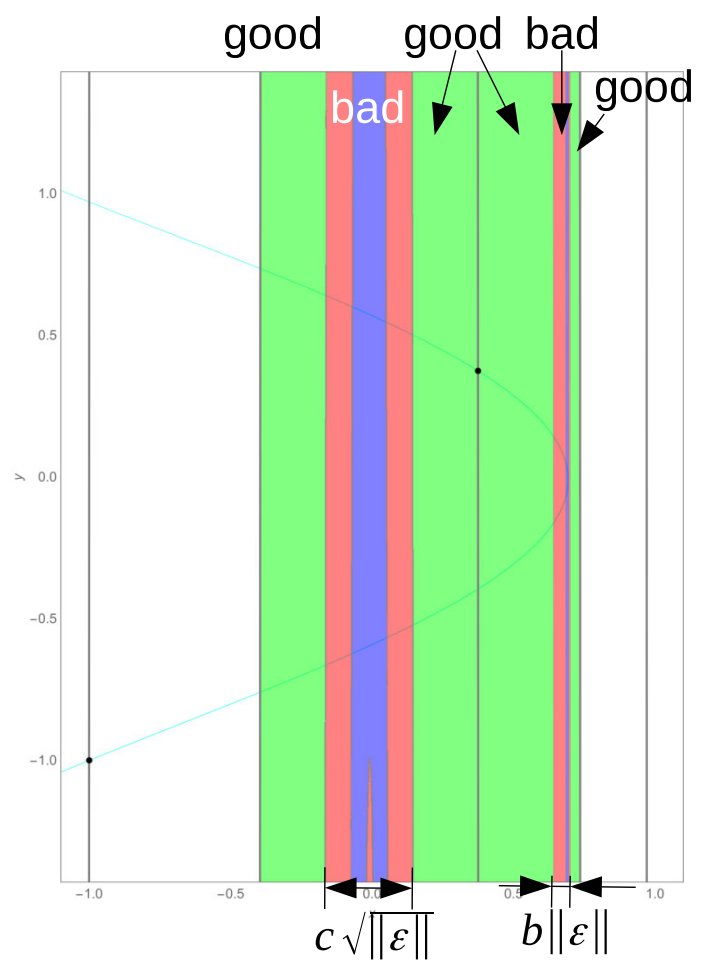

G2. $\left|\pi_{x} z-\pi_{x} \tau_{n}\right|>\frac{b}{c}\left\|\epsilon_{n}\right\|$ for all $z \in C_{n}(j) \cap F_{n}\left(D_{n}\right)$,

G3. $\left|\pi_{x} z-v_{n}\right|>\frac{1}{c} \sqrt{b\left\|\epsilon_{n}\right\|}$ for all $z \in B_{n}(j)$,

G4. $\frac{1}{c}\left(\frac{1}{\lambda}\right)^{2 j}<\left|\pi_{x} z-\pi_{x} \tau_{n}\right|<c\left(\frac{1}{\lambda}\right)^{2 j}$ for all $z \in C_{n}(j) \cap F_{n}\left(D_{n}\right)$, and

G5. $\frac{1}{c}\left(\frac{1}{\lambda}\right)^{j}<\left|\pi_{x} z-v_{n}\right|<c\left(\frac{1}{\lambda}\right)^{j}$ for all $z \in B_{n}(j)$.

For the rescaling levels $j>K_{n}$ in the bad region, we have

B1. $\left|\pi_{x} z-\pi_{x} \tau_{n}\right|<c b\left\|\epsilon_{n}\right\|$ for all $z \in C_{n}(j) \cap F_{n}\left(D_{n}\right)$ and

B2. $\left|\pi_{x} z-v_{n}\right|<c \sqrt{b\left\|\epsilon_{n}\right\|}$ for all $z \in B_{n}(j)$.

First, we estimate the size of the boundary $K_{n}$.

Proof of (8.1) To prove the lower bound, we apply Proposition 5.4 to the definition of $K_{n}$. Assume that $\bar{\epsilon}>0$ is sufficiently small. We have

$$
c\left(\frac{1}{\lambda}\right)^{2\left(K_{n}+1\right)} \leq\left|z_{n}^{(0)}\left(K_{n}+1\right)-\tau_{n}\right| \leq b\left\|\epsilon_{n}\right\| .
$$

for some constant $c>1$. Thus,

$$
\lambda^{K_{n}} \geq \sqrt{\frac{c}{b \lambda^{2}}} \frac{1}{\sqrt{\left\|\epsilon_{n}\right\|}} .
$$


The proof for the upper bound is similar.

To prove the geometric properties, the strategy is to first estimate the distance from the local stable manifold $W_{n}^{t}(j)$ to the tip $\tau_{n}$. Since the rescaling level $C_{n}(j)$ is the union of two components, one is bounded between $W_{n}^{0}(j-1)$ and $W_{n}^{0}(j)$ and the other is bounded between $W_{n}^{2}(j)$ and $W_{n}^{2}(j-1)$, the $x$ coordinate of a point in the level can be estimated by the local stable manifolds.

Claim The inequality

$$
\frac{1}{c}\left(\frac{1}{\lambda}\right)^{2 j} \leq\left|\pi_{x} z-\pi_{x} \tau_{n}\right| \leq c\left(\frac{1}{\lambda}\right)^{2 j}
$$

holds for all $z \in W_{n}^{t}(j) \cap\left(I^{h} \times I^{h}\right)$ with $t \in\{0,2\}, 0 \leq j \leq K_{n}$, and $n \geq 0$.

Proof Assume that $\bar{\epsilon}>0$ is sufficiently small. We only prove the lower bound holds for the case $t=0$. The upper bound and the other case $t=2$ are similar.

To prove the lower bound, we apply Proposition 5.4. Let $z \in W_{n}^{0}(j) \cap$ $\left(I^{h} \times I^{h}\right)$. Then

$$
\begin{aligned}
\left|\pi_{x} z-\pi_{x} \tau_{n}\right| & \geq\left|z_{n}^{(0)}(j)-\tau_{n}\right|-\left|\pi_{x} z-\pi_{x} z_{n}^{(0)}(j)\right| \\
& \geq \frac{1}{c}\left(\frac{1}{\lambda}\right)^{2 j}-c\left\|\epsilon_{n}\right\|\left|I^{h}\right| \\
& \geq\left(\frac{1}{c}-c\left|I^{h}\right|\left\|\epsilon_{n}\right\| \lambda^{2 K_{n}}\right)\left(\frac{1}{\lambda}\right)^{2 j}
\end{aligned}
$$

for some constant $c>1$. By (8.1), there exists $c^{\prime}>1$ such that

$$
\left|\pi_{x} z-\pi_{x} \tau_{n}\right| \geq\left(\frac{1}{c}-\frac{c c^{\prime 2}\left|I^{h}\right|}{b}\right)\left(\frac{1}{\lambda}\right)^{2 j} \geq \frac{1}{2 c}\left(\frac{1}{\lambda}\right)^{2 j}
$$

whenever $b \geq 2 c^{2} c^{\prime 2}\left|I^{h}\right|$.

We prove the first property of the good region.

Property G1 $C_{n}^{r}(j) \cap F_{n}\left(D_{n}\right)=\emptyset$.

Proof Since the right component of the good region $\overline{\bigcup_{j=1}^{K_{n}} C_{n}^{r}(j)}$ is the set bounded between the local manifolds $W_{n}^{2}\left(K_{n}\right)$ and $W_{n}^{2}(0)$, it suffices to show that $W_{n}^{2}\left(K_{n}\right)$ is far away form the image. Compute

$$
f_{n}\left(v_{n}\right)-\left\|\epsilon_{n}\right\| \leq \sup _{z^{\prime} \in D_{n}} h_{n}\left(z^{\prime}\right)=\sup _{z^{\prime} \in D_{n}}\left(f_{n}\left(\pi_{x} z^{\prime}\right)+\epsilon_{n}\left(z^{\prime}\right)\right) \leq f_{n}\left(v_{n}\right)+\left\|\epsilon_{n}\right\| .
$$


By Proposition 5.10, (8.1), and (8.2), there exist constants $c>0$ and $a>1$ such that

$$
\begin{aligned}
\pi_{x} z-\sup _{z^{\prime} \in D_{n}} h_{n}\left(z^{\prime}\right) & \geq\left(\pi_{x} z-\pi_{x} \tau_{n}\right)-\left|\pi_{x} \tau_{n}-f_{n}\left(v_{n}\right)\right|-\left|f_{n}\left(v_{n}\right)-\sup _{z^{\prime} \in D_{n}} h_{n}\left(z^{\prime}\right)\right| \\
& \geq \frac{1}{a}\left(\frac{1}{\lambda}\right)^{2 K_{n}}-c\left\|\epsilon_{n}\right\|-\left\|\epsilon_{n}\right\| \geq\left(\frac{b}{a^{3}}-c-1\right)\left\|\epsilon_{n}\right\|
\end{aligned}
$$

for all $z \in W_{n}^{2}\left(K_{n}\right) \cap\left(I^{h} \times I^{h}\right)$. The coefficient on the right-hand side is positive when $b>0$ is large enough. Consequently, $C_{n}^{r}(j) \cap F_{n}\left(D_{n}\right)=\emptyset$ for all $1 \leq j \leq K_{n}$.

$G 2$ shows that the good region is $\left\|\epsilon_{n}\right\|$ away from the tip.

Property $G 2\left|\pi_{x} z-\pi_{x} \tau_{n}\right|>c b\left\|\epsilon_{n}\right\|$ for all $z \in C_{n}(j) \cap F_{n}\left(D_{n}\right)$.

Proof It is enough to consider only points in the left component $C_{n}^{l}(j)$ because of $G 1$. The left component is the set bounded between $W_{n}^{0}(0)$ and $W_{n}^{0}\left(K_{n}\right)$. Thus, the estimate follows from (8.1) and (8.2).

$G 3$ is an analogue of $G 2$ in $B$.

Property $G 3\left|\pi_{x} z-v_{n}\right| \geq c \sqrt{b\left\|\epsilon_{n}\right\|}$ for all $z \in B_{n}(j)$.

Proof Assume that $z \in B_{n}(j)$ with $1 \leq j \leq K_{n}$ and $n \geq 0$. By Proposition 5.10 and $G 2$, we get

$$
\begin{aligned}
\left|f_{n}\left(\pi_{x} z\right)-f_{n}\left(v_{n}\right)\right| & \geq\left|h_{n}(z)-\pi_{x} \tau_{n}\right|-\left|f_{n}\left(\pi_{x} z\right)-h_{n}(z)\right|-\left|\pi_{x} \tau_{n}-f_{n}\left(v_{n}\right)\right| \\
& \geq\left(c b-1-c^{\prime}\right)\left\|\epsilon_{n}\right\|>\frac{c b}{2}\left\|\epsilon_{n}\right\|
\end{aligned}
$$

for some $c>0$ and $c^{\prime}>0$ when $b>2\left(1+c^{\prime}\right) / c$.

Moreover, by Lemma 5.5, there exists a constant $a>1$ such that

$$
\left|f_{n}\left(\pi_{x} z\right)-f_{n}\left(v_{n}\right)\right| \leq \frac{a}{2}\left(\pi_{x} z-v_{n}\right)^{2}
$$

for all $n \geq 0$ when $\bar{\epsilon}>0$ is small enough. Therefore, $\left|\pi_{x} z-v_{n}\right| \geq$ $\sqrt{\frac{c}{a}} \sqrt{b\left\|\epsilon_{n}\right\|}$.

$G 4$ can be proved by applying (8.2) to the boundary separators of a rescaling level in the good region. $B 1$ follows from (8.1) and (8.2). The proofs of $G 5$ and $B 2$ are similar to the proof of $G 3$. This completes the proof of Proposition 8.2. 


\section{The good region and the expansion estimate}

In this section, we generalize the expansion estimate (7.1) from unimodal maps to Hénon-like maps: the horizontal sizes expand at a definite rate when the elements in a closest approach stay in the good regions (Proposition 9.11). From now on, we fix $b>0$ to be a large constant so that Proposition 8.2 holds and the boundaries between the regions $\left\{K_{n}\right\}_{n=0}^{\infty}$ depend only on $F$.

Assume that $J \subset A_{n} \cup B_{n}$ is a wandering domain in the good region. We split the proof of the expansion estimate (Proposition 9.11) into three cases:

1. $J \subset A_{n}$ (Sect. 9.1),

2. $J \subset B_{n}(j)$ with $1 \leq j<\bar{K}$ for some positive integer $\bar{K}$ (Sect. 9.3), and

3. $J \subset B_{n}$ (j) with $\bar{K} \leq j \leq K_{n}$ (Sect. 9.2).

We will prove Case 3 before Case 2 because the constant $\bar{K}$ has to be determined in Case 3 first.

It is not surprising that the estimate holds when $J$ is far away from the bad region (Cases 1 and 2) because the Hénon-like map $F$ inherits analytical properties from the limiting map $g$ when $F$ is close to $g$. On the other hand, the expansion estimate breaks down in the bad region as explained in the previous section. The intermediate region (Case 3) turns out to be the nontrivial part of the proof because the rescaling trick (Proposition 7.3) does not apply to the Hénon case. The proof depends on the properties of the good region (Proposition 8.2: $G 3$ and $G 5$ ) and relies on the estimations of the contraction of a iteration (Lemma 9.4) and the expansion of rescalings (Lemma 9.5).

To prove that the horizontal size expands, we introduce a technical condition.

Definition 9.1 Let $R>0$. A set $U \subset D(F)$ is $R$-regular if

$$
\frac{v(U)}{l(U)} \leq R \frac{1}{\|\epsilon\|^{1 / 4}} .
$$

In terms of the vertical line argument (Fig. 6), this ensures that the line connecting a pair of horizontal endpoints is far from being parallel to the preimage of a vertical line. We note that the lemmas in Sects. 9.1-9.3 hold for all regular sets. The lemmas will be applied to a regular subset of a wandering domain in Proposition 9.11.

\subsection{Case $J \subset A_{n}$}

In this subsection, we study the expansion rate of the horizontal size when a regular set is in $A$. We show that the expansion estimate for $g$ (Proposition 3.6) also applies to Hénon-like maps when $F_{n}$ is close to the degenerate map $G$. 
Lemma 9.2 For all $R>0$, there exist $\bar{\epsilon}=\bar{\epsilon}(R)>0$ and $E>1$ such that the following properties hold for all $F \in \hat{\mathcal{I}}_{\delta}\left(I^{h} \times I^{v}, \bar{\epsilon}\right)$ and $n \geq 0$ :

If $J \subset A_{n}$ is a closed $R$-regular set, then $J^{\prime}=F_{n}(J)$ is an $R$-regular set in $A_{n} \cup W_{n}^{1}(0) \cup B_{n}$ and

$$
l\left(J^{\prime}\right) \geq E l(J) .
$$

Proof Let $\left(x_{1}, y_{1}\right),\left(x_{2}, y_{2}\right) \in J$ be a pair of horizontal endpoints. Then $l(J)=$ $\left|x_{2}-x_{1}\right|$ and $v(J) \geq\left|y_{2}-y_{1}\right|$. By the mean value theorem, we have

$$
\begin{aligned}
l\left(J^{\prime}\right) & \geq\left|\pi_{x}\left[F_{n}\left(x_{2}, y_{2}\right)-F_{n}\left(x_{1}, y_{2}\right)\right]\right|-\left|\pi_{x}\left[F_{n}\left(x_{1}, y_{2}\right)-F_{n}\left(x_{1}, y_{1}\right)\right]\right| \\
& \geq\left|\frac{\partial h_{n}}{\partial x}\left(\xi, y_{2}\right)\right| l(J)-\left|\frac{\partial \epsilon_{n}}{\partial y}\left(x_{1}, \eta\right)\right| v(J)
\end{aligned}
$$

for some $\xi \in\left(x_{1}, x_{2}\right)$ and $\eta \in\left(y_{1}, y_{2}\right)$. By Lemmas 4.21 and 5.6, we get

$$
l\left(J^{\prime}\right) \geq E l(J)-c\left\|\epsilon_{n}\right\| v(J) \geq\left(E-c R\left\|\epsilon_{n}\right\|^{3 / 4}\right) l(J) \geq \sqrt{E} l(J)
$$

for some constant $c=c(\delta)>0$ when $\bar{\epsilon}=\bar{\epsilon}(R)>0$ is small. This proves (9.2).

Moreover, by (9.3) and $v\left(J^{\prime}\right)=l(J)$, we have

$$
\frac{v\left(J^{\prime}\right)}{l\left(J^{\prime}\right)} \leq \frac{1}{\sqrt{E}} \leq R\left\|\epsilon_{n}\right\|^{-1 / 4}
$$

when $\bar{\epsilon}=\bar{\epsilon}(R)>0$ is small enough. This proves that $J^{\prime}$ is $R$-regular.

\subsection{Case $J \subset B_{n}(k), \bar{K} \leq k \leq K_{n}$}

In this subsection, we prove that the horizontal size expands when a regular set $J$ in the intermediate region of $B$ is first iterated and then rescaled (Lemma 9.3). We first estimate the amount of contraction when the set $J$ is iterated by $F_{n}$ (Lemma 9.4). Then we estimate the size of expansion when the iterated set $F_{n}(J)$ is rescaled by $\Phi_{n}^{k(J)}$ (Lemma 9.5). Finally, we show that the expansion is larger than the contraction:

Lemma 9.3 For all $R>0$, there exist constants $\bar{\epsilon}=\bar{\epsilon}(R)>0, E>1, R^{\prime}>$ 0 , and $c>0$ such that the following properties hold for all $F \in \hat{\mathcal{I}}_{\delta}\left(I^{h} \times I^{v}, \bar{\epsilon}\right)$ and $n \geq 0$ :

If $J \subset B_{n}(k)$ is a closed $R$-regular set and $1 \leq k \leq K_{n}$, then the set $J^{\prime}=\Phi_{n}^{k} \circ F_{n}(J)$ is an $R^{\prime}$-regular set in $C_{n+k}(0)=A_{n+k} \cup W_{n+k}^{1}(0) \cup B_{n+k}$ and

$$
l\left(J^{\prime}\right) \geq c E^{k} l(J) .
$$

The constants $E, R^{\prime}$, and $c$ do not depend on $R$. 
After this subsection, we let $\bar{K}>0$ be a large integer so that (9.4) produces a definite expansion for all $k \geq \bar{K}$. We also let $R=R^{\prime}$ to make the regularity property invariant under the construction of a closest approach.

First, we estimate the amount of contraction when a regular set is iterated.

Lemma 9.4 For all $R>0$, there exist constants $\bar{\epsilon}=\bar{\epsilon}(R)>0, c>1$, and $R^{\prime}>0$ such that the following properties hold for all $F \in \hat{\mathcal{I}}_{\delta}\left(I^{h} \times I^{v}, \bar{\epsilon}\right)$ and $n \geq 0$ :

Suppose that $J \subset B_{n}(k)$ is a closed $R$-regular set and $k \leq K_{n}$. Let $J^{\prime}=$ $F_{n}(J)$. Then

$$
l\left(J^{\prime}\right) \geq c \lambda^{-k} l(J)
$$

and

$$
\frac{v\left(J^{\prime}\right)}{l\left(J^{\prime}\right)} \leq R^{\prime} \frac{1}{\sqrt{\left\|\epsilon_{n}\right\|}}
$$

The constants $c$ and $R^{\prime}$ do not depend on $R$.

Proof Let $\left(x_{1}, y_{1}\right),\left(x_{2}, y_{2}\right) \in J$ be a pair of horizontal endpoints and $x \in\left\{x_{1}, x_{2}\right\}$ be such that $\left|x-v_{n}\right|=\min _{i=1,2}\left|x_{i}-v_{n}\right|$. By the mean value theorem, we have

$$
\begin{aligned}
l\left(J^{\prime}\right) & \geq\left|\pi_{x}\left(F_{n}\left(x_{2}, y_{2}\right)-F_{n}\left(x_{1}, y_{2}\right)\right)\right|-\left|\pi_{x}\left(F_{n}\left(x_{1}, y_{2}\right)-F_{n}\left(x_{1}, y_{1}\right)\right)\right| \\
& \geq\left(\left|f_{n}^{\prime}(\xi)\right|-\left|\frac{\partial \epsilon_{n}}{\partial x}\left(\xi, y_{2}\right)\right|-\left|\frac{\partial \epsilon_{n}}{\partial y}\left(x_{1}, \eta\right)\right| \frac{v(J)}{l(J)}\right) l(J)
\end{aligned}
$$

for some constants $\xi \in\left(x_{1}, x_{2}\right)$ and $\eta \in\left(y_{1}, y_{2}\right)$. Applying Lemma 5.5 to the first term, Lemma 4.21 to the second term, and the definition of a regular set and Lemma 4.21 to the third term, we get

$$
l\left(J^{\prime}\right) \geq\left(\frac{1}{a}\left|x-v_{n}\right|-c_{1}\left(\left\|\epsilon_{n}\right\|^{1 / 2}+R\left\|\epsilon_{n}\right\|^{1 / 4}\right) \sqrt{\left\|\epsilon_{n}\right\|}\right) l(J)
$$

for some constants $a>1$ and $c_{1}>0$. By Proposition 8.2(G3), we have

$$
c_{2}\left|x-v_{n}\right|>\sqrt{\left\|\epsilon_{n}\right\|}
$$

for some constant $c_{2}>1$. We obtain

$$
l\left(J^{\prime}\right) \geq \frac{1}{2 a}\left|x-v_{n}\right| l(J)
$$

when $\bar{\epsilon}=\bar{\epsilon}(R)>0$ is small. Then (9.5) follows from Proposition 8.2(G5). 
Moreover, by (9.7), (9.8), and $v\left(J^{\prime}\right)=l(J)$, we get

$$
\frac{v\left(J^{\prime}\right)}{l\left(J^{\prime}\right)} \leq \frac{2 a}{\left|x-v_{n}\right|} \leq R^{\prime} \frac{1}{\sqrt{\left\|\epsilon_{n}\right\|}}
$$

where $R^{\prime}=2 a c_{2}$.

Then we estimate the size of expansion when a wandering domain is rescaled.

Lemma 9.5 For all $R>0$, there exist constants $\bar{\epsilon}=\bar{\epsilon}(R)>0$ and $E>1$ such that the following properties hold for all $F \in \hat{\mathcal{I}}_{\delta}\left(I^{h} \times I^{v}, \bar{\epsilon}\right)$ and $n \geq 0$ : If $J \subset C_{n}(k)$ is a closed set and $\frac{v(J)}{l(J)} \leq R \frac{1}{\sqrt{\left\|\epsilon_{n}\right\|}}$, then

$$
l\left(\Phi_{n}^{j}(J)\right) \geq(\lambda E)^{j} l(J)
$$

and

$$
\frac{v\left(\Phi_{n}^{j}(J)\right)}{l\left(\Phi_{n}^{j}(J)\right)} \leq R \frac{1}{\sqrt{\left\|\epsilon_{n}\right\|}}
$$

for all integer $j$ with $0 \leq j \leq k$. The constant $E$ does not depend on $R$.

Proof Let $E^{\prime}>1$ be the expansion factor defined in Lemma 5.6 and $E=\sqrt{E^{\prime}}$. The proof is by induction on $j$. The lemma holds for the case $j=0$ by definition.

Assume that the lemma is true for some integer $j \leq k$. We show that the lemma also holds for $j+1 \leq k$. Let $\left(x_{1}, y_{1}\right),\left(x_{2}, y_{2}\right) \in \Phi_{n}^{j}(J)$ be a pair of horizontal endpoints. By the mean value theorem, we have

$$
\begin{aligned}
l\left(\Phi_{n}^{j+1}(J)\right) \geq & \left|\pi_{x}\left(\phi_{n+j}\left(x_{2}, y_{2}\right)-\phi_{n+j}\left(x_{1}, y_{2}\right)\right)\right| \\
& -\left|\pi_{x}\left(\phi_{n+j}\left(x_{1}, y_{2}\right)-\phi_{n+j}\left(x_{1}, y_{1}\right)\right)\right| \\
\geq & \lambda_{n+j}\left|\frac{\partial h_{n}}{\partial x}\left(\xi_{j}, y_{2}\right)\right| l\left(\Phi_{n}^{j}(J)\right)-\lambda_{n+j}\left|\frac{\partial \epsilon_{n+j}}{\partial y}\left(x_{1}, \eta_{j}\right)\right| v\left(\Phi_{n}^{j}(J)\right)
\end{aligned}
$$

for some $\xi_{j} \in\left(x_{1}, x_{2}\right) \subset I^{A C}$ and $\eta_{j} \in\left(y_{1}, y_{2}\right)$. Then we apply Lemma 5.6 to the first term and $(9.10)$ to the second term. We get

$$
\begin{aligned}
l\left(\Phi_{n}^{j+1}(J)\right) & \geq \lambda_{n+j}\left(E^{\prime}-\frac{R}{\delta} \sqrt{\left\|\epsilon_{n}\right\|}\right) l\left(\Phi_{n}^{j}(J)\right) \\
& \geq \lambda \operatorname{El}\left(\Phi_{n}^{j}(J)\right)
\end{aligned}
$$

when $\bar{\epsilon}>0$ is small. Then (9.9) follows from the induction hypothesis. 
Moreover, the vertical sizes are related by $v\left(\Phi_{n}^{j+1}(J)\right)=\lambda_{n+j} v\left(\Phi_{n}^{j}(J)\right)$. By (9.11) and the induction hypothesis, we get

$$
\frac{v\left(\Phi_{n}^{j+1}(J)\right)}{l\left(\Phi_{n}^{j+1}(J)\right)} \leq \frac{1}{E^{\prime}-\frac{1}{\delta} R \sqrt{\left\|\epsilon_{n}\right\|}} \frac{v\left(\Phi_{n}^{j}(J)\right)}{l\left(\Phi_{n}^{j}(J)\right)}<R \frac{1}{\sqrt{\left\|\epsilon_{n}\right\|}}
$$

when $\bar{\epsilon}>0$ is small.

Therefore, the lemma is proved by induction.

Finally, we can now prove the expansion estimate for the intermediate region.

Proof of Lemma 9.3 The expansion estimate (9.4) follows from (9.5) and (9.9).

To show that $J^{\prime}$ is a regular set, we apply (9.6), (9.10), and Proposition 4.16. Then

$$
\frac{v\left(J^{\prime}\right)}{l\left(J^{\prime}\right)} \leq R^{\prime} \frac{1}{\sqrt{\left\|\epsilon_{n}\right\|}} \leq R^{\prime} c \frac{1}{\left\|\epsilon_{n+1}\right\|^{1 / 4}} \leq R^{\prime} c \frac{1}{\left\|\epsilon_{n+k}\right\|^{1 / 4}}
$$

where $c>0$ is a constant. Therefore, the set $J^{\prime}$ is $R^{\prime} c$-regular.

\subsection{Case $J \subset B_{n}(k), 1 \leq k<\bar{K}$}

In this subsection, we prove the expansion estimate holds when the wandering domain is inside $B$ but far away from the bad region. Although the rescaling trick does not work in the nondegenerate case, we still can apply it to the limiting degenerate Hénon-like map $G$ to prove the expansion estimate for $G$. Then we show that the estimate can be promoted to Hénon-like maps that are close to $G$ because of continuity and $\bar{K}$ is a fixed number.

In the limiting case, we have

$$
\lim _{n \rightarrow \infty} F_{n}(x, y)=(g(x), x) \text { and } \lim _{n \rightarrow \infty} \phi_{n}(x, y)=(-\lambda)(g(x), y) .
$$

Then we get

$$
\lim _{n \rightarrow \infty} \Phi_{n}^{j}(x, y)=\left([(-\lambda) g]^{j}(x),(-\lambda)^{j} y\right)
$$

and

$$
\lim _{n \rightarrow \infty} \Phi_{n}^{j} \circ F_{n}(x, y)=\left([(-\lambda) g]^{j} \circ g(x),(-\lambda)^{j} x\right) .
$$

The expression $[(-\lambda) g]^{j}$ means the $j$-fold iterate of the map $x \rightarrow(-\lambda) g(x)$. 
The next lemma is a version of the rescaling trick (Lemma 7.3) in the limiting case. Let $q^{l}(j)=-\left(\frac{1}{\lambda}\right)^{j+1}$ and $q^{r}(j)=\left(\frac{1}{\lambda}\right)^{j+1}$. Then the rescaling levels of the unimodal map $g$ have the form $B_{g}(j)=\left(q^{l}(j-1), q^{l}(j)\right) \cup$ $\left(q^{r}(j), q^{r}(j-1)\right)$.

Lemma 9.6 (The rescaling trick) Assume that $j \geq 0$ is an integer. Then

$$
[(-\lambda) g]^{j} \circ g(x)=g\left((-\lambda)^{j} x\right)
$$

for all $x$ with $q^{l}(j-1) \leq x \leq q^{r}(j-1)$.

Proof The lemma follows either from the functional equation (3.1) or Proposition 7.3.

Then we estimate the size of expansion in each rescaling level.

Lemma 9.7 There exist constants $E, E^{\prime}>1$ such that

$$
E \lambda^{j} \leq\left|\frac{\mathrm{d}[(-\lambda) g]^{j} \circ g}{\mathrm{~d} x}(x)\right| \leq E^{\prime} \lambda^{j}
$$

for all $x \in B_{g}(j)$ and $j \geq 0$.

Proof By the rescaling trick and the chain rule, we have

$$
\frac{\mathrm{d}[(-\lambda) g]^{j} \circ g}{\mathrm{~d} x}(x)=(-\lambda)^{j} g^{\prime}\left((-\lambda)^{j} x\right)
$$

for all $|x| \leq\left(\frac{1}{\lambda}\right)^{j}$. Then (9.13) follows from Proposition 3.6.

Assume that $J \subset B_{n}(j)$ is a regular set. As usual, we estimate the expansion rate by iterating a pair of horizontal endpoints. In order to apply the mean value theorem to the horizontal endpoints, the map $\Phi_{n}^{j} \circ F_{n}$ has to be defined on a convex (rectangular) neighborhood of $B_{n}(j)$. To extend the expansion estimate to the Hénon-like map $F_{n}$, we also need to show that the partition element $B_{n}(j)$ is close to the partition element of the limiting case $G$ when $\bar{\epsilon}$ is small. This is true because of the hyperbolicity of the saddle points. The technical details are left to the reader.

Lemma 9.8 For all $d>0$ and integer $j \geq 1$, there exist a constant $\bar{\epsilon}=$ $\bar{\epsilon}(d, j)>0$ and two closed intervals $U^{l} \subset\left[q^{l}(j-1)-d, q^{l}(j)+d\right]$ and $U^{r} \subset\left[q^{r}(j)-d, q^{r}(j-1)+d\right]$ such that the following properties hold:

Let $F \in \hat{\mathcal{I}}_{\delta}\left(I^{h} \times I^{v}, \bar{\epsilon}\right)$ and $U=U^{l} \cup U^{r}$. Then for all $n \geq 0$, we have

1. $B_{n}^{l}(j) \subset U^{l} \times I_{n}^{v}, B_{n}^{r}(j) \subset U^{r} \times I_{n}^{v}$, and 
2. the map $\Phi_{n}^{j} \circ F_{n}$ is defined on $U \times I_{n}^{v}$ where $U=U^{l} \cup U^{r}$.

The expansion estimate, Lemma 9.7, also applies to the nondegenerate case.

Lemma 9.9 For all integer $j \geq 1$, there exist constants $\bar{\epsilon}(j)>0, E>1$, and $E^{\prime}>1$ such that for all $F \in \hat{\mathcal{I}}_{\delta}\left(I^{h} \times I^{v}, \bar{\epsilon}\right)$, the estimate

$$
E \lambda^{j} \leq\left|\frac{\partial \pi_{x} \circ \Phi_{n}^{j} \circ F_{n}}{\partial x}(x, y)\right| \leq E^{\prime} \lambda^{j}
$$

holds for all $(x, y) \in U \times I_{n}^{v}$ and $n \geq 0$ where $U$ is the set defined in Lemma 9.8.

Proof By analytic continuation, the map $x \rightarrow[(-\lambda) g]^{j} \circ g(x)$ has an analytic extension to a neighborhood of $B_{g}(j)$. By continuity, the expansion estimate (9.13) (with possibly different constants) holds on some neighborhood of $B_{g}(j)$. That is, there exist $E>1, E^{\prime}>1$, and $d=d(j)>0$ such that

$$
E \lambda^{j} \leq\left|\frac{\mathrm{d}[(-\lambda) g]^{j} \circ g}{\mathrm{~d} x}(x)\right| \leq E^{\prime} \lambda^{j}
$$

for all $x \in\left[q^{l}(j-1)-d, q^{l}(j)+d\right] \cup\left[q^{r}(j)-d, q^{r}(j-1)+d\right]$. Also, the map $\pi_{x} \circ \Phi_{n}^{j} \circ F_{n}$ (and its derivative) depends continuously on the sup-norm $\|F-G\|$. Together with Lemma 9.8, there exist $\bar{\epsilon}=\bar{\epsilon}(j)>0$ and a union $U$ of two closed intervals such that $B_{n}(j) \subset U \times I_{n}^{v}$ and

$$
\sqrt{E} \lambda^{j} \leq\left|\frac{\partial \pi_{x} \circ \Phi_{n}^{j} \circ F_{n}}{\partial x}(x, y)\right| \leq\left(E^{\prime}\right)^{2} \lambda^{j}
$$

for all $F \in \hat{\mathcal{I}}_{\delta}\left(I^{h} \times I^{v}, \bar{\epsilon}\right)$ and $(x, y) \in U \times I_{n}^{v}$.

Finally, we estimate the size of expansion.

Lemma 9.10 For all $\bar{K}>0$ and $R>0$, there exist constants $\bar{\epsilon}=\bar{\epsilon}(\bar{K}, R)>$ 0 and $E>1$ such that the following properties hold for all $F \in \hat{\mathcal{I}}_{\delta}\left(I^{h} \times I^{v}, \bar{\epsilon}\right)$ and $n \geq 0$ :

If $J \subset B_{n}(k)$ is a connected closed $R$-regular set and $k \leq \min \left(\bar{K}, K_{n}\right)$, then $J^{\prime}=\Phi_{n}^{k} \circ F_{n}(J)$ is an $R$-regular set in $C_{n+k}(0)=A_{n+k} \cup W_{n+k}^{1}(0) \cup B_{n+k}$ and

$$
l\left(J^{\prime}\right) \geq E \lambda^{k} l(J) .
$$

Proof Let $\bar{\epsilon}=\bar{\epsilon}(\bar{K})>0$ be sufficiently small that the expansion estimate in Lemma 9.9 holds for all $j \leq \bar{K}$. Let $J \subset B_{n}(k)$ be a connected closed 
$R$-regular set with $k \leq \min \left(\bar{K}, K_{n}\right)$ and $n \geq 0$. Also, set $G=\Phi_{n}^{k} \circ F_{n}$ and $G_{x}=\pi_{x} \circ G$. Then $J^{\prime}=G(J)$. We prove the lemma for the case of $J \subset B_{n}^{l}(k)$. The other case $J \subset B_{n}^{r}(k)$ is similar.

Let $\left(x_{1}, y_{1}\right),\left(x_{2}, y_{2}\right) \in J$ be a pair of horizontal endpoints. By the mean value theorem, we have

$$
\begin{aligned}
l\left(J^{\prime}\right) & \geq\left|G_{x}\left(x_{2}, y_{2}\right)-G_{x}\left(x_{1}, y_{2}\right)\right|-\left|G_{x}\left(x_{1}, y_{2}\right)-G_{x}\left(x_{1}, y_{1}\right)\right| \\
& \geq\left|\frac{\partial G_{x}}{\partial x}\left(\xi, y_{2}\right)\right| l(J)-\left|\frac{\partial G_{x}}{\partial y}\left(x_{1}, \eta\right)\right| v(J) \\
& \geq\left(\left|\frac{\partial G_{x}}{\partial x}\left(\xi, y_{2}\right)\right|-\left|\frac{\partial G_{x}}{\partial y}\left(x_{1}, \eta\right)\right| R\left\|\epsilon_{n}\right\|^{-1 / 4}\right) l(J)
\end{aligned}
$$

for some $\xi \in\left(x_{1}, x_{2}\right)$ and $\eta \in\left(y_{1}, y_{2}\right)$.

The first term can be estimated by Lemma 9.9. To bound the second term $\frac{\partial G_{x}}{\partial y}\left(x_{1}, \eta\right)$, we compute

$$
\frac{\partial G_{x}}{\partial y}\left(x_{1}, \eta\right)=\frac{\partial \pi_{x} \circ \Phi_{n}^{k}}{\partial x} \circ F_{n}\left(x_{1}, \eta\right) \frac{\partial \epsilon_{n}}{\partial y}\left(x_{1}, \eta\right) .
$$

By compactness, one can find a constant $c>0$ that bounds $\frac{\partial \pi_{x} \circ \Phi_{n}^{k}}{\partial x}$ for all $k \leq \bar{K}$ and all Hénon-like maps that are close to the limiting map $G$. Then

$$
\left|\frac{\partial G_{x}}{\partial y}\left(x_{1}, \eta\right)\right| \leq c c^{\prime}\left\|\epsilon_{n}\right\|
$$

for some $c^{\prime}>0$ by (2.1). The inequality (9.15) becomes

$$
l\left(J^{\prime}\right) \geq E \lambda^{k}\left(1-\frac{c c^{\prime}}{E \lambda^{k}} R\left\|\epsilon_{n}\right\|^{3 / 4}\right) l(J) \geq \sqrt{E} \lambda^{k} l(J)
$$

for some constant $E>1$ when $\bar{\epsilon}=\bar{\epsilon}(R)>0$ is small.

To prove that $J^{\prime}$ is $R$-regular, we use (9.16) and $v\left(J^{\prime}\right)=\left(\prod_{j=0}^{k(J)-1} \lambda_{j+n}\right) l(J)$. Assume that $\bar{\epsilon}=\bar{\epsilon}(\bar{K})$ is small enough that $\prod_{j=0}^{i-1} \lambda_{j+n} \leq 2 \lambda^{i}$ for all $1 \leq i \leq \bar{K}$ and $n \geq 0$. Then

$$
\frac{v\left(J^{\prime}\right)}{l\left(J^{\prime}\right)} \leq \frac{2 \lambda^{k} l(J)}{\sqrt{E} \lambda^{k} l(J)}=\frac{2}{\sqrt{E}} \leq R\left\|\epsilon_{n+k}\right\|^{-1 / 4}
$$

when $\bar{\epsilon}=\bar{\epsilon}(R)$ is small enough. 


\subsection{The expansion estimate}

Finally, we apply the lemmas to a regular subset of a wandering domain in the good region to prove the expansion estimate.

Proposition 9.11 There exist constants $\bar{\epsilon}>0, E>1$, and $R>0$ such that the following properties hold for all $F \in \hat{\mathcal{I}}_{\delta}\left(I^{h} \times I^{v}, \bar{\epsilon}\right)$ :

Suppose that $J \subset A \cup B$ is a connected closed $R$-regular subset of a wandering domain of $F$ and $\left\{J_{n}\right\}_{n=0}^{\infty}$ is the $J$-closest approach. If $k_{n} \leq K_{r(n)}$ for all $n \leq m$, then $J_{n}$ is $R$-regular for all $n \leq m+1$ and

$$
l_{n+1} \geq E l_{n}
$$

for all $n \leq m$.

Proof Let $R>0$ be the constant from Lemma 9.3 that makes the regularity property invariant under the construction of a closest approach. Also, let $\bar{K}>0$ be a large number that $(9.5)$ produces a strict expansion for all $k>\bar{K}$. Then the expansion estimate (9.17) follows from Lemmas 9.2, 9.10, and 9.3.

\section{The bad region and the thickness}

So far we have encountered two problems from the occurrence of the bad regions. First, the horizontal size contracts when an element in a closest approach enters a bad region. Second, the contraction rate does not have a uniform bound. In this section, we will resolve the problems and then prove the main theorem (Theorem 10.14).

First, we introduce the quantity, called "thickness" (Definition 10.1), which gives a good approximation of the horizontal size when the expansion estimate breaks down. We show that the contraction of thicknesses is controlled by the Jacobian of the map (Proposition 10.5). Then, we study the relationship between the horizontal sizes and the thicknesses by defining a double sequence (Definition 10.2). We obtain two inequalities to keep track of every horizontal size in a closest approach (Proposition 10.9). Finally, we show that the closest approach can enter bad regions at most finitely many times (Proposition 10.13). This is achieved by first proving the Two-Row Lemma (Lemma 10.11) and then applying the lemma inductively (Lemma 10.12). Therefore, we may assume without lose of generality that a closest approach stays in the good regions forever by starting from the tail of a rescaled orbit and then prove the main theorem. 
Fig. 8 The horizontal size $l$, the vertical size $v$, and the thickness $w$ of a set $J$. In this picture, $S$ is a largest square subset of $J$

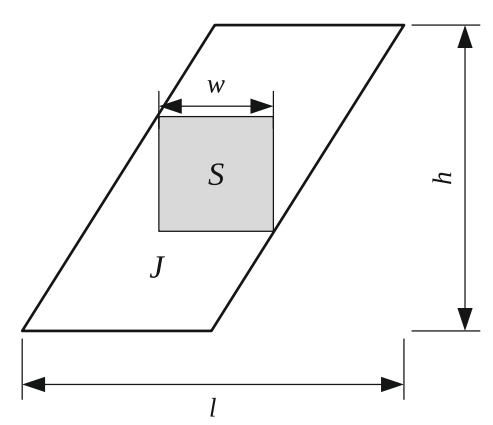

\subsection{The thickness and the largest square subset}

In Sect. 8, we showed that the expansion estimate breaks down in the bad region by the vertical line argument (Fig. 6). When a wandering domain is iterated in the bad region, the shape of the image turns vertically (Fig. 6b) and thus the iteration of the horizontal endpoints cannot provide a good estimate of the horizontal size. On the other hand, unlike the one dimensional case, a wandering domain has area (Fig. 6c). The length of a horizontal crosssection dominates the horizontal size. In this subsection, we introduce the "thickness" to quantify the length of a horizontal cross-section and then study the contraction rate of the thicknesses in a closest approach.

Definition 10.1 A set $S \subset \mathbb{R}^{2}$ is a square if $S=\left[x_{1}, x_{2}\right] \times\left[y_{1}, y_{2}\right]$ and $\left|x_{2}-x_{1}\right|=\left|y_{2}-y_{1}\right|$. The thickness of a set $J \subset \mathbb{R}^{2}$ is the quantity $w(J)=$ $\sup \{l(S)\}$ where the supremum is evaluated over all square subsets $S \subset J$. A largest square subset is a square subset $S \subset J$ such that $l(S)=w(J)$. Figure 8 shows a comparison between the sizes of a set.

Next, we show that the contraction rate of the thicknesses is the Jacobian of the map.

Lemma 10.2 There exist constants $\bar{\epsilon}>0$ and $c>0$ such that the following property holds for all $F \in \hat{\mathcal{I}}_{\delta}\left(I^{h} \times I^{v}, \bar{\epsilon}\right)$ and $n \geq 0$ :

If $S \subset D_{n}$ is a square, then there exists a square $S^{\prime} \subset F_{n}(S)$ such that

$$
l\left(S^{\prime}\right) \geq c \frac{\left\|\epsilon_{n}\right\|}{\left|I_{n}^{v}\right|} l(S)
$$

Proof The lemma is trivial when $F$ is degenerate. We assume that $F$ is nondegenerate.

Let $S=[x, a] \times\left[y_{1}, y_{2}\right],\left(x_{1}^{\prime}, x\right)=F_{n}\left(x, y_{2}\right),\left(x_{2}^{\prime}, x\right)=F_{n}\left(x, y_{1}\right)$, and $W=b\left(x_{2}^{\prime}-x_{1}^{\prime}\right)>0$ where $b>0$ is a constant. Define $x^{\prime}=\frac{x_{1}^{\prime}+x_{2}^{\prime}}{2}$ and $S^{\prime}=\left[x^{\prime}-\frac{1}{2} W, x^{\prime}+\frac{1}{2} W\right] \times[x, x+W]$. We claim that $S^{\prime} \subset F_{n}(S)$ for some uniform constant $b$. 


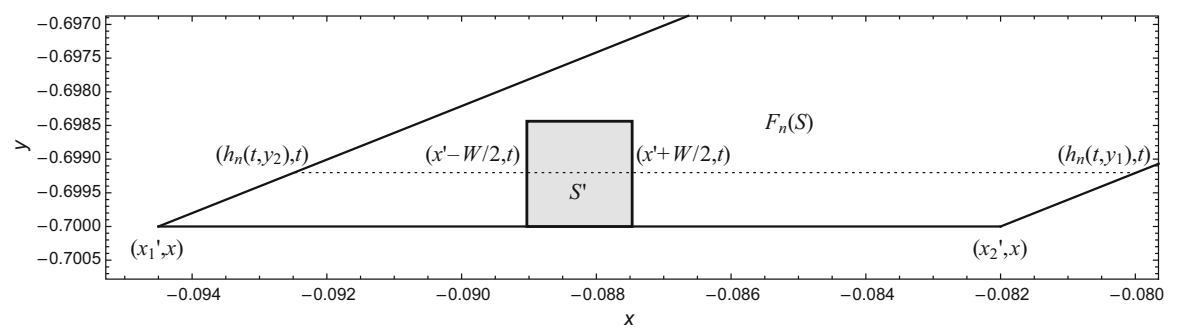

Fig. 9 Four points on the cross section $y=t$

If the claim is true, by the mean value theorem, we have $l\left(S^{\prime}\right)=W=$ $b \frac{\partial \epsilon_{n}}{\partial y}(x, \eta) l(S)$ for some $\eta \in\left(y_{1}, y_{2}\right)$. Then $l\left(S^{\prime}\right) \geq \frac{b c}{\left|I_{n}^{v}\right|}\left\|\epsilon_{n}\right\| l(S)$ for some constant $c>0$ by (4.2) (the definition of $\hat{\mathcal{I}}$ ). This proves (10.1).

Proving the claim is equivalent to show that the inequality

$$
h_{n}\left(t, y_{2}\right)<x^{\prime}-\frac{1}{2} W<x^{\prime}+\frac{1}{2} W<h_{n}\left(t, y_{1}\right)
$$

holds for all $t \in[x, x+W]$. The four terms correspond to the intersection of the boundaries of $F_{n}(S)$ and $S^{\prime}$ with the horizontal cross section $y=t$. See Fig. 9 for an illustration. We only prove the first half $h_{n}\left(t, y_{2}\right)<x^{\prime}-\frac{1}{2} W$ of (10.2) and the second half is similar. By the mean value theorem and the compactness of the domain, there exist $\xi \in(x, t)$ and $E>1$ such that

$$
\left|h_{n}\left(t, y_{2}\right)-x_{1}^{\prime}\right|=\left|h_{n}\left(t, y_{2}\right)-h_{n}\left(x, y_{2}\right)\right|=\left|\frac{\partial h_{n}}{\partial x}\left(\xi, y_{2}\right)\right||t-x| \leq E W .
$$

We get

$$
\begin{aligned}
& \left(x^{\prime}-\frac{1}{2} W\right)-h_{n}\left(t, y_{2}\right)=\left[\left(x^{\prime}-\frac{1}{2} W\right)-x_{1}^{\prime}\right]-\left[h_{n}\left(t, y_{2}\right)-x_{1}^{\prime}\right] \\
& \geq\left(\frac{x_{2}^{\prime}-x_{1}^{\prime}}{2}-\frac{1}{2} W\right)-E W=\left[\frac{1}{2}-\left(\frac{1}{2}+E\right) b\right]\left(x_{2}^{\prime}-x_{1}^{\prime}\right)>0
\end{aligned}
$$

when $b<\frac{1}{1+2 E}$. Therefore, the first half of the inequality is proved.

Remark 10.3 The lemma depends on the universality around the tip (Proposition 4.16 item 5, [11, Theorem 7.9]). The property provides a uniform lower bound for the Jacobian. It ensures that the thickness (area) does not contract too fast.

Finally, we estimate the rate of expansion when a wandering domain is rescaled. Recall that $\lambda_{n}=\left|s_{n}^{\prime}(x)\right|$ is the rescaling factor of the affine rescaling map $s_{n}$. 


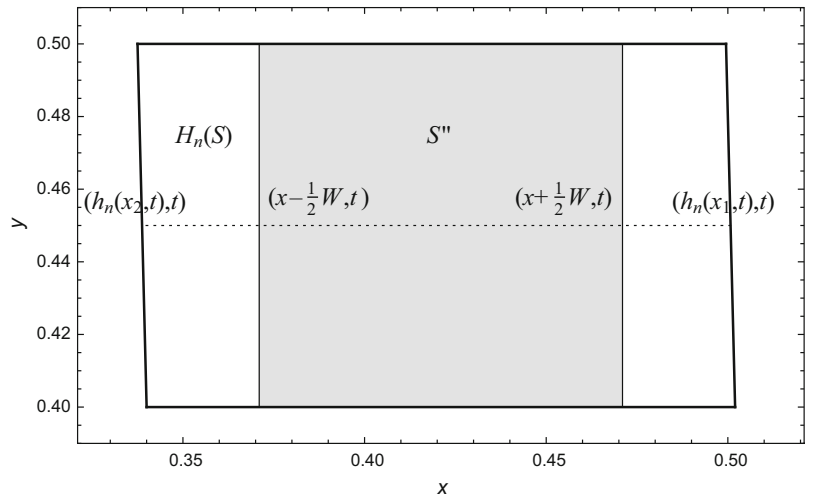

Fig. 10 Four points on the cross section $y=t$

Lemma 10.4 There exists $\bar{\epsilon}>0$ such that the following property holds for all $F \in \hat{\mathcal{I}}_{\delta}\left(I^{h} \times I^{v}, \bar{\epsilon}\right)$ and $n \geq 0$ :

If $S \subset C_{n}$ is a square, then there exists a square $S^{\prime} \subset \phi_{n}(S)$ such that

$$
l\left(S^{\prime}\right)=\lambda_{n} l(S) .
$$

Proof Let $S=\left[x_{1}, x_{2}\right] \times\left[y_{1}, y_{2}\right], W=l(S), x=\frac{1}{2}\left[h_{n}\left(x_{2}, y_{1}\right)+h_{n}\left(x_{1}, y_{1}\right)\right]$, and $S^{\prime \prime}=\left[x-\frac{1}{2} W, x+\frac{1}{2} W\right] \times\left[y_{1}, y_{2}\right]$. Then $S^{\prime \prime}$ is a square with $l\left(S^{\prime \prime}\right)=l(S)$.

The idea is to show that $S^{\prime \prime} \subset H_{n}(S)$ by proving the inequality

$$
h_{n}\left(x_{2}, t\right)<x-\frac{1}{2} W<x+\frac{1}{2} W<h_{n}\left(x_{1}, t\right)
$$

holds for all $t \in\left[y_{1}, y_{2}\right]$. The four terms in the inequality are four points on the horizontal cross section $y=t$. See Fig. 10 for an illustration. The proof is similar to Lemma 10.2 and is left to the reader.

Finally, let $S^{\prime}=\Lambda_{n}\left(S^{\prime \prime}\right)$. Then $S^{\prime}$ satisfies the desired properties.

The convention of the subscript will be applied also to the thickness $w_{n}=$ $w\left(J_{n}\right)$. Recall from Definition 6.5 that $r(n)$ is the renormalization scale of the element $J_{n}$ in the closest approach, i.e. $J_{n}$ belongs to the domain of $F_{r(n)} \equiv$ $R^{r(n)} F$. By Lemmas 10.2 and 10.4, the contraction rate of the thicknesses is estimated by the following proposition.

Proposition 10.5 There exist constants $\bar{\epsilon}>0$ and $c>0$ such that the following property holds for all $F \in \hat{\mathcal{I}}_{\delta}\left(I^{h} \times I^{v}, \bar{\epsilon}\right)$ :

If $J \subset A \cup B$ is a compact subset of a wandering domain of $F$ and $\left\{J_{n}\right\}_{n=0}^{\infty}$ is the $J$-closest approach, then

$$
w_{n+1} \geq c \frac{\left\|\epsilon_{r(n)}\right\|}{\left|I_{r(n)}^{v}\right|} w_{n}
$$


for all $n \geq 0$.

Since $\left\|\epsilon_{n}\right\|$ decreases super-exponentially and $\left|I_{n}^{v}\right|$ increases exponentially as $n \rightarrow \infty$, we can simplify the estimate as follows.

Corollary 10.6 There exist a constant $\bar{\epsilon}>0$ such that the following property holds for all $F \in \hat{\mathcal{I}}_{\delta}\left(I^{h} \times I^{v}, \bar{\epsilon}\right)$ :

If $J \subset A \cup B$ is a compact subset of a wandering domain of $F$ and $\left\{J_{n}\right\}_{n=0}^{\infty}$ is the $J$-closest approach, then

$$
w_{n+1} \geq\left\|\epsilon_{r(n)}\right\|^{2} w_{n}
$$

for all $n \geq 0$.

\subsection{The double sequence}

In this subsection, we study the relationships between the horizontal sizes and the thicknesses in a closest approach. We define a sequence with two indices, called a double sequence. A double sequence consists of rows. Each row is a closest approach (Definition 6.5) and has an element in the bad region.

Definition 10.7 Let $\delta>0$ and $I^{v} \supset I^{h} \ni I$. Assume that $\bar{\epsilon}>0$ is small and $F \in \hat{\mathcal{I}}_{\delta}\left(I^{h} \times I^{v}, \bar{\epsilon}\right)$ is a nondegenerate open map.

Suppose that $J \subset A \cup B$ is a square subset of a wandering domain of $F$. We define $\left\{J_{n}^{(j)}\right\}_{n \geq 0,0 \leq j \leq \bar{j}}$ and $\left\{F_{n}^{(j)}\right\}_{n \geq 0,0 \leq j \leq \bar{j}}$ by induction where $\bar{j} \in$ $\mathbb{N} \cup\{0, \infty\}:$

1. For $j=0$, let $J_{0}^{(0)}=J$ and $F_{0}^{(0)}=F$.

2. Assume that $J_{0}^{(j)} \subset A\left(F_{0}^{(j)}\right) \cup B\left(F_{0}^{(j)}\right)$ is a square subset of a wandering domain of $F_{0}^{(j)}$. Let $\left\{J_{n}^{(j)}\right\}_{n=0}^{\infty}$ be a $J_{0}^{(j)}$-closest approach (Definition 6.5).

(a) If an element $J_{n}^{(j)}$ enters the bad region, i.e. $k_{n}^{(j)}>K_{r^{(j)}(n)}^{(j)}$ for some $n \geq 0$, let $N$ be the smallest index. The index $n^{(j)} \equiv N$ is called the time span in good regions. Let $J_{0}^{(j+1)}$ be a largest square subset of $J_{N+1}^{(j)}$ and $F_{0}^{(j+1)}=F_{r^{(j)}(N+1)}^{(j)}$.

(b) If the elements stay in the good regions forever, then the construction stops. Let $\bar{j}=j$ and $n^{(j)}=\infty$.

3. If the construction never ends, let $\bar{j}=\infty$.

The sequence $\left\{J_{n}^{(j)}\right\}_{n \geq 0,0 \leq j \leq \bar{j}}$ with two indices is called a double sequence generated by $J$ or a $J$-double sequence. The super-script $j$ is called row. The integer $\bar{j}$ is the total number of rows. Equivalently, the sequence enters bad regions $\bar{j}$ times. Figure 11 shows an illustration of the construction. 


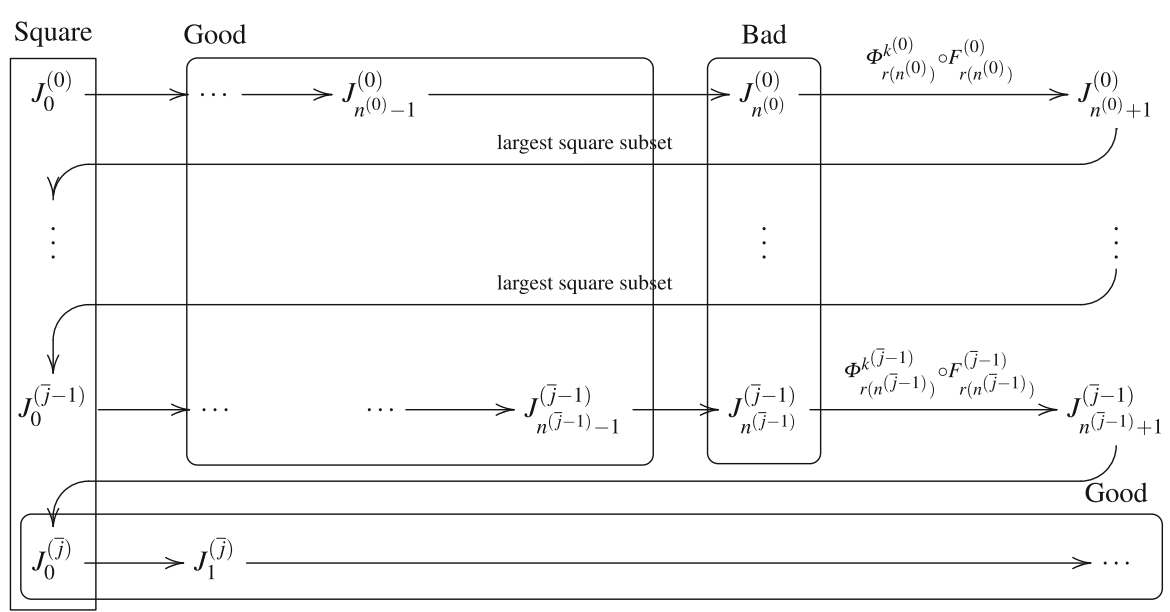

Fig. 11 The construction of a double sequence

To be consistent, the superscript is assigned to the row, and the subscript is assigned to the renormalization scale or the index of an element in a closest approach. For example, the superscript is introduced to the notations: $A_{n}^{(j)}=$ $A\left(F_{n}^{(j)}\right), B_{n}^{(j)}=B\left(F_{n}^{(j)}\right), C_{n}^{(j)}=C\left(F_{n}^{(j)}\right), D_{n}^{(j)}=D\left(F_{n}^{(j)}\right), l_{n}^{(j)}=l\left(J_{n}^{(j)}\right)$, $w_{n}^{(j)}=w\left(J_{n}^{(j)}\right)$, and $k_{n}^{(j)}=k\left(J_{n}^{(j)}\right)$. In the following, we write $r(n)=r^{(j)}(n)$ when the context is clear. For example $F_{r(N+1)}^{(j)}=F_{r^{(j)}(N+1)}^{(j)}$, where $N=n^{(j)}$. Also, let $\epsilon^{(j)}=\epsilon_{r(N)}^{(j)}, K^{(j)}=K_{r(N)}^{(j)}$, and $k^{(j)}=k_{N}^{(j)}$. For convenience, let $m^{(j)}=n^{(j)}+1$.

Example 10.8 Figure 12 shows an example of a double sequence. The map is the same as in Example 6.6. Let $J_{0}^{(0)} \subset A_{0}^{(0)}$ be a square and $r^{(0)}(0)=0$. We construct a closest approach starting from $J_{0}^{(0)}$. According to the figure, $C_{0}^{r}(1)$ has a nonempty intersection with the image $F_{0}\left(D_{0}\right)$. Thus, $K_{r(1)}^{(0)}=K_{0}^{(0)}=0$ and $J_{1}^{(0)} \subset B_{r(1)}(1)$ is in the bad region. Let $n^{(0)}=1$.

Then we start a new row $j=1$ by letting $J_{0}^{(1)}$ be a largest square subset of $J_{2}^{(0)}$ (Fig. 12e). As we can see from the figure, the thickness $w_{2}^{(0)}=l_{0}^{(1)}$ gives a good approximation of the horizontal size $l_{2}^{(0)}$ after the sequence enters the bad region. The construction of rows continues until the sequence stops entering bad regions.

From the definition, we are able to keep track of every horizontal size by using the thickness.

Proposition 10.9 There exist constants $\bar{\epsilon}>0$ and $E>1$ such that the following properties hold for all nondegenerate open maps $F \in \hat{\mathcal{I}}_{\delta}\left(I^{h} \times I^{v}, \bar{\epsilon}\right)$ : 

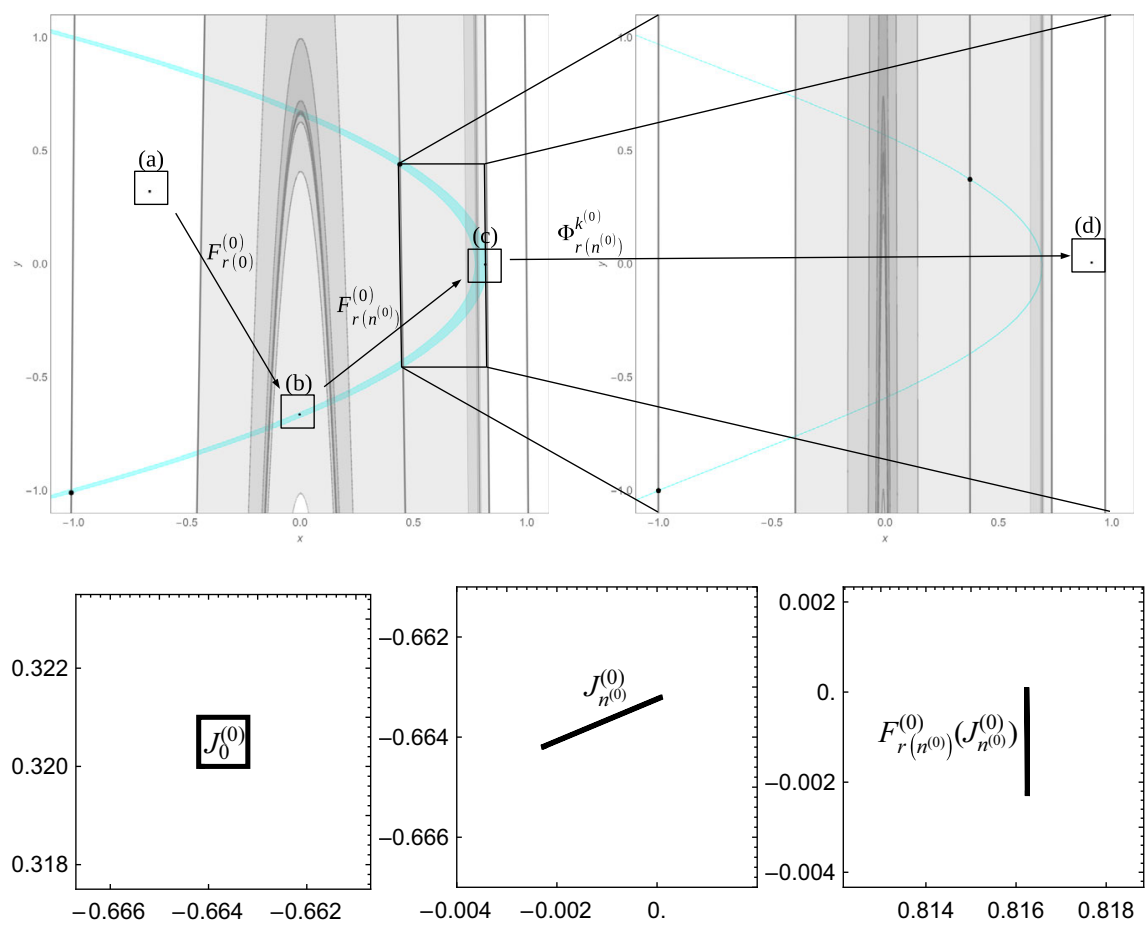

(a) $J_{0}^{(0)}$

(b) $J_{n^{(0)}}^{(0)}$

(c) $F_{n^{(0)}}^{(0)}\left(J_{n^{(0)}}^{(0)}\right)$
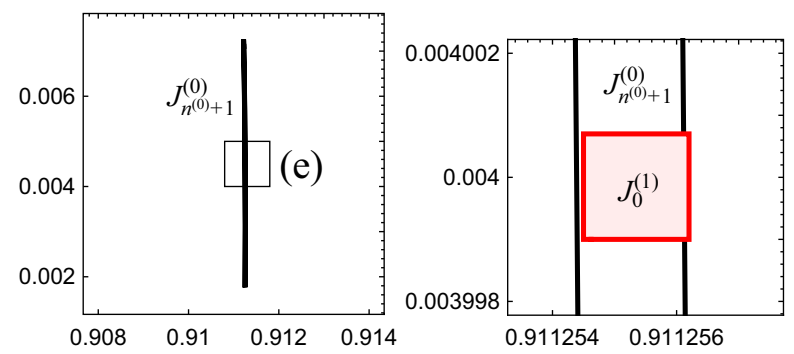

(d) $J_{n^{(0)}+1}^{(0)}$

(e) The first element of a row.

Fig. 12 An example of a double sequence. The graphs are the domains of $F_{0}^{(0)}$ and $F_{1}^{(0)}=F_{0}^{(1)}$. The subfigures $\mathbf{a}-\mathbf{e}$ are elements of the double sequence in an enlarged scale. The scales of $\mathbf{a}-\mathbf{d}$ are the same

Let $J \subset A \cup B$ be a square subset of a wandering domain of $F$ and $\left\{J_{n}^{(j)}\right\}_{n \geq 0,0 \leq j \leq \bar{j}}$ be a $J$-double sequence. Then

1. $\ln l_{0}^{(j+1)} \geq 2 m^{(j)} \ln \left\|\epsilon^{(j)}\right\|+\ln l_{0}^{(j)}$ for all $0 \leq j \leq \bar{j}-1$ and

2. $l_{n+1}^{(j)} \geq E l_{n}^{(j)}$ for all $n<n^{(j)}$ and all $0 \leq j \leq \bar{j}$. 
Proof Let $\bar{\epsilon}>0$ be small enough that Proposition 9.11 and Corollary 10.6 hold.

When the elements stay inside the good regions, the horizontal sizes are determined by the expansion estimate (Proposition 9.11) since the first element of a row is a square. This gives the second inequality.

Let $N=n^{(j)}$. When the element $J_{N}^{(j)}$ enters the bad region, we have $l_{0}^{(j+1)}=w_{N+1}^{(j)}$ by definition. Thus, $l_{0}^{(j+1)}$ can be estimated by the contraction of thickness (Corollary 10.6). That is,

$$
\begin{aligned}
\ln l_{0}^{(j+1)} & =\ln w_{N+1}^{(j)} \geq 2 \sum_{n=0}^{N} \ln \left\|\epsilon_{r(n)}^{(j)}\right\|^{2}+\ln w_{0}^{(j)} \\
& \geq 2(N+1) \ln \left\|\epsilon_{r(N)}^{(j)}\right\|^{2}+\ln l_{0}^{(j)}
\end{aligned}
$$

This proves the first inequality.

The next proposition relates the perturbation $\epsilon$ of two consecutive rows. It shows that the perturbation contracts at an enormous rate because the map is renormalized many times when an element enters the bad region.

Proposition 10.10 There exist constants $\bar{\epsilon}>0$ and $\alpha>0$ such that for all nondegenerate open maps $F \in \hat{\mathcal{I}}_{\delta}\left(I^{h} \times I^{v}, \bar{\epsilon}\right)$, we have

$$
\ln \left\|\epsilon^{(j+1)}\right\| \leq\left\|\epsilon^{(j)}\right\|^{-3 \alpha} \ln \left\|\epsilon^{(j)}\right\|
$$

for all $0 \leq j \leq \bar{j}-1$.

Proof Let $L=k^{(j)}, M=K^{(j)}$, and $N=n^{(j)}$. By Corollary 4.20, we have

$$
\begin{aligned}
\ln \left\|\epsilon^{(j+1)}\right\| & \leq \ln \left\|\epsilon_{0}^{(j+1)}\right\|=\ln \left\|\epsilon_{r(N+1)}^{(j)}\right\| \leq 2^{L}\left[\ln \left\|\epsilon^{(j)}\right\|+\ln c\right] \\
& \leq 2^{L-1} \ln \left\|\epsilon^{(j)}\right\|
\end{aligned}
$$

when $\bar{\epsilon}>0$ is small enough.

Consider the first element in the $j$-th row that enters the bad region. We have $L \equiv k^{(j)}>M \equiv K^{(j)}$. By Proposition 8.2 and the base change formula, we get

$$
2^{L}>2^{M}=\left(\lambda^{M}\right)^{\frac{\ln 2}{\ln \lambda}} \geq c^{\prime}\left\|\epsilon^{(j)}\right\|^{-\frac{\ln 2}{2 \ln \lambda}}
$$

for some constant $c^{\prime}>0$. Let $\alpha=\frac{\ln 2}{8 \ln \lambda}>0$. Combining (10.5) and (10.6), we obtain

$$
\ln \left\|\epsilon^{(j+1)}\right\| \leq \frac{c^{\prime}}{2}\left\|\epsilon^{(j)}\right\|^{-4 \alpha} \ln \left\|\epsilon^{(j)}\right\|<\left\|\epsilon^{(j)}\right\|^{-3 \alpha} \ln \left\|\epsilon^{(j)}\right\|
$$

when $\bar{\epsilon}>0$ is small enough. 


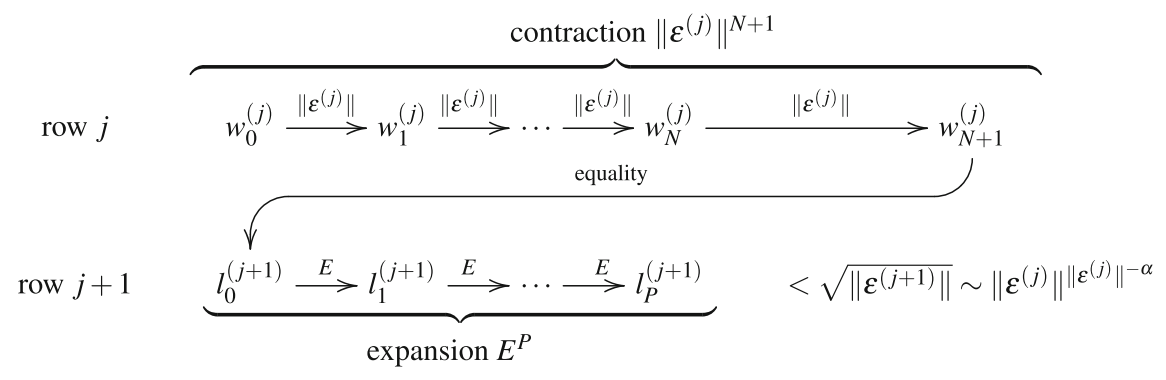

Fig. 13 The contraction, the expansion, and the sizes of the elements in the $j$-th and the $(j+1)$-th rows. Here $N=n^{(j)}$ and $P=n^{(j+1)}$

\subsection{A closest approach can enter bad regions at most finitely many times}

In this subsection, we prove that the number of rows is finite (Proposition 10.13). Even though the horizontal size is contracted when an element in a closest approach enters the bad region, this does not occur often because the size of the bad region is small. In fact, the sizes of the bad regions also contract substantially whenever an element enters a bad region (Proposition 10.10). We will use the Two-Row Lemma (Lemma 10.11) to relate the two types of contraction. Roughly speaking, by applying the Two-Row Lemma inductively (Lemma 10.12), we show that the bad regions contract much faster than the thicknesses. ${ }^{3}$ This implies that a double sequence cannot have infinitely many rows (Proposition 10.13).

We first prove the Two-Row Lemma.

Lemma 10.11 (The Two-Row Lemma) There exist constants $\bar{\epsilon}>0, E>1$, and $\alpha>0$ such that the following property holds for all nondegenerate open maps $F \in \hat{\mathcal{I}}_{\delta}\left(I^{h} \times I^{v}, \bar{\epsilon}\right)$ :

Let $J \subset A \cup B$ be a square subset of a wandering domain of $F$ and $\left\{J_{n}^{(j)}\right\}_{n \geq 0,0 \leq j \leq \bar{j}}$ be a $J$-double sequence. Then

$$
m^{(j)}>\frac{\ln E}{-2 \ln \left\|\epsilon^{(j)}\right\|} m^{(j+1)}+\left(\frac{1}{\left\|\epsilon^{(j)}\right\|}\right)^{2 \alpha}+\frac{1}{-2 \ln \left\|\epsilon^{(j)}\right\|} \ln l_{0}^{(j)}
$$

for all $0 \leq j \leq \bar{j}-2$, where $m^{(j)}=n^{(j)}+1$.

The Two-Row Lemma is the key lemma. It relates the size of the bad region to the contraction and expansion of sizes in two consecutive rows. See Fig. 13

\footnotetext{
3 However, we also need to take the time span in good regions into account because the units of the contraction rates do not match: one is per step but the other is per row. The expansion and the time span in good regions turn out to be crucial and they make the proof nontrivial.
} 
for an illustration. The left-hand side of (10.7) comes from the contraction of thicknesses in the $j$-th row. The right-hand side contains three terms. The first term comes from the expansion of horizontal sizes in the $(j+1)$-th row (see (10.9) in the proof). If the expansion is large, then the contraction in the $j$-th row must be substantial because the size of the element $J_{P}^{(j+1)}$ cannot exceed the size of the bad region where $P=n^{(j+1)}$. The second term comes from the size of the bad region of the $(j+1)$-th row (see (10.8) in the proof). The quantity is large because the size $\left\|\epsilon^{(j+1)}\right\|$ of the bad region of the $(j+1)$ th row is much smaller than the contraction rate $\left\|\epsilon^{(j)}\right\|$ of thicknesses in the $j$-th row (Proposition 10.10). The last term comes from the size of the initial element $J_{0}^{(j)}$.

Proof of Lemma 10.11 First, we consider the $(j+1)$-th row. Let $P=n^{(j+1)}$. The horizontal size of $J_{P}^{(j+1)}$ cannot exceed the size of the bad region. By Proposition 8.2(B2), there exists a constant $c>0$ such that

$$
l_{P}^{(j+1)}<2 c \sqrt{\left\|\epsilon^{(j+1)}\right\|} .
$$

Also, the horizontal sizes in the $(j+1)$-th row expand at a definite rate. There exists a constant $E>1$ such that

$$
E^{P} l_{0}^{(j+1)} \leq l_{P}^{(j+1)}
$$

by Proposition 10.9. After combining (10.8) and (10.9), we get

$$
\ln l_{0}^{(j+1)}<-m^{(j+1)} \ln E+\frac{1}{2} \ln \left\|\epsilon^{(j+1)}\right\|+(\ln E+\ln 2 c) .
$$

Then, we consider the $j$-th row. The thickness of the elements in the $j$-th row contracts. By Proposition 10.9 and (10.10), we have

$$
2 m^{(j)} \ln \left\|\epsilon^{(j)}\right\|<-m^{(j+1)} \ln E+\frac{1}{2} \ln \left\|\epsilon^{(j+1)}\right\|+(\ln E+\ln 2 c)-\ln l_{0}^{(j)} .
$$

Since $\ln \left\|\epsilon^{(j)}\right\|<0$, we deduce that

$$
m^{(j)}>\frac{\ln E}{-2 \ln \left\|\epsilon^{(j)}\right\|} m^{(j+1)}+\left(\frac{1}{4} \frac{\ln \left\|\epsilon^{(j+1)}\right\|}{\ln \left\|\epsilon^{(j)}\right\|}+\frac{\ln E+\ln 2 c}{2 \ln \left\|\epsilon^{(j)}\right\|}\right)+\frac{\ln l_{0}^{(j)}}{-2 \ln \left\|\epsilon^{(j)}\right\|} .
$$

Finally, we apply Proposition 10.10 to simplify the second term. We get 


$$
\begin{aligned}
m^{(j)}> & \frac{\ln E}{-2 \ln \left\|\epsilon^{(j)}\right\|} m^{(j+1)} \\
& +\left(\frac{1}{\left\|\epsilon^{(j)}\right\|}\right)^{2 \alpha}\left[\frac{1}{4}\left(\frac{1}{\left\|\epsilon^{(j)}\right\|}\right)^{\alpha}+\frac{\ln E+\ln 2 c}{2 \ln \left\|\epsilon^{(j)}\right\|}\left\|\epsilon^{(j)}\right\|^{2 \alpha}\right] \\
& +\frac{1}{-2 \ln \left\|\epsilon^{(j)}\right\|} \ln l_{0}^{(j)} \\
> & \frac{\ln E}{-2 \ln \left\|\epsilon^{(j)}\right\|} m^{(j+1)}+\left(\frac{1}{\left\|\epsilon^{(j)}\right\|}\right)^{2 \alpha}+\frac{1}{-2 \ln \left\|\epsilon^{(j)}\right\|} \ln l_{0}^{(j)}
\end{aligned}
$$

when $\bar{\epsilon}$ is sufficiently small.

Next, we use the Two-Row Lemma to prove that a double sequence has at most finitely many rows. Before carrying out a careful proof, we first use a reduced version

$$
m^{(j)}>\frac{1}{-\ln \left\|\epsilon^{(j)}\right\|} m^{(j+1)}+\left(\frac{1}{\left\|\epsilon^{(j)}\right\|}\right)^{\alpha}
$$

of the inequality (10.7) to give an intuition of how the Two-Row Lemma works.

First, we consider the first two rows: the 0 -th and 1-st rows. At this moment, we have no information about $m^{(1)}$. The recurrence relation (10.11) produces a lower bound from only the second term

$$
m^{(0)}>0+\left(\frac{1}{\left\|\epsilon^{(0)}\right\|}\right)^{\alpha} .
$$

The size of the bad region, $\left\|\epsilon^{(1)}\right\|$, is much smaller than the contraction rate of thicknesses, $\left\|\epsilon^{(0)}\right\|$ (Proposition 10.10). To make $J_{n^{(1)}}^{(1)}$ small enough to enter the bad region, $m^{(0)}$ is forced to be large according to the relations in Fig. 13. This yields a large lower bound from the second term.

Then, we add one row into the estimation. Consider the first three rows: the 0 -th, 1-st, and 2-nd rows. We apply the recurrence relation (10.11) to the 1-st and 2-nd rows. For the same reason, we have

$$
m^{(1)}>\left(\frac{1}{\left\|\epsilon^{(1)}\right\|}\right)^{\alpha} \text {. }
$$

After substituting (10.13) into the expansion term of (10.11), we get

$$
m^{(0)}>\underbrace{\frac{1}{-\ln \left\|\epsilon^{(0)}\right\|}\left(\frac{1}{\left\|\epsilon^{(1)}\right\|}\right)^{\alpha}}_{\text {improvement from the 2-nd row }}+\left(\frac{1}{\left\|\epsilon^{(0)}\right\|}\right)^{\alpha} .
$$


Unlike the lower bound (10.12) estimated from only two rows, the 2-nd row improves the lower bound by knowing that $m^{(1)}$ is large. In fact, the improvement (the first term) is much larger than the original estimate (the second term) because of Proposition 10.10.

Then we continue to add more rows. Each time when we include another row, we improve the lower bound of the time span in good regions. By induction, we will show that the lower bound goes to infinity if a double sequence has infinitely many rows. Roughly speaking, the sizes of bad regions contract much faster than the thicknesses. Therefore, a double sequence has at most finitely many rows.

To sum up, the size of the bad region and the contraction of thickness come from the $\epsilon$ of different rows (as illustrated in Fig. 13). The bad region comes from the $(j+1)$-th row but the contraction comes from the $j$-th row. While $\epsilon^{(j+1)}$ is much smaller than $\epsilon^{(j)}$, this forces $n^{(j)}$ to be large (e.g. (10.12) and (10.13)). After applying the large $n^{(j)}$ to the expansion term of (10.7), the huge expansion boosts the time span in good regions significantly by backward induction (e.g. (10.14)). This makes the proof work.

The complete estimate is done by the following lemma.

Lemma 10.12 There exist constants $\bar{\epsilon}>0$ and $\alpha>0$ such that the following property holds for all nondegenerate open maps $F \in \hat{\mathcal{I}}_{\delta}\left(I^{h} \times I^{v}, \bar{\epsilon}\right)$ :

Let $J \subset A \cup B$ be a square subset of a wandering domain of $F$ and $\left\{J_{n}^{(j)}\right\}_{n \geq 0,0 \leq j \leq \bar{j}}$ be a $J$-double sequence. Then

$$
m^{(j)}=n^{(j)}+1>\frac{1}{\left\|\epsilon^{(j)}\right\|^{\alpha}} \frac{1}{\left\|\epsilon^{(j+k)}\right\|^{\alpha}}+\frac{1}{-2 \ln \left\|\epsilon^{(j)}\right\|} \ln l_{0}^{(j)}
$$

for all $j$ and $k$ with $0 \leq j \leq \bar{j}-2$ and $0 \leq k \leq(\bar{j}-2)-j$.

Proof In the lemma, the value $k+2$ is the number of rows that we use to estimate the lower bound. We prove that (10.15) holds for all $0 \leq j \leq \bar{j}-k-2$ by induction on $k \leq \bar{j}-2$. Let $\bar{\epsilon}$ be small enough that Proposition 10.9, Proposition 10.10, and Lemma 10.11 hold.

The base case $k=0$ follows from (10.7).

Assume that there exists $k$ with $1 \leq k \leq \bar{j}-2$ such that (10.15) holds for all $j$ with $0 \leq j \leq \bar{j}-k-2$. Applying the induction hypothesis to (10.7), we get

$$
\begin{aligned}
m^{(j)}> & \frac{\ln E}{-2 \ln \left\|\epsilon^{(j)}\right\|} \frac{1}{\left\|\epsilon^{(j+1)}\right\|^{\alpha}} \frac{1}{\left\|\epsilon^{(j+k+1)}\right\|^{\alpha}}+\frac{\ln E}{-2 \ln \left\|\epsilon^{(j)}\right\|} \frac{1}{-2 \ln \left\|\epsilon^{(j+1)}\right\|} \ln l_{0}^{(j+1)} \\
& +\frac{1}{-2 \ln \left\|\epsilon^{(j)}\right\|} \ln l_{0}^{(j)} .
\end{aligned}
$$


To simplify the first term on the right hand side, we apply the inequality $\ln x<x$ and Proposition 10.10. Then

$$
\frac{\ln E}{-2 \ln \left\|\epsilon^{(j)}\right\|} \frac{1}{\left\|\epsilon^{(j+1)}\right\|^{\alpha}}>\left(\frac{\ln E}{2} \frac{1}{\left\|\epsilon^{(j)}\right\|}\right)\left(\frac{1}{\left\|\epsilon^{(j)}\right\|}\right)^{\alpha\left\|\epsilon^{(j)}\right\|^{-3 \alpha}-2}>\frac{2}{\left\|\epsilon^{(j)}\right\|^{\alpha}}
$$

when $\bar{\epsilon}$ is small.

For the second term on the right hand side, we apply Proposition 10.9:

$$
\begin{aligned}
& \frac{\ln E}{-2 \ln \left\|\epsilon^{(j)}\right\|} \frac{1}{-2 \ln \left\|\epsilon^{(j+1)}\right\|} \ln l_{0}^{(j+1)} \\
& >\frac{\ln E}{2 \ln \left\|\epsilon^{(j+1)}\right\|} m^{(j)}+\frac{\ln E}{-2 \ln \left\|\epsilon^{(j)}\right\|} \frac{1}{-2 \ln \left\|\epsilon^{(j+1)}\right\|} \ln l_{0}^{(j)} .
\end{aligned}
$$

Inserting the results in (10.16), we obtain

$$
\begin{aligned}
m^{(j)} & >\left(1+\frac{\ln E}{-2 \ln \left\|\epsilon^{(j+1)}\right\|}\right)^{-1} \frac{2}{\left\|\epsilon^{(j)}\right\|^{\alpha}} \frac{1}{\left\|\epsilon^{(j+k+1)}\right\|^{\alpha}}+\frac{1}{-2 \ln \left\|\epsilon^{(j)}\right\|} \ln l_{0}^{(j)} \\
& >\frac{1}{\left\|\epsilon^{(j)}\right\|^{\alpha}} \frac{1}{\left\|\epsilon^{(j+k+1)}\right\|^{\alpha}}+\frac{1}{-2 \ln \left\|\epsilon^{(j)}\right\|} \ln l_{0}^{(j)}
\end{aligned}
$$

when $\bar{\epsilon}$ is small. Therefore, the inequality (10.15) also holds for $k+1$ and hence the lemma is proved by induction.

The lemma shows that the size of the bad region from $k+1$ rows ahead gives a significant boost to the lower bound of the time span in good regions. If a double sequence has infinitely many rows, then the lower bound goes to infinity as $k \rightarrow \infty$, which yields a contradiction. This proves the following.

Proposition 10.13 There exists a constant $\bar{\epsilon}>0$ such that for all nondegenerate open maps $F \in \hat{\mathcal{I}}_{\delta}\left(I^{h} \times I^{v}, \bar{\epsilon}\right)$, a double sequence has finite rows.

\subsection{Nonexistence of wandering domains}

Finally, we prove the main theorem.

Theorem 10.14 There exists a constant $\bar{\epsilon}>0$ such that an open map $F \in$ $\mathcal{I}_{\delta}\left(I^{h} \times I^{v}, \bar{\epsilon}\right)$ does not have wandering domains.

Proof We prove the theorem by contradiction. Suppose that $F$ has a wandering domain. By Proposition 4.14 and Corollary 6.4, we may assume without loss of generality that $F \in \hat{\mathcal{I}}_{\delta}\left(I^{h} \times I^{v}, \bar{\epsilon}\right)$ by applying some renormalizations to the map. By iterating the wandering domain and selecting a subset, we can let $J$ be a square subset of a wandering domain in $A(F) \cup B(F)$. 
We may also assume without loss of generality that the $J$-closest approach $\left\{J_{n}\right\}_{n=0}^{\infty}$ stays in the good regions forever by starting from the tail of the rescaled orbit since a closest approach can enter the bad regions at most finitely many times (Proposition 10.13). Thus, we have

$$
\lim _{n \rightarrow \infty} l_{n}=\infty
$$

by Proposition 9.11. This is a contradiction. Therefore, a strongly dissipative infinitely renormalizable Hénon-like map cannot have a wandering domain.

Remark 10.15 The theorem also applies to infinitely CLM-renormalizable maps. This is because, without loss of generality, we can always start from a Hénon-like map that is close to the map $G$ by the hyperbolicity of the renormalization operator, and all maps that are close to $G$ are renormalizable.

As an immediate consequence, we have the following corollary.

Corollary 10.16 There exists a constant $\bar{\epsilon}>0$ such that for any nondegenerate open map $F \in \mathcal{I}_{\delta}\left(I^{h} \times I^{v}, \bar{\epsilon}\right)$, the union of the stable manifolds is dense in the domain.

From the classification of the $\omega$-limit sets $[28,48]$, almost all orbits approach the renormalization Cantor set that is conjugate to the dyadic adding machine. However, the theorem shows that the orbits that do not approach the Cantor set form a dense set in the domain.

Acknowledgements The author thanks Marco Martens for many discussions on Hénon maps, a careful reading of the manuscript, and useful comments on the results. The author thanks Jerzy Trzeciak and the referee for comments that greatly improved the manuscript. The author also thanks Department of Mathematics, Stony Brook University for financial support.

Open Access This article is distributed under the terms of the Creative Commons Attribution 4.0 International License (http://creativecommons.org/licenses/by/4.0/), which permits unrestricted use, distribution, and reproduction in any medium, provided you give appropriate credit to the original author(s) and the source, provide a link to the Creative Commons license, and indicate if changes were made.

\section{References}

1. Arosio, L., Benini, A.M., Fornæss, J.E., Peters, H.: Dynamics of transcendental Hénon maps. Math. Ann. (2018). https://doi.org/10.1007/s00208-018-1643-6

2. Astorg, M., Buff, X., Dujardin, R., Peters, H., Raissy, J.: A two-dimensional polynomial mapping with a wandering Fatou component. Ann. Math. 184, 263-313 (2016). https:// doi.org/10.4007/annals.2016.184.1.2

3. Baker, I.N.: An entire function which has wandering domains. J. Aust. Math. Soc. A 22(02), 173-176 (1976). https://doi.org/10.1017/S1446788700015287

4. Bedford, E.: Dynamics of polynomial diffeomorphisms of $\mathbb{C}^{2}$ : Foliations and laminations. arXiv:1501.01402 (2015) 
5. Blokh, A.M., Lyubich, M.Y.: Non-existence of wandering intervals and structure of topological attractors of one dimensional dynamical systems 2. Ergod. Theor. Dyn. Syst. 9(04), 751-758 (1989). https://doi.org/10.1017/S0143385700005319

6. Bonatti, C., Gambaudo, J.M., Lion, J.M., Tresser, C.: Wandering domains for infinitely renormalizable diffeomorphisms of the disk. Proc. Am. Math. Soc. (1994). https://doi.org/ 10.1090/S0002-9939-1994-1223264-0

7. Brin, M., Stuck, G.: Introduction to Dynamical Systems. Cambridge University Press, Cambridge (2002). https://doi.org/10.1017/CBO9780511755316

8. Campanino, M., Epstein, H., Ruelle, D.: On Feigenbaum's functional equation $g \circ$ $g(\lambda x)+\lambda g(x)=0$. Topology 21(2), 125-129 (1982). https://doi.org/10.1016/00409383(82)90001-5

9. Colli, E., Vargas, E.: Non-trivial wandering domains and homoclinic bifurcations. Ergod. Theory Dyn. Syst. 21(06), 1657-1681 (2001). https://doi.org/10.1017/ S0143385701001791

10. Cremer, H.: Über die schrödersche funktionalgleichung und das schwarzsche eckenabbildungsproblem. Leipziger Berichte 84, 291-324 (1932)

11. de Carvalho, A., Lyubich, M., Martens, M.: Renormalization in the Hénon family, I: universality but non-rigidity. J. Stat. Phys. 121(5-6), 611-669 (2005). https://doi.org/10.1007/ s10955-005-8668-4

12. de Faria, E., de Melo, W., Pinto, A.: Global hyperbolicity of renormalization for $C^{r}$ unimodal mappings. Ann. Math. (2006). https://doi.org/10.4007/annals.2006.164.731

13. de Melo, W.: A finiteness problem for one-dimensional maps. Proc. Am. Math. Soc. 101(4), 721-727 (1987). https://doi.org/10.1090/S0002-9939-1987-0911040-1

14. de Melo, W., Pinto, A.A.: Rigidity of $C^{2}$ infinitely renormalizable unimodal maps. Commun. Math. Phys. 208(1), 91-105 (1999). https://doi.org/10.1007/s002200050749

15. de Melo, W., van Strien, S.: One-dimensional dynamics: the Schwarzian derivative and beyond. Bull. Am. Math. Soc. 18(2), 159-162 (1988). https://doi.org/10.1090/S02730979-1988-15633-9

16. de Melo, W., van Strien, S.: A structure theorem in one dimensional dynamics. Ann. Math. 129(3), 519-546 (1989). https://doi.org/10.2307/1971516

17. de Melo, W., van Strien, S.: One-Dimensional Dynamics. Springer, Berlin (1993). https:// doi.org/10.1007/978-3-642-78043-1

18. Denjoy, A.: Sur les courbes définies par les équations différentielles à la surface du tore. Journal de Mathématiques Pures et Appliquées 11, 333-376 (1932)

19. Dujardin, R.: Hénon-like mappings in $\mathbb{C}^{2}$. Am. J. Math. 126(2), 439-472 (2004). https:// doi.org/10.1353/ajm.2004.0010

20. Epstein, H., Lascoux, J.: Analyticity properties of the Feigenbaum function. Commun. Math. Phys. 81(3), 437-453 (1981). https://doi.org/10.1007/BF01209078

21. Eremenko, A.E., Lyubich, M.Y.: Examples of entire functions with pathological dynamics. J. Lond. Math. Soc. (2) 36(3), 458-468 (1987). https://doi.org/10.1112/jlms/s2-36.3.458

22. Eremenko, A.E., Lyubich, M.Y.: Dynamical properties of some classes of entire functions. Annales de l'institut Fourier 42(4), 989-1020 (1992). https://doi.org/10.5802/aif.1318

23. Fatou, P.: Sur les équations fonctionnelles. Soc. Math. France Bull. 47, 161-271 (1919)

24. Fatou, P.: Sur les équations fonctionnelles. Soc. Math. France Bull. 48, 33-94 (1920)

25. Fatou, P.: Sur les équations fonctionnelles. Soc. Math. France Bull. 48, 208-314 (1920)

26. Fornæss, J.E., Sibony, N.: Fatou and Julia sets for entire mappings in $\mathbb{C}^{k}$. Math. Ann. 311(1), 27-40 (1998). https://doi.org/10.1007/s002080050174

27. Friedland, S., Milnor, J.: Dynamical properties of plane polynomial automorphisms. Ergod. Theory Dyn. Syst. 9(1), 67-99 (1989). https://doi.org/10.1017/S014338570000482X

28. Gambaudo, J.M., van Strien, S., Tresser, C.: Hénon-like maps with strange attractors: there exist $C^{\infty}$ Kupka-Smale diffeomorphisms on $S^{2}$ with neither sinks nor sources. Nonlinearity 2(2), 287 (1989). https://doi.org/10.1088/0951-7715/2/2/005 
29. Goldberg, L.R., Keen, L.: A finiteness theorem for a dynamical class of entire functions. Ergod. Theory Dyn. Syst. 6(02), 183-192 (1986). https://doi.org/10.1017/ S0143385700003394

30. Guckenheimer, J.: Sensitive dependence to initial conditions for one dimensional maps. Commun. Math. Phys. 70(2), 133-160 (1979). https://doi.org/10.1007/BF01982351

31. Hall, G.R.: A $C^{\infty}$ Denjoy counterexample. Ergod. Theory Dyn. Syst. 1(03), 261-272 (1981). https://doi.org/10.1017/S0143385700001243

32. Harrison, J.: Unsmoothable diffeomorphisms. Ann. Math. 102(1), 85-94 (1975). https:// doi.org/10.2307/1970975

33. Harrison, J.: Unsmoothable diffeomorphisms on higher dimensional manifolds. Proc. Am. Math. Soc. 73(2), 249-255 (1979). https://doi.org/10.1090/S0002-9939-1979-0516473-9

34. Hazard, P.: Hénon-like maps with arbitrary stationary combinatorics. Ergod. Theory Dyn. Syst. 31(05), 1391-1443 (2011). https://doi.org/10.1017/S0143385710000398

35. Hazard, P., Martens, M., Tresser, C.: Infinitely many moduli of stability at the dissipative boundary of chaos. Trans. Am. Math. Soc. 370(1), 27-51 (2018). https://doi.org/10.1090/ $\operatorname{tran} / 6940$

36. Hénon, M.: A two-dimensional mapping with a strange attractor. Commun. Math. Phys. 50(1), 69-77 (1976). https://doi.org/10.1007/BF01608556

37. Herman, M.R.: Sur la conjugaison différentiable des difféomorphismes du cercle à des rotations. Publications Mathématiques de l'Institut des Hautes Études Scientifiques 49(1), 5-233 (1979). https://doi.org/10.1007/BF02684798

38. Hu, J., Sullivan, D.P.: Topological conjugacy of circle diffeomorphisms. Ergod. Theory Dyn. Syst. 17(01), 173-186 (1997). https://doi.org/10.1017/s0143385797061002

39. Hubbard, J.H., Oberste-Vorth, R.W.: Hénon mappings in the complex domain. In: Real and Complex Dynamical Systems, pp. 89-132. Springer (1995). https://doi.org/10.1007/97894-015-8439-5_5

40. Hubbard, J.H.: The Hénon mapping in the complex domain. In: Barnsley, M.F., Demko, S.G. (eds.) Chaotic Dynamics and Fractals, pp. 101-111. Academic Press, New York (1986). https://doi.org/10.1016/B978-0-12-079060-9.50010-2

41. Kiriki, S., Soma, T.: Existence of generic cubic homoclinic tangencies for Hénon maps. Ergod. Theory Dyn. Syst. 33(4), 1029-1051 (2013). https://doi.org/10.1017/ S0143385712000168

42. Kiriki, S., Soma, T.: Takens' last problem and existence of non-trivial wandering domains. Adv. Math. 306, 524-588 (2017). https://doi.org/10.1016/j.aim.2016.10.019

43. Kiriki, S., Li, M.C., Soma, T.: Coexistence of invariant sets with and without SRB measures in Hénon family. Nonlinearity 23(9), 2253 (2010). https://doi.org/10.1088/0951-7715/23/ 9/010

44. Kiriki, S., Nakano, Y., Soma, T.: Non-trivial wandering domains for heterodimensional cycles. Nonlinearity 30(8), 3255 (2017). https://doi.org/10.1088/1361-6544/aa7cc6

45. Kwakkel, F.H.: Surface homeomorphisms: the interplay between topology, geometry and dynamics. Phd, University of Warwick (2009). http://webcat.warwick.ac.uk/record= b2317854 S15

46. Kwakkel, F., Markovic, V.: Topological entropy and diffeomorphisms of surfaces with wandering domains. Ann. Acad. Sci. Fenn. Math. 35(2), 503-513 (2010). https://doi.org/ 10.5186/aasfm.2010.3531

47. Lyubich, M.: Non-existence of wandering intervals and structure of topological attractors of one dimensional dynamical systems: 1 . The case of negative Schwarzian derivative. Ergod. Theory Dyn. Syst. 9(04), 737-749 (1989). https://doi.org/10.1017/S0143385700005307

48. Lyubich, M., Martens, M.: Renormalization in the Hénon family, II: the heteroclinic web. Invent. Math. 186(1), 115-189 (2011). https://doi.org/10.1007/s00222-011-0316-9

49. MacKay, R.S., Tresser, C.: Boundary of topological chaos for bimodal maps of the interval. J. Lond. Math. Soc. 2(1), 164-181 (1988). https://doi.org/10.1112/jlms/s2-37.121.164 
50. Martens, M., Winckler, B.: Instability of renormalization. arXiv:1609.04473 (2016)

51. Martens, M., Winckler, B.: On the hyperbolicity of Lorenz renormalization. Commun. Math. Phys. 325(1), 185-257 (2014). https://doi.org/10.1007/s00220-013-1858-z

52. Martens, M., de Melo, W., van Strien, S.: Julia-Fatou-Sullivan theory for real onedimensional dynamics. Acta Math. 168(1), 273-318 (1992). https://doi.org/10.1007/ BF02392981

53. McSwiggen, P.D.: Diffeomorphisms of the torus with wandering domains. Proc. Am. Math. Soc. 117(4), 1175-1186 (1993). https://doi.org/10.1090/S0002-9939-1993-1154247-6

54. McSwiggen, P.D.: Diffeomorphisms of the k-torus with wandering domains. Ergod. Theory Dyn. Syst. 15(06), 1189-1205 (1995). https://doi.org/10.1017/S014338570000986X

55. Milnor, J., Thurston, W.: On iterated maps of the interval. In: Dynamical systems, pp. 465-563. Springer (1988). https://doi.org/10.1007/BFb0082847

56. Milnor, J.: Dynamics in One Complex Variable, 3rd edn. Princeton University Press, Princeton (2011). https://doi.org/10.2307/j.ctt7rnxn

57. Navas, A.: Wandering domains for diffeomorphisms of the k-torus: a remark on a theorem by Norton and Sullivan. arXiv:1702.02251 (2017)

58. Norton, A.: An area approach to wandering domains for smooth surface endomorphisms. Ergod. Theory Dyn. Syst. 11(01), 181-187 (1991). https://doi.org/10.1017/ S0143385700006064

59. Norton, A.: Denjoy's theorem with exponents. Proc. Am. Math. Soc. 127(10), 3111-3118 (1999). https://doi.org/10.1090/S0002-9939-99-04852-2

60. Norton, A., Sullivan, D.: Wandering domains and invariant conformal structures for mappings of the 2-torus. Ann. Acad. Sci. Fenn. Math 21(1), 51-68 (1996)

61. Poincaré, H.: Mémoire sur les courbes définies par une équation différentielle (i,ii,iii,iv). Journal de Mathématiques Pures et Appliquées $(1881,82,85,86)$

62. Raissy, J.: Polynomial skew-products in dimension 2: bulging and wandering Fatou components. Bollettino dell'Unione Matematica Italiana (2016). https://doi.org/10.1007/s40574016-0101-1

63. Schwartz, A.J.: A generalization of a Poincaré-Bendixson theorem to closed twodimensional manifolds. Am. J. Math. (1963). https://doi.org/10.2307/2373135

64. Sharkovskii, A.N., Ivanov, A.F.: $C^{\infty}$-mappings of an interval with attracting cycles of arbitrarily large periods. Ukr. Math. J. 35(4), 455-458 (1983). https://doi.org/10.1007/ BF01093104

65. Sullivan, D.: Quasiconformal homeomorphisms and dynamics I. Solution of the Fatou-Julia problem on wandering domains. Ann. Math. 122(2), 401-418 (1985). https://doi.org/10. 2307/1971308

66. van Strien, S.: One-dimensional dynamics in the new millennium. Discrete Contin. Dyn. Syst. 27(2), 557-588 (2010). https://doi.org/10.3934/dcds.2010.27.557

67. Yoccoz, J.C.: Sur la disparition de la propriété de Denjoy-Koksma en dimension 2. C. R. Acad. Sci. Paris Sér. A-B 291, A655-A658 (1980)

68. Yoccoz, J.C.: Il n'ya pas de contre-exemple de Denjoy analytique. C. R. Acad. Sci. Paris Sér. I Math. 298(7), 141-144 (1984)

Publisher's Note Springer Nature remains neutral with regard to jurisdictional claims in published maps and institutional affiliations. 